\title{
Jovian Chromophore Characteristics from Multispectral HST Images
}

Paul D. Strycker ${ }^{a}$, Nancy J. Chanover ${ }^{a}$,

Amy A. Simon-Miller ${ }^{b}$, Don Banfield ${ }^{c}$, and Peter J. Gierasch ${ }^{c}$

${ }^{a}$ Department of Astronomy, New Mexico State University, Las Cruces, NM 88003 (U.S.A.)

${ }^{\mathrm{b}}$ NASA Goddard Space Flight Center, Greenbelt, MD 20771 (U.S.A.)

${ }^{\mathrm{c}}$ Department of Astronomy, Cornell University, Ithaca, NY 14853 (U.S.A.)

Copyright (C) 2010 Paul D. Strycker, Nancy J. Chanover, Amy A. Simon-Miller, Don Banfield, and Peter J. Gierasch

Number of pages: 39

Number of tables: 8

Number of figures: 29 


\section{Proposed Running Head:}

Jovian Chromophore Characteristics

Please send Editorial Correspondence to:

Paul D. Strycker

Department of Astronomy

New Mexico State University

P.O. Box 30001, MSC 4500

Las Cruces, NM 88003-8001, USA. .

Email: strycker@nmsu.edu

Phone: (575) 646-4834

Fax: (575) 646-1602 


\section{ABSTRACT}

The chromophores responsible for coloring the jovian atmosphere are embedded within Jupiter's vertical aerosol structure. Sunlight propagates through this vertical distribution of aerosol particles, whose colors are defined by $\varpi_{0}(\lambda)$, and we remotely observe the culmination of the radiative transfer as $I / F(\lambda)$. In this study, we employed a radiative transfer code to retrieve $\varpi_{0}(\lambda)$ for particles in Jupiter's tropospheric haze at seven wavelengths in the near-UV and visible regimes. The data consisted of images of the 2008 passage of Oval BA to the south of the Great Red Spot obtained by the Wide Field Planetary Camera 2 on-board the Hubble Space Telescope. We present derived particle colors for locations that were selected from 14 weather regions, which spanned a large range of observed colors. All $\varpi_{0}(\lambda)$ curves were absorbing in the blue, and $\varpi_{0}(\lambda)$ increased monotonically to approximately unity as wavelength increased. We found accurate fits to all $\varpi_{0}(\lambda)$ curves using an empirically derived functional form: $\varpi_{0}(\lambda)=1-A \exp (-B \lambda)$. The best-fit parameters for the mean $\varpi_{0}(\lambda)$ curve were $A=25.4$ and $B=0.0149$ for $\lambda$ in units of nm. We performed a principal component analysis (PCA) on our $\varpi_{0}(\lambda)$ results and found that one or two independent chromophores were sufficient to produce the variations in $\varpi_{0}(\lambda)$. A PCA of $I / F(\lambda)$ for the same jovian locations resulted in principal components (PCs) with roughly the same variances as the $\varpi_{0}(\lambda)$ PCA, but they did not result in a one-to-one mapping of $\mathrm{PC}$ amplitudes between the $\varpi_{0}(\lambda) \mathrm{PCA}$ and $I / F(\lambda)$ PCA. We suggest that statistical analyses performed on $I / F(\lambda)$ image cubes have limited applicability to the characterization of chromophores in the jovian atmosphere due to the sensitivity of $I / F(\lambda)$ to horizontal variations in the vertical aerosol distribution. 
Keywords: JUPITER; JUPITER, ATMOSPHERE; ATMOSPHERES, COMPOSITION; ATMOSPHERES, STRUCTURE 


\section{Background}

\subsection{Chromophores and Two Ways to Define Color}

The jovian atmosphere displays significant contrast in its visible coloration. One or more coloring agent, or chromophore, is necessary to account for the observed color variations between weather regions such as the belts, zones, and vortices. However, the chemical identity, horizontal (latitudinal and longitudinal) and vertical distribution, and number of chromophores are still unknown (West et al., 2004).

When approaching the chromophore problem, we must make a distinction between observed color and particle color. Observed color, $I / F(\lambda)$, is the shape of a reflectance spectrum, which is a culmination of many wavelength-dependent radiative processes in the observed body. Particle color, $\varpi_{0}(\lambda)$, is the shape of the single scattering albedo spectrum, which is a fundamental property of the individual particles.

The relationship between observed colors and particle colors in the jovian atmosphere is not necessarily a one-to-one function. Observed color is equivalent to particle color only in the case wherein isolated chromophores are observed directly, i.e., without any radiative contributions from intervening or adjacent material and without multiple scattering within the chromophore layer. These conditions clearly do not exist in planetary atmospheres: observed $I / F(\lambda)$ is the end result of radiative processes occurring within an atmosphere over distances of tens to hundreds of kilometers that span large variations of pressure, temperature, aerosol abundance, and chemical composition. Thus, $\varpi_{0}(\lambda)$ must 
be derived through radiative transfer modeling of the vertical structure.

\subsection{Chromophore Characteristics via $I / F(\lambda)$}

In order to spectrally characterize Jupiter's atmosphere, most previous studies have analyzed the horizontal variations of $I / F(\lambda)$ (e.g. Owen and Terrile, 1981;

Beebe and Hockey, 1986; West et al., 1986; Thompson, 1990; Dyudina et al., 2001; Simon-Miller et al., 2001a). The main contribution of $I / F(\lambda)$ studies to the chromophore problem is an analysis of the directly observable quantities$I / F(\lambda)$ as a function of latitude, longitude, and viewing geometry-for which any hypothesized distribution of chromophore candidates must be able to account. Although such studies describe real spectral and spatial distributions of observed colors, we cannot assume that the spectral and spatial distributions of the particle colors are equivalent. To some extent, the $I / F(\lambda)$ variations that we study are physically caused by horizontal variations in the atmosphere, such as cloud heights, optical depths, particle radii, etc. These factors may cause much larger variations in $I / F(\lambda)$ than are caused by variations in $\varpi_{0}(\lambda)$.

For example, Thompson (1990) used a cluster analysis to find classifications of locations based on color and albedo. A cluster analysis creates classifications of spectra such that the spectra within a group are mutually similar and the spectral differences between groups are as large as possible. Each spectrum in the data set is assigned to a single group. Thompson (1990) analyzed $0.5^{\circ} \times 0.5^{\circ}$ resolution maps from Voyager 2 acquired in three wideband filters centered at 431, 564, and $599 \mathrm{~nm}$ and one narrowband filter centered at $621 \mathrm{~nm}$, which spanned a weak $\mathrm{CH}_{4}$ absorption band at $619 \mathrm{~nm}$. They found "20 distinct and five tentative units" for classification. While it is true that different com- 
binations of vertical aerosol structure, viewing geometry, and chromophores resulted in the spectral differences defined by their classification scheme, the inclusion of a $\mathrm{CH}_{4}$ absorption band certainly increased their sensitivity to vertical aerosol structure and viewing geometry at the expense of sensitivity to chromophores. Therefore, their classification units are not directly applicable to the number or distribution of chromophores.

Even if the method is an indirect one, an important product of $I / F(\lambda)$ analyses is an estimate of the number of chromophores that are necessary to reproduce the observed variations in $I / F(\lambda)$. Strictly speaking, these estimates are neither an upper nor lower limit to the actual number of unique chromophores that exist in the jovian atmosphere. Unless all other factors that are relevant to radiative transfer are ruled out, it is possible that only one chromophore is necessary. Alternatively, more than one chromophore may be present such that they always coexist spatially with the same mixing ratio and are, therefore, indistinguishable.

A statistical method that has been used to find the number of independent variables contributing to a data set is principal component analysis (PCA) (for formalism, see Preisendorfer and Mobley, 1988; Murtagh and Heck, 1987). Unlike cluster analysis, PCA does not separate spectra into discrete groups. For data containing many spectra, PCA finds (1) a set of orthogonal spectral shapes $^{1}$, which are called principal components (PCs), and (2) the amplitude of each $\mathrm{PC}$ that is necessary to reproduce each spectrum (typically the mean1 We used the term spectral shape here (and hereafter, when referring to results from PCA) because PCs contain both positive and negative coefficients, which cannot be physically interpreted as actual spectra. In a given $\mathrm{PC}$, wavelengths with coefficients of the same sign are correlated and those with opposite signs are anti-correlated. 
subtracted spectrum) in the data by a linear superposition of all PCs. This is a factorization that can be represented as:

$$
V_{i \lambda}=(W H)_{i \lambda}=\sum_{a=1}^{r} W_{i a} H_{a \lambda}
$$

where $V$ is an $m \times r$ matrix that contains $m$ spectra with $r$ wavelengths, $i$ denotes an individual pixel, $\lambda$ denotes an individual wavelength, and $a$ denotes an individual PC. The PCs are chosen so that the first PC is in the "direction" (in $r$-dimensional wavelength space) containing the greatest amount of the spectral variance in the data. All variance in the direction of the first PC is then removed from the data. The second PC lies in the direction of the largest spectral variance that remains, which will necessarily be orthogonal to the direction of the first PC. This process continues until the number of PCs equals $r$, at which point the PCs constitute a complete set of basis vectors for the $r$-dimensional wavelength space.

The advantage of PCA over cluster analysis in determining the number of chromophores is that one can estimate the number of unique spectral shapes that contribute to the total variance of the data. This is accomplished by estimating how many PCs describe real signal in the data and not merely noise. Each PC contains roughly the same amount of uncorrelated noise. Because each successive $\mathrm{PC}$ accounts for less of the total variance, it contains a smaller ratio of (correlated) signal to (uncorrelated) noise. PCs that have a higher order than the number of statistically independent physical processes that are affecting the shapes of the spectra will describe only noise and will all contain approximately equal amounts of the total variance.

There is a noteworthy deficiency in all PCA results. We mentioned above that 
the spectral shape of each successive PC is constrained to be orthogonal to all PCs that account for a larger amount of the variance. Thus, the spectral shapes of PCs 2-r are not accurate representations of the true shapes of spectral variations in the data.

Dyudina et al. (2001) used PCA to spectrally characterize Jupiter's clouds based on Galileo Solid State Imager (SSI) and Near Infrared Mapping Spectrometer (NIMS) data at 26 wavelengths. With their substantial wavelength coverage, inclusion of moderate and strong methane $\left(\mathrm{CH}_{4}\right)$ absorption bands, and inclusion of thermal emission at $5 \mu \mathrm{m}$, they found that $91 \%$ of the spectral variance was contained in the first three PCs. They found one PC that was associated with a violet-absorbing chromophore in a separate PCA of the Great Red Spot (GRS) and its immediate surroundings, but this was the only location in their data that yielded a PC with an anti-correlation between violet reflectivity and all other non-thermal bands. However, only one of their wavelengths $(410 \mathrm{~nm})$ was in the spectral range of the broad chromophore absorption blueward of $\sim 600 \mathrm{~nm}$ (see Fig. 1), and their PCA results were mostly indicative of spatial variations in vertical aerosol structure, which is consistent with their interpretations of them.

Simon-Miller et al. (2001a) conducted a PCA study that was focused on chromophore absorption. Their data consisted of $I / F$-calibrated global maps of Jupiter from HST images acquired in October 1995 and October 1996. The first set contained continuum filters F410M, F555W, and F953N, and the second set added the F673N filter. They determined that only three spectral components are required to explain the deviations from the mean $I / F(\lambda)$. PC1, containing 91\% of the variance, was spectrally gray, and therefore did not correspond to a chromophore. They concluded that PC1 "is probably re- 
lated to density but is not exactly cloud optical depth". PC2 contained $8 \%$ of the variance and indicated the presence of a red chromophore. PC3, presumably arising from the presence of a second chromophore, contained $1 \%$ of the variance and was present in the GRS and some smaller anticyclonic ovals. The 1996 data set with four filters yielded a fourth $\mathrm{PC}$ with a variance $\ll 0.1 \%$, which was less than the observational error.

Thus, Simon-Miller et al. (2001a) constrained the number of statistically independent factors affecting their filter set to three, attributed two of the three to chromophores, obtained a map of each PC's horizontal distribution, and provided a low-resolution spectral shape-though affected by the orthogonality constraint-for the most prominent $I / F(\lambda)$ color variation due to a chromophore (PC2). But to conclusively identify a chromophore, we need to do more than isolate differences in observed color through analyses of $I / F(\lambda)$. We must eventually have a spectrum of the particle color itself.

\subsection{Chromophore Characteristics via $\varpi_{0}(\lambda)$}

Retrievals of $\varpi_{0}(\lambda)$ must be made in the context of an atmospheric model. The complications and degeneracies inherent in radiative transfer models explain why fewer studies have set out to characterize $\varpi_{0}(\lambda)$ than $I / F(\lambda)$. The goal of many radiative transfer studies is to explore the vertical distribution of aerosols, and their data do not contain the appropriate spectral coverage to study $\varpi_{0}(\lambda)$. It is often impractical to obtain center-to-limb feature tracks in several-preferably narrowband-continuum filters in addition to the $\mathrm{CH}_{4}$ absorption band filters that are required for vertical discrimination. 
Simon-Miller et al. (2001b) studied the vertical aerosol structure and particle absorption in the continuum with a radiative transfer analysis of Galileo SSI data. $410 \mathrm{~nm}$ and $756 \mathrm{~nm}$ were the only continuum wavelength for which they had data. They fixed $\varpi_{0}(756)=1$ and explored the range of $\varpi_{0}(410)$ not the number of chromophores nor their spectra. They preformed retrievals of vertical aerosol structure and $\varpi_{0}(410)$ for zones, belts, vortices, and a hot spot. As in the Banfield et al. (1998b) study, which Simon-Miller et al. (2001b) extended by considering particle color, the vertical structure that Simon-Miller et al. (2001b) employed contained as few layers as possible to fit their data. For the majority of locations, their data were "well fit with a three-layer model that contains a thin stratospheric haze, a denser tropospheric haze, and a tropospheric cloud sheet at pressures consistent with an ammonia cloud." The vertical distribution of their $\varpi_{0}(410)$ retrievals showed the chromophores to reside mostly in the tropospheric haze.

Pérez-Hoyos et al. (2009) presented a detailed radiative transfer study of Oval BA and its immediate surroundings to investigate its change in color from white to red (Simon-Miller et al., 2006). They conducted a radiative transfer analysis of HST images acquired before (2005) and after (2006) the color change, retrieving $\varpi_{0}(\lambda)$ in six filters: F250W, F330W, F435W, F550M, F658N, and F892N. They also used a three layer model and found the chromophores to primarily reside in the tropospheric haze, and we adopted this hypothesis for the chromophore location in this work. Their $\varpi_{0}(\lambda)$ retrievals for the Oval BA region are the most complete spectra of jovian chromophores of which we are aware. However, they are not necessarily descriptive of the global chromophore population because it was retrieved from an isolated region of the planet. 
Our goal in this work is to use sufficient spatial and spectral coverage to characterize the particle colors of the global chromophore population. We retrieved $\varpi_{0}(\lambda)$ from multispectral HST WFPC2 images and statistically analyzed the horizontal variations. Section 2 describes the observations, data reduction, and data selection for modeling. Section 3 presents the radiative transfer code, the modeling procedure, and an estimate of the expected degeneracy. Section 4 presents the retrievals and an analysis of the particle color variations. Section 5 discusses the derived chromophore characteristics and explores the extent to which an $I / F(\lambda)$ analysis reaches similar results. Section 6 contains the conclusions.

\section{Observations}

\subsection{Overview}

HST WFPC2 observations of Jupiter were made on 15 May, 28 June, and 8 July 2008, covering a passage of Oval BA south of the Great Red Spot (GRS). Two separate epochs of data exist for 15 May due to the separation between transit times for the GRS and Oval BA. Nine filters sampled the continuum (Fig. 1), and seven of these were narrowband filters, making this data set ideal for color studies at high spatial resolution.

We created a spectral image cube for each epoch of observation. These cubes contain two spatial dimensions (planetographic latitude and System III longitude) and one spectral dimension. Associated with each mapped image in each cube are maps of the three relevant viewing geometry quantities: $\mu, \mu_{0}$, and $\phi$, where $\mu_{0}$ is the cosine of the incidence angle, $\mu$ is the cosine of the 
emission angle, and $\phi$ is the phase angle.

Each spectral image cube contains at least one image per filter. In the cases where two images exist for one filter, both images with their unique viewing geometry data were used. Two F410M images exist for both epochs on 15 May, and two F673N images exist in all epochs. Table 1 contains a summary of the observations and the spectral image cube assignments.

\subsection{Absolute Photometric Calibration, Navigation, and Mapping}

We converted images to $I / F$ using the PHOTFLAM photometry keyword provided by the Space Telescope Science Institute for each filter, the solar flux across each pass band, distance of the Sun to Jupiter, image integration time and pixel solid angle, as in Simon-Miller and Gierasch (2010). In addition to the standard conversion, we corrected the F255W filter throughput for longterm degradation following Gonzaga et al. (2006). After conversion to $I / F$, a comparison of the approximately full disk $I / F(\lambda)$ to a standard Jupiter fulldisk spectrum (Karkoschka, 1998) showed that the F343N filter brightness values appeared anomalously low. This was subsequently confirmed via standard star monitoring, and a new filter throughput was calculated, changing the final $I / F$ by nearly a factor of two (see Gonzaga and Biretta, 2009). With this new value, the final full-disk $I / F(\lambda)$ is consistent with Karkoschka (1998) (Fig. 1).

To properly determine planetary coordinates for mapping, we navigated each image. The navigation was performed using the known sub-spacecraft and sub-solar points, the camera's plate scale and focal length, and the distance to 
Jupiter to size an ellipse with the correct aspect ratio and terminator and limb orientations. The north pole angle was based on Jupiter's position angle on the sky and approximate roll angle of the camera. An initial location was chosen for the ellipse center by manually matching the limb and terminator locations, usually to within a few pixels. An automated routine then located actual limb and terminator points by contrast with the sky background, searching within 20 pixels of an initially estimated ellipse location. The planet center and north angle were then iterated using a least squares minimization until residuals were less than 0.1 pixels, and usually less than 0.05 pixels for most wavelengths.

However, the precision of the navigation was better than the accuracy, which was revealed after we reprojected the images onto cylindrical maps using $\mathrm{MaRC}^{2}$. This software package enabled us to perform reprojections of $I / F, \mu, \mu_{0}$, and $\phi$ at each pixel in the HST images based on Jupiter's oblate spheroidal shape and viewing geometry. With the cylindrical maps we created spectral image cubes for each epoch (Fig. 2) and visually inspected the relative spatial positions of all features across all maps in each cube. Although we attempted to solve minor registration issues using new planet navigations, this procedure did not yield satisfactory results. We found it necessary to manually apply a small shift $\left(\leq 0.29^{\circ}\right.$ planetographic latitude, $\leq 1.43^{\circ}$ longitude) to individual maps in order to have the same latitude and longitude position for a given atmospheric feature in all maps within a given spectral image cube. The maximum displacement of features due to zonal flow during the time elapsed between the first and last images in each image cube was on the order of one

$\overline{2}$ MaRC (Map Reprojections and Conversions) is a free $\mathrm{C}++$ library and program developed by Ossama Othman to produce map projections. Further information and software can be found at http://sourceforge.net/projects/marc/ . 
pixel. We therefore did not attempt to correct for this effect.

\subsection{Data Selection}

Our goal in this work was to demonstrate the possible range of jovian chromophores' particle colors. To achieve this, we needed to retrieve $\varpi_{0}(\lambda)$ from jovian locations with significantly different $I / F(\lambda)$. As the data did not contain feature tracks from center to limb at any wavelength, we could not retrieve $\varpi_{0}(\lambda)$ for individual pixels. Rather, it was necessary to identify groups of pixels to model simultaneously (hereafter, data cuts) in order to improve the range of $\mu$ and $\mu_{0}$-thereby improving our confidence in the model retrievals - as much as possible. Each data cut is extracted from a set of locations that we expect to have similar vertical structures based on spectral similarity. It is not possible to construct a data cut for which it is known a priori that a single vertical structure is contributing to $I / F(\lambda)$. However, we do know a priori that a given vertical structure will produce identical $I / F(\lambda)$ at two locations as $\delta \mu, \delta \mu_{0}$, and $\delta \phi$ approach zero. The $I / F(\lambda)$ of a given vertical structure should have smooth center-to-limb variations because in this case the $I / F(\lambda)$ will only change as a function of the viewing geometry. Therefore, locations with vertical structures that are different from that of a fiducial location can be excluded (to first order) from a data cut by selecting only spectrally similar locations.

To create a data cut, we first selected a fiducial pixel on the mapped data cube and included all pixels within $\pm 0.39^{\circ}$ latitude ( \pm 5 pixels in the mapped images) with similar spectra (for an example, see Fig. 3). The criterion for the 
spectral similarity of a test pixel with the fiducial pixel was:

$$
\frac{\left|I / F_{0, \text { fiducial }}(\lambda)-I / F_{0, \text { test }}(\lambda)\right|}{I / F_{0, \text { fiducial }}(\lambda)}<0.05
$$

for all values of $\lambda$, where:

$$
I / F_{0} \equiv \frac{I / F}{\mu_{0}} .
$$

Thus, the reflectivity of all pixels in a data cut were within $5 \%$ of the fiducial pixel's reflectivity. The selected pixels were split into bins $1^{\circ}$ wide in longitude. We averaged the $I / F, \mu, \mu_{0}$, and $\phi$ for the pixels in each bin in each image of the data cube.

The quantity $I / F_{0}$ was used to compare pixels because this eliminated spectral differences due solely to variable illumination. Doing so assumed a value of $k=1$ in the Minnaert function for limb darkening:

$$
A=A_{M} \mu_{0}^{k} \mu^{k-1}
$$

where $A$ is albedo and $A_{M}$ is the Minnaert albedo. However, we cannot ascertain the true value of $k$ for a given location because of the lack of multiple images of the same feature as its viewing geometry changed. If our assumption that $k=1$ is significantly in error, then there are two consequences for the data selection as the $\mu$ and $\mu_{0}$ distances from the fiducial point increase: (1) pixels with identical vertical structure to the fiducial point may be wrongly excluded in the data cut because their $I / F_{0}(\lambda)$ now differs by $>5 \%$, and (2) pixels with different vertical structures from the fiducial point may be wrongly included in the data cut.

Based on visual inspection of the data cuts produced by this method, it ap- 
peared to satisfactorily select a uniform set locations similar to the fiducial point and reject locations with obviously different clouds. In this sense, it is an improvement upon the practice of including (in one data cut) all longitudes within a narrow latitude range despite the visible heterogeneity that exists in the jovian atmosphere. Our method did, however, appear to prevent our data cuts from including longitudes as close to the limb of the disk as would have otherwise been possible. But, this is of secondary importance compared with successfully excluding pixels that do not belong to the same spectral class as the fiducial point, which we believe that our method reasonably attains.

We selected the fiducial pixels for data cuts from 14 jovian weather regions (Table 2). We did not perform any spectral analysis to determine which fiducial points to select. We visually identified zonal bands with smooth brightness variations in longitude and selected fiducial points manually from them. We did not include highly inhomogeneous bands, such as the South Equatorial Belt, or bands dominated by vortices, such as the southern zones. We also visually identified the vortices and discretely colored regions within the GRS and BA to manually select fiducial points. The spatial coverage of our data cuts is globally representative in the sense that zones, belts, and vortices of various colors are included.

Figure 2 displays all locations used in this study marked on an approximate true-color composite of the data, and Figure 4 shows the average reflectance for all data cuts. Neither the modeling nor subsequent statistical analysis took the regional groupings into account-they are used only as a reference for ease and clarity of discussion. Six zonal regions were selected based on their visible uniformity in longitude, which indicated that many pixels could be grouped into a data cut appropriately: the Equatorial Zone (EZ), the North 
Equatorial Belt (NEB), the North Tropical Zone (NTrZ), the southern area of the North Temperate Belt (NTBs), the northern area of the North Temperate Belt (NTBn), and the North Temperate Zone (NTZ). We also selected eight smaller-scale systems based on their spectral uniqueness: the red annulus, bright annulus, and red center of the GRS; the red annulus and bright center of Oval BA; a small red anticyclone that passed through the perimeter of the GRS (Oval 2); white oval A5; and a red oval embedded in the NEB (hereafter, NEB1). Figure 5 shows the average reflectance for all data cuts within each weather region studied. In total, we selected 1345 data cuts from these 14 regions.

As seen in Table 2, we used many more data cuts from the smaller regions than the zonal regions. The reasons for this are derived from the fact that the zonal regions contain many more samples per data cut. The greater the range of $\mu$ and $\mu_{0}$ that a data cut contains, the better the model fits are constrained. For longitudinally restricted weather systems such as the GRS, the data cuts have only a few $\mu$ and $\mu_{0}$ points, and these are closely spaced. For zones and belts, however, we had extensive coverage in $\mu$ and $\mu_{0}$ by including a large range of longitudes. In order to increase our confidence in model retrievals of the small systems, we modeled many more data cuts taken from them.

\section{Radiative Transfer Model}

\subsection{Model Heritage and Methodology}

The radiative transfer code we employed was originally developed by Banfield et al. (1998b) to analyze Galileo SSI data. Their adding and doubling code was 
based on the work of Hansen and Travis (1974). Banfield et al. (1998b) utilized data taken in filters centered at the strong $\mathrm{CH}_{4}$ band at $889 \mathrm{~nm}$, the moderate $\mathrm{CH}_{4}$ band at $727 \mathrm{~nm}$, and the continuum at $756 \mathrm{~nm}$. Images in each filter were selected for three viewing angles in which the SSI target location was near the terminator, nadir, and limb. The range of pressure levels probed by the three filters combined with observations from multiple viewing angles and smallscale spatial variations of $I / F(\lambda)$ within each data cut enabled Banfield et al. (1998b) to retrieve optical depths and vertical positions of aerosol layers.

Simon-Miller et al. (2001b) supplemented the code by including images from the SSI 410-nm filter and solving for $\varpi_{0}$ at $410 \mathrm{~nm}$ in each aerosol layer. Our work extends the Simon-Miller et al. (2001b) model for use with HST WFPC2 filters and an arbitrary number of images in each filter. For our study, we used Rayleigh gas that contained $\mathrm{H}_{2}, \mathrm{He}$, and $\mathrm{CH}_{4}$ with number mixing ratios with respect to $\mathrm{H}_{2}$ of 0.157 for $\mathrm{He}$ (von Zahn et al., 1998) and 0.00237 for $\mathrm{CH}_{4}$ (Wong et al., 2004). Following Banfield et al. (1998b), we assumed that the aerosols are spherical particles, thereby using Mie scattering theory to determine extinction efficiencies and phase functions. We assumed the aerosol composition to be ammonia ice with an index of refraction of 1.4 (Martonchik et al., 1984). We employed a gamma distribution of particle radii (Mishchenko et al., 1999):

$$
n(r)=c \times r^{(1-3 b) / b} \exp \left(-\frac{r}{r_{0} b}\right), b \in(0,0.5)
$$

where $n$ is the number of particles with radius $r, r_{0}$ is the typical particle radius, $b$ is a characteristic width set to 0.1 , and $c$ is set such that $\int_{r_{\min }}^{r_{\max }} n(r)=1$. For the particle phase function, we used a Henyey-Greenstein phase function with a single asymmetry parameter fit to the Mie phase function for a given 
particle size distribution. We parametrized each aerosol layer by its base pressure, optical depth, and typical particle radius.

There are three aerosol layers and twelve model parameters per layer in our code: base pressure $(P)$, optical depth $(\tau)$ at $502 \mathrm{~nm}$, particle radius $\left(r_{0}\right)$, and single scattering albedo $\left(\varpi_{0}\right)$ for each of nine filters. Table 3 contains gas absorption parameters and Rayleigh scattering parameters. We calculated gas absorption by averaging the $\mathrm{CH}_{4}$ absorption coefficients, $k$, from Karkoschka (1998) over the total system response curve of each filter. We fit both gas absorption and Rayleigh scattering with a single-parameter Beer's Law.

We began this work before Karkoschka and Tomasko (2010) published an updated model of $\mathrm{CH}_{4} k$-values. However, the differences in the $k$-values between the two models were negligible for our filters. The $k$-values reported are so low that Rayleigh scattering entirely dominates gas absorption in the radiative transfer, and we continued with the $k$-values of Karkoschka (1998) due to its simpler treatment. The relative importance of $\mathrm{CH}_{4}$ absorption and Rayleigh scattering is illustrated in Fig. 6, which shows the two-way transmissivity at each wavelength due to $\mathrm{CH}_{4}$ absorption and Rayleigh scattering in a clear, aerosol-free atmosphere. Two-way transmissivity is the fraction of light that remains in the beam-i.e. the fraction that is neither absorbed nor scattered out of the line of sight - for a path from space down to a given pressure and back. The two-way transmissivity curves indicate the vertical sensitivity of the filters. The vertical sensitivity for this set of filters due to $\mathrm{CH}_{4}$ absorption is limited to pressures $\gtrsim 10$ bar.

We estimated the uncertainty in relative photometry $(\sigma)$ in the $I / F$-calibrated images by comparing the standard deviation of pixel values within each bin 
across the weather regions. This is used to calculate the value of $\chi^{2}$ for each model:

$$
\chi^{2}=\frac{1}{n_{o b s}-n_{\text {par }}} \sum_{i=1}^{n_{o b s}} \frac{\left(y_{i}-f_{i}\right)^{2}}{\sigma^{2}}
$$

where $n_{o b s}$ is the number of observations, $n_{p a r}$ is the number of free parameters, $y_{i}$ is the observed $I / F$ at the viewing geometry and wavelength of observation $i, f_{i}$ is the corresponding model result, and $\sigma$ is the standard deviation of the filter used for observation $i$.

\subsection{Vertical Structure and Parameter Space}

Figure 7 shows the format for the vertical structure used in our models. We included three adjoining aerosol layers to model pressures $\leq 1$ bar, similar to the standard model presented in West et al. (2004) (see their Fig. 5.15): a stratospheric haze, tropospheric haze, and tropospheric cloud. We use the term "haze" to refer to aerosol layers that contain both Rayleigh-scattering gas and aerosols. We call a layer that solely contains aerosols a "cloud".

The base of the stratospheric haze is attached to the top of the tropospheric haze, and the base of the tropospheric haze is attached to a tropospheric cloud, which is located in the vicinity of the ammonia condensation level. The top and bottom of the cloud are set to the same pressure. The base of the model atmosphere is a semi-infinite Rayleigh-scattering gas layer.

The infinitely-thin tropospheric cloud-which was also used by Banfield et al. (1998b) and Simon-Miller et al. (2001b) - is a numerical convenience and is not necessary to fit the data. It results in an aerosol layer without any Rayleigh- 
scattering gas mixed in, which is a convenient approximation when aerosols dominate the radiative transfer. This approximation becomes increasingly degenerate in pressure as the total $\tau$ of the true cloud decreases and as the vertical extent of the true cloud increases. For an optically thick cloud, the pressure level of the infinitely thin cloud is where the top of the physically extended cloud would be located. For an optically thin cloud, the pressure level of the infinitely thin cloud would probably be near the middle of the physically extended cloud.

We implemented several parameter constraints to avoid fitting degenerate parameter combinations. Table 4 shows all fixed and free model parameters, and Table 5 contains the initial parameter values. Due to the limited vertical discrimination possible with our data, we fixed the pressure parameters. To explore the relevant pressure range of tropospheric clouds, we used three different models with tropospheric cloud pressures at $0.4,0.7$, and 1.0 bar. The interface between the stratospheric haze and tropospheric haze was fixed at the approximate location of the tropopause $(0.1 \mathrm{bar})$ in all models. Although some studies have found that a clearing in the aerosols near the tropopause was necessary to fit their data (Banfield et al., 1998a; de Pater et al., 2010a), we do not believe that we can discern a difference between a gap or no gap in the aerosols with our data due to degeneracies with the pressure parameters $($ see $§ 3.4)$.

We fixed the particle radii at the central values of the ranges reported by Simon-Miller et al. (2001b) after finding very little ability to constrain radii retrievals with our data. We derived $\tau$ for all three layers but constrained them such that $\tau \leq 50$. Without a clear ability to discern the altitude of each aerosol layer, we are not able to speculate in this work about the aspects of 
the vertical structure or changes therein that are associated with chromophore production/visibility. See Pérez-Hoyos et al. (2009) and de Pater et al. (2010b) for discussions of chromophore variability mechanisms.

We fit $\varpi_{0}(255-343 \mathrm{~nm})$ only in the stratospheric haze due to the shallow penetration depth of UV light, and fit $\varpi_{0}(375-673 \mathrm{~mm})$ only in the tropospheric haze. Retrieved $\varpi_{0}(255)$ and $\varpi_{0}(343)$ values are shown in a few instances but are not discussed. They are necessary parameters for the radiative transfer model, but they are not well constrained.

We tied the $\varpi_{0}(255-343 \mathrm{~nm})$ in the tropospheric haze to the value of $\varpi_{0}(375$ $\mathrm{nm})$ for several reasons. The sensitivity of the model to $\varpi_{0}(255-343 \mathrm{~nm})$ in the tropospheric haze was very low. When we left these as free parameters while testing the model, they often converged to values near zero. This increased the values derived for $\varpi_{0}(255-343 \mathrm{~nm})$ in the stratospheric haze, which must be free parameters to properly fit $\tau_{1}$ in the stratosphere. We concluded that a physically reasonable solution was to force $\varpi_{0}(255-375 \mathrm{~nm})$ in the tropospheric haze to have a spectral slope of zero. We assumed conservative scattering $\left(\varpi_{0}=1.0\right)$ for all fixed $\varpi_{0}$ parameters.

Simon-Miller et al. (2001b) determined that Jupiter's $I / F$ at $410 \mathrm{~nm}$ was consistent with models in which chromophores were located only in the tropospheric haze above the main ammonia cloud deck. We restricted the aerosol particles' visible coloration to the tropospheric haze based on these results. Although Smith and Tomasko (1984) found the coloration to reside in both the tropospheric haze and the main ammonia cloud deck, we limited our parameter space here in an effort to find the simplest model that can sufficiently reproduce the data. 


\subsection{Parameter Space Search}

The original $\chi^{2}$ convergence algorithm used by Simon-Miller et al. (2001b) can fit two to four parameters at a time. We found that more than four free variables at once will undermine the fidelity of the minimization. Simon-Miller et al. (2001b) had twelve parameters to fit (four per model layer), and they performed their parameter space search manually. The user selected a small number of free parameters and ran the $\chi^{2}$ convergence algorithm. The user then iterated this process, choosing a new set of free parameters each time, until the user judged that the model provided a satisfactory fit to the data. The parameter space in this work also included twelve free parameters, however the number of data cuts to be modeled was large enough to warrant using an automated search algorithm in conjunction with the $\chi^{2}$ convergence algorithm. This operated in three phases. Phase A determined the appropriate range of values for each parameter. This defined the working search space. Phase $\mathrm{B}$ conducted a grid search of this space, and Phase $\mathrm{C}$ converged on a final solution.

For Phase A, we assumed an initial vertical structure model (see §3.2) and fit two free parameters at a time by an iterative process:

(1) Estimate the increment in the model parameters that would minimize $\chi^{2}$ (Eq. 6). We used a singular value decomposition on a design matrix consisting of the difference between the observations and the current model predictions along with the partial derivatives of the observables with respect to each model parameter (Press, 2002).

(2) Change the model parameters by $90 \%$ of the increments estimated in 
step 1. (Moving $100 \%$ of the distance may overshoot the actual minimum, but choosing to move too slowly would be computationally expensive.)

(3) Run the radiative transfer code on the new model parameters and evaluate $\chi^{2}$.

(4) Repeat steps 1 and 2 until convergence is reached, and save the final parameter values.

(5) Reset the model to the initial values, and proceed to the next parameter pair.

Each parameter was paired more than once, and the value to which a parameter converged depended upon which other parameter was free to vary. For example, $\varpi_{0}(673 \mathrm{~nm})$ in the tropospheric haze may converge to one value when varying simultaneously with the optical depth of the stratospheric haze and a different value when varying with the optical depth of the tropospheric haze. The maximum and minimum values to which each parameter converged in Phase A defined the parameter space for the grid search in Phase B. We explored every free parameter with at least two pairings, but used just $20 \%$ of all possible pairings. Using them all would have greatly increased the required computing time. Run times for each phase on a Pentium 4 processor @ $3.0 \mathrm{GHz}$ ranged from a few minutes to a few hours, depending on the number of samples in the data cut.

In Phase B, we generated model results for a parameter grid that covered the Phase $A$ range. If the range of a given parameter was $<1 \%$, we then used the average value only. The maximum number of grid points, $n_{g}$, was set to $n_{g, \max }=999$ in order to prevent grids from becoming unmanageably large. If Phase A returned $n_{g}>n_{g . \max }$, then the grid resolution for one parameter was reduced. This process was iterated until $n_{g} \leq n_{g, \max }$. The $\chi^{2}$ was evaluated 
for all models in the resulting grid. If the best-fit model did not meet the $\chi^{2}$ cutoff (empirically set to 2.0), we then set this model as the initial conditions for another iteration of Phases A and B.

If the minimum $\chi^{2}$ of the grid was $<2.0$, we then proceeded to Phase $\mathrm{C}$. In this last phase, we ran the parameter-pairing method described in Phase A but without resetting the parameters to the initial values between each iteration. This resulted in a final model in which the parameter values were not quantized, unlike the parameter values in the grid from Phase B.

\subsection{Tests of Methodology and Parameter Assumptions}

An example of the best-fit model to a data cut from the EZ using our methodology (Fig. 8) shows that we find appropriate fits to $I / F$ considering both geometry and wavelength. The parameter space explored by the $\chi^{2}$-minimization algorithm in Phases A and B and the best-fit model are shown in Figure 9. It can be seen that the values checked for each parameter extend above and below the best-fit value.

We wanted to know whether or not our parameter search finds an appropriate $\chi^{2}$ minimum using the initial parameters that we selected. We therefore created a set of synthetic data cuts using parameters that span most of the parameter space of the retrievals from the real data presented in Section 4 . We used one data cut per epoch from each of the six zonal regions (EZ, NEB, NTrZ, NTBs, NTBn, NTZ), which is a total of 24 data cuts. We took the best-fit model result (i.e. synthetic data generated by the radiative transfer code) from each of the three models $\left(P_{3}=[0.4,0.7,1.0]\right.$ bar $)$ and added Gaus- 
sian noise $(\sigma=0.005[I / F])$ to visually match the amount of scatter present in the real data. We retrieved parameters for these synthetic data cuts with the model with the corresponding value of $P_{3}$. For example, our modeling of the real data cut from the EZ in Cube A resulted in three best-fit synthetic data cuts, one for each tropospheric cloud pressure, $P_{3}$. We then added noise to each of these results. We fit a model with $P_{3}=0.4$ bar for the synthetic result with $P_{3}=0.4$ bar, a model with $P_{3}=0.7$ bar on the synthetic result for $P_{3}=0.7$ bar, and a model with $P_{3}=1.0$ bar on the synthetic result for $P_{3}=1.0$ bar. The retrievals (Fig. 10) demonstrate the ability of the $\chi^{2}-$ minimization algorithm to find the correct local minimum (though it may not be a truly global minimum) in the parameter phase space when starting with the initial parameters used for the data retrievals.

One of our simplifying assumptions was to leave the pressure of the stratospheric/tropospheric haze interface $\left(P_{1}\right)$ fixed at 0.1 bar. In reality, this interface undoubtedly varies in height across the planet. Banfield et al. (1998b), Simon-Miller et al. (2001b), and Pérez-Hoyos et al. (2009) report retrievals in the approximate range of $0.1-0.3$ bar. To estimate the effect on $\varpi_{0}(\lambda)$ retrievals due to fixing this parameter value, we generated synthetic data with $P_{1}=0.2$ bar and performed retrievals under our assumption of $P_{1}=0.1$ bar. We created this synthetic data differently from that used in the previous test. We selected the data cut with the most samples from each weather region, changed the best-fit model with $P_{3}=0.7$ bar so that $P_{1}=0.2$ bar, and ran the radiative code on this altered model to generate the synthetic data. In this test, we used all three models to fit the data generated with $P_{3}=0.7$ bar.

Figure 11 shows the retrieved parameters versus the true parameters, and Figure 12 shows the true $\varpi_{0}(\lambda)$ and retrieved $\varpi_{0}(\lambda)$ for the tropospheric haze 
particles from all three models by weather region. From Figure 11 we can see that the retrieved $\varpi_{0}(\lambda)$ values differ at most by $\sim 10 \%$ at 375 and 390 $\mathrm{nm}$ but the differences quickly decrease with longer wavelengths. The largest $\varpi_{0}(\lambda)$ differences are seen in the synthetic data with the darkest particles. The optical depths retrieved differ by as much as about a factor of five for $\tau_{1}$ and about a factor of two for $\tau_{2}$ and $\tau_{3}$. Also note in Figure 12 that in general the three retrieved models (gray lines) have significantly less variance between themselves than the difference between the true $\varpi_{0}(\lambda)$ (black line) and the retrieved ones. This demonstrates that the dependence of the $\varpi_{0}(\lambda)$ retrievals on $P_{3}$ is negligible compared to $P_{1}$ in all regions except the EZ.

Another set of simplifying assumptions was to fix the typical particle radii in each of the three aerosol layers. The particle radii in our models determine the scattering efficiency of aerosols, which is normalized by the scattering efficiency at $502 \mathrm{~nm}$. The optical depths that we report are at this wavelength. If the actual particle radii differ from our assumed distributions, then our assumed $\tau(\lambda)$ relationship will be incorrect. One can compensate for the overor underestimation of the model reflectivity due to an incorrect $\tau(\lambda)$ curve by adjusting $\varpi_{0}(\lambda)$ and still produce satisfactory fits to the data. This is a significant case of degeneracy in radiative transfer models.

To explore this degeneracy, we created synthetic data tests in the same manner as the $P_{1}$ test above. We conducted three separate tests to separate the effects of changing the particle distribution in each aerosol layer. We used our initial models $\left(r_{1}=0.03, r_{2}=0.9, r_{3}=2.0\right)$ to fit data generated with $r_{1}=0.1 \mu \mathrm{m}$ (Figs. 13 and 14), $r_{2}=2.0 \mu \mathrm{m}$ (Figs. 15 and 16), and $r_{3}=4.0 \mu \mathrm{m}$ (Figs. 17 and 18). Fitting with an incorrect particle size distribution had a similar effect for all three aerosol layers. The optical depth results for all three suffered a 
similar level of inaccuracy as the $P_{1}$ test. Also, as with fitting with an incorrect $P_{1}$, the largest $\varpi_{0}(\lambda)$ differences are seen in the synthetic data with the darkest particles and the blue end is worse than the red. The $r_{1}=0.1 \mu \mathrm{m}$ test yielded the most disparate results, with the retrieved $\varpi_{0}(375)$ up to $\sim 50 \%$ too low. However, the retrievals for $\varpi_{0}(410)$ were generally accurate to $\lesssim 6 \%$ and $\varpi_{0}(437-673)$ to $\lesssim 3 \%$.

\section{Results}

We derived best-fit models of $\tau$ and $\varpi_{0}(\lambda)$ for all 1345 data cuts. The data were well fit; the models typically had (reduced) $\chi^{2}$ values of $\sim 1$. Note that degeneracy exists in all radiative transfer solutions to tropospheric structure (West et al., 2004). Therefore, these results are indicative of the true structuresseen through the constraints of our simplified model - and are not to be taken as precise representations.

The results we report for each data cut are the average of all forward models

computed during the $\chi^{2}$-minimization routine that have $\chi^{2}<2 \chi_{\min }^{2}$, where $\chi_{\min }^{2}$ is the value for the best fit. Most data cuts were well fit by the parameter space search using all three initial models, which varied only in the pressure level of the interface between the tropospheric haze and cloud. Therefore, the results presented typically contain contributions from at least one model from each vertical structure. 


\subsection{Aerosol Optical Depths}

The particle color retrievals are dependent upon the optical depth retrievals. Much data exist concerning Jupiter's vertical aerosol structure, and little data exist for the particle color-none of which describes the global distribution. Therefore, we first considered whether or not the vertical aerosol structure retrievals provided a realistic physical context for the particle color retrievals.

The optical depths retrieved for each aerosol layer are presented in Table 6 and Fig. 19-21 for each weather region. The number of data cuts per weather region is noted in each panel. The stratospheric haze (Fig. 19) has $\tau_{1}<0.35$ in all weather regions and a typical value of $\tau_{1}=\sim 0.15$. This is consistent with the Banfield et al. (1998b) SSI retrievals of $\tau_{1}=\sim 0.1$ and the value of $\tau_{1}(660)=0.2$ found by Sromovsky and Fry (2002) using fits to center-to-limb brightness curves from WFPC2 observations. All zones have greater $\tau_{1}$ than the belts and the vortices, with the exception of the red annulus of Oval BA, which was comparable to the zones. The three lowest $\tau_{1}$ values were found in the NEB, NEB1, and GRS center.

The optical depths of the tropospheric haze (Fig. 20 and Table 6) only deviate slightly from the initial value of $\tau_{2}=4$, except for the NEB and NEB1, where optical depths increase to 15. Except for these high values in the NEB and NEB1, $\tau_{2}$ fell within the range of $2 \gtrsim \tau_{2} \gtrsim 8$ reported by West et al. (2004). Locations with redder observed colors (e.g. belts, GRS center, Oval 2) had slightly higher $\tau_{2}$ overall, while locations with the whitest observed colors (e.g. EZ, NTrZ, A5) had slightly lower $\tau_{2}$. This is consistent with Irwin and Dyudina (2002), whose radiative transfer models indicated "that the haze near 
the tropopause $(0.26$ bars) is denser above the belt than above the zone."

The horizontal variations in our $\tau_{1}$ and $\tau_{2}$ retrievals are fairly small. When considered together, they suggest that Jupiter would be nearly uniformly bright in $\mathrm{CH}_{4}$ absorption bands. Observations in these bands, however, show that zones and the GRS appear brighter than their surroundings (e.g. Karkoschka, 1998). This discrepancy between our model retrievals and observations in $\mathrm{CH}_{4}$ absorption bands is not surprising, as our data did not include $\mathrm{CH}_{4}$ filters.

The optical depths of the tropospheric cloud (Fig. 21 and Table 6) exhibit small differences between belts and zones. The NTrZ and NTZ both have a greater average $\tau_{3}$ than the belts, but the EZ does not. These results do not follow the general consensus that belts contain significantly less opacity in the tropospheric cloud deck than zones do. Irwin et al. (2001) suggest, however, "that the main source of the near-IR reflectivity variability, observed to be anticorrelated with 5 - $\mu \mathrm{m}$ brightness, is due to opacity changes of cloud layers lying at pressures between 1 and 2 bar, and not to variability of the higher altitude ammonia cloud." Thus, our near-UV and visible data may not be sensitive to the altitudes at which the belt/zone opacity differences are greatest. Retrieved values for individual locations differ by $\sim 15$ within each weather region, with most regions in the range of $18 \gtrsim \tau_{3} \gtrsim 50$. However, $\sim 50 \%$ of the locations in both the NEB and NEB1 have significantly lower optical depths $\left(\tau_{3} \sim 10\right)$ than all other weather regions.

An interesting result in the optical depth retrievals is that the tropospheric haze is highly positively correlated with the optical depth of the cloud in many of the weather regions (Fig. 22 and Table 6), with an overall correlation coefficient of 0.147 . This correlation is strong in small weather regions (e.g. 
0.995 for the NEB1 and 0.789 for the GRS center) whose the data cuts have inherently limited longitudinal coverage, but it is also equally present in some zonal regions as well (e.g. 0.974 for the NEB and 0.818 for the NTBn). Our interpretation is that the correlations are indicative of a degeneracy in the model, which is easily accounted for by the poor vertical sensitivity of the models due to the lack of data at $\mathrm{CH}_{4}$ absorption bands. Instead of physically interpreting the optical depths of the two layers independently, it may be necessary to consider differences in their ratios when looking for real horizontal variations in the model results.

\subsection{Single Scattering Albedos of Tropospheric Haze Aerosols}

We retrieved the tropospheric haze particle color from a globally representative sample of jovian locations (Table 7 ). Figure 23 shows the mean $\varpi_{0}(\lambda)$ from $375-673 \mathrm{~nm}$ for all data cuts, with the initial $\varpi_{0}(\lambda)$ for comparison. Figure 24 presents the results by weather region. Again, this is the first global analysis with multiwavelength retrievals, so we compare our results with the global analysis of a single wavelength by Simon-Miller et al. (2001b) and the multiwavelength analysis of Oval BA and its immediate surroundings by PérezHoyos et al. (2009).

Our values for $\varpi_{0}(410 \mathrm{~nm})$ are in good agreement with the range found by Simon-Miller et al. (2001b) of 0.922-1.0 in their analysis of Galileo SSI data. Our results are also consistent with $\varpi_{0}$ values for filters F330W, F435W, and F658N reported by Pérez-Hoyos et al. (2009) in their study of oval BA (see their Fig. 9 and 11). Their $\varpi_{0}(\lambda)$ results were slightly higher; however, they assumed a darker stratospheric haze $\left(\varpi_{0}=0.95\right)$. 
We subtracted the mean $\varpi_{0}(\lambda)$ (Fig. 23, solid line) and ran a PCA in order to explore the spectral shapes that contribute to the variance about the mean $\varpi_{0}(\lambda)$. We pause to note that all PCA results reported in this work (the $\varpi_{0}(\lambda)$ PCA here and the $I / F(\lambda)$ PCA in $\left.\S 5.3\right)$ hold true for two different PCA implementations. In the first case, all 1345 data cuts are included in the PCA. In the second case, the data cuts in each weather region are averaged and the PCA is run on those 14 averages. This demonstrates that our disparate number of data cuts between weather regions does not bias the PCA results. We present only the results for PCAs of all 1345 data cuts.

The $\varpi_{0}(\lambda)$ PCA yielded two significant components, PC1 and PC2, which account for $>99 \%$ of the total variance (Fig. 25, top panel). The higherorder PCs (Fig. 25, bottom panel) describe an amount of spectral variance much smaller than our confidence in the retrievals due to the degeneracy as described in Section 3.4. We also view with suspicion the possibility of PC2 describing a true physical difference between chromophore populations. PC2 is primarily an anti-correlation between $\varpi_{0}(375)$ and $\varpi_{0}(410-502)$, and the value of $\varpi_{0}(375)$ was the most susceptible to inaccurate retrievals of the $\varpi_{0}$ parameters $(\S 3.4)$.

The $\varpi_{0}(\lambda)$ for a location is the sum of the mean $\varpi_{0}(\lambda)$ and each PC multiplied by its amplitude. Figure 26 shows the PC1 and PC2 amplitudes by weather region. As the amplitude of $\mathrm{PC} 1$ decreases (i.e. moves in the direction of negative amplitudes), it describes increasingly redder particle colors that are also darker at all wavelengths. Note that locations with the highest positive values of PC1 still have a $\varpi_{0}(\lambda)$ that is red. No location has a PC1 value high enough to have a blue $\varpi_{0}(\lambda)$ once the mean $\varpi_{0}(\lambda)$ is added back. Positive values of PC2 describe particles with a decreased ratio of $\varpi_{0}(375)$ to $\varpi_{0}(410-$ 
502) than the mean and vice-versa. The data cuts with the strongest PC2 contribution to their $\varpi_{0}(\lambda)$ belong to the NEB and NEB1, which is another reason to doubt the existence of a physical cause behind $\mathrm{PC} 2$. The retrievals of $\varpi_{0}(375)$ for these two regions were affected by degeneracy with $P_{1}$ and $r_{1-3}$ more than most other regions (Figs. 12, 14, 16, and 18).

\section{Discussion}

\subsection{Chromophore Particle Colors}

The spectra of the chromophores were quite similar for different areas that spanned a large range of observed coloration. All locations had $\varpi_{0}(\lambda)$ with a positive slope, and the slope decreases with increasing wavelength. Our results are therefore consistent with the presence of an ubiquitous blue absorber in Jupiter's upper troposphere, which was suggested by Simon-Miller et al. (2001a). None of the data cuts had $\varpi_{0}(\lambda)$ with a dramatically different shape than the mean. We empirically found a functional form that accurately describes the $\varpi_{0}(\lambda)$ from all retrievals:

$$
\varpi_{0}(\lambda)=1-A \exp (-B \lambda)
$$

The best-fit parameters for the mean $\varpi_{0}(\lambda)$ are $A=25.4$ and $B=0.0149$ for $\lambda$ in units of nm (Fig. 23, dashed line), with an RMS error of 0.00174 . Not only does this functional form fit the mean $\varpi_{0}(\lambda)$, but it also fits each individual $\varpi_{0}(\lambda)$ retrieval (fitting parameters $A$ and $B$ separately for each of the 1345 spectra) with an average and standard deviation of RMS error of $0.00195 \pm 0.00062$. Table 8 contains the average and standard deviation of the 
parameter fits for all individual $\varpi_{0}(\lambda)$ retrievals by weather region.

\subsection{Chromophore Populations}

For both the $\varpi_{0}(\lambda)$ and $I / F_{0}(\lambda)$ PCAs, one chromophore alone can explain both the mean spectral shape and PC1 by horizontal variations in the chromophore's concentration. If there is an underlying physical cause for $\varpi_{0}(\lambda)$ PC2, then it may be caused by one or more of three possibilities: (1) spatial variations in the vertical aerosol structure that affect $I / F_{0}(\lambda)$, which are not explored in the simplified model used here to obtain $\varpi_{0}(\lambda) ;(2)$ spatial variations in the physical properties of the chromophore particles, such as radius, shape, temperature, pressure, and UV exposure; or (3) spatial variations in the concentration of a second chromophore.

There are two clues to the interpretation of PC1. The first is the fact that locations that appear more red than others in observed color (i.e. more negative $\frac{d^{2} I / F_{0}(\lambda)}{d \lambda^{2}}$ ) also have $\varpi_{0}(\lambda)$ that are more red (i.e. more negative $\frac{d^{2} \varpi_{0}(\lambda)}{d \lambda^{2}}$ ) and darker at all wavelengths. Although we intuitively expected redder particles producing redder observed colors, it was not absolutely necessary. The second clue is that the optical depth of the tropospheric cloud is positively correlated with the amplitude of $\mathrm{PC} 1$ with an overall correlation coefficient of 0.780 (Fig. 27). This indicates a degeneracy between $\tau_{3}$ and brightness of chromophore color and/or a real relationship between the two. If this is merely a degeneracy, then why did locations with redder observed color all have redder $\varpi_{0}(\lambda)$ retrievals? On the other hand, if it is a real relationship, why is the correlation so strong across all retrievals? 
There is a physically realistic scenario that could produce a strong correlation. It is probable that locations with higher $\tau_{3}$ would also have increased upwelling of $\mathrm{NH}_{3}$ gas. This might cause an increased mixing ratio of $\mathrm{NH}_{3}$ ice to chromophore particles in the tropospheric haze and perhaps increased coating of chromophores with ice in the vicinity of the ammonia cloud, both of which would brighten our retrieved $\varpi_{0}(\lambda)$. These possibilities-a degeneracy, an increased mixing ratio of $\mathrm{NH}_{3}$ ice to chromophores, and an increased ice coating of chromophores - all lead us to conclude that our $\varpi_{0}(\lambda)$ results are consistent with only one chromophore population. We are unable to distinguish between chromophore populations, because that requires a high level of certainty concerning cloud heights, optical depths, and particle radii that are too poorly constrained in this current study.

\subsection{Comparison between Statistical Analyses of $I / F_{0}(\lambda)$ and $\varpi_{0}(\lambda)$}

We examined how this $\varpi_{0}(\lambda)$ PCA compares to an analysis of $I / F_{0}(\lambda)$ in order to determine the extent to which $I / F_{0}(\lambda)$ variations can be used as a proxy for $\varpi_{0}(\lambda)$ variations. PCA of the mean-subtracted $I / F_{0}(375-673)$ also yielded two significant components with similar amounts of variance to the $\varpi_{0}(\lambda)$ PCA (Fig. 28, top panel) and higher-order PCs with variances $<1 \%$ (Fig. 28, bottom panel). Locations with negative values of PC1 have darker and redder observed colors than the mean $I / F_{0}(\lambda)$ (Fig. 4, solid line), and those with negative values of $\mathrm{PC} 2$ have particles with an increased ratio of 375-410-nm absorption to 469-673-nm absorption than the mean.

Are the largest variations in $I / F_{0}(\lambda)$ correlated with those of $\varpi_{0}(\lambda)$ ? Figure 29 compares the amplitudes of the $I / F_{0}(\lambda)$ PCs and $\varpi_{0}(\lambda)$ PCs for each jovian 
location. The strong correlation between the PC amplitudes demonstrates the potential usefulness of reflectance spectra for the purpose of determining the number of statistically independent chromophores. However, these components may not correspond to identical underlying phenomena, as evidenced by the fact that the relationship between the PCs is not one-to-one but contains obvious structure. Additionally, the $I / F_{0}(\lambda)$ PCs have significantly different spectral shapes from the $\varpi_{0}(\lambda)$ PCs. Thus, although the $I / F_{0}(\lambda)$ PCs do reveal spectral characteristics of a chromophore's observed color, they do not reveal the spectral characteristics of a chromophore's particle color.

\section{Conclusions}

In this study we derived and statistically analyzed horizontal variations of $\varpi_{0}(\lambda)$ in Jupiter's troposphere using multispectral HST WFPC2 images. The locations in our sample were representative of the typical range of the observed

jovian color. Our radiative transfer models of $I / F$ yielded the first global results for the spectra of chromophore particle colors at multiple near-UV and visible wavelengths. An empirical functional form (Eq. 7) fits the $\varpi_{0}(\lambda)$ retrievals very well.

Although derived values for $\varpi_{0}(\lambda)$ from models are dependent upon the assumed vertical aerosol structure, we do not solve for all parameters of that structure. We assumed the pressure levels of the aerosol layers due to the limited vertical sensitivity inherent in the data. We derived optical depths and chromophore spectral shapes in the near-UV and visible wavelength regime for 1345 individual planet locations, assuming the chromophores affecting these wavelengths were located solely in the tropospheric haze. 
We characterized the spatial variations in particle color via $\varpi_{0}(\lambda)$ PCA and found that two PCs account for $>99 \%$ of the total variance in the spectral shapes of chromophores. Our results are consistent with the conclusion of Simon-Miller et al. (2001a) that one or two chromophores are necessary to explain color variations in the jovian atmosphere. The $I / F_{0}(\lambda)$ PCA in this work makes a stronger case than Simon-Miller et al. (2001a) for the upper limit to the number of chromophores because of our greater spectral coverage. However, PC2 in the $\varpi_{0}(\lambda)$ PCA was likely describing spectral shapes that arose due to inaccurate retrievals from degeneracy in the radiative transfer model parameters $-\varpi_{0}(\lambda)$ was particularly affected by the choices of the pressures and particle radii associated with each aerosol layer. We also find that modeling the physical properties that contribute to the observed $I / F(\lambda)$ provides a distinctly different context for characterizing chromophore spectra than PCA of $I / F_{0}(\lambda)$. We recognize the need for additional multispectral studies that sample the continuum below $\sim 600 \mathrm{~nm}$ as well as obtain observations in $\mathrm{CH}_{4}$ absorption bands of various strengths, which will further constrain the radiative transfer retrievals while making the context more physically appropriate.

The particle spectra presented in this work represent a large portion of the global distribution of chromophore(s) on Jupiter. Yet, we cannot chemically identify the chromophore(s) using our derived particle spectra as they contain no discrete absorption features - only broad changes across the entire nearUV and visible regime. It is possible that an absorption feature may exist in the gap in our coverage between $502 \mathrm{~nm}$ and $673 \mathrm{~nm}$, which is precisely the spectral region identified by Lebofsky and Fegley (1976) where both $\mathrm{H}_{2} \mathrm{~S}$ and $\mathrm{NH}_{4} \mathrm{SH}$ ices develop an absorption feature $\sim 100-\mathrm{nm}$ wide centered at $\sim 600 \mathrm{~nm}$ when irradiated with UV light (see also Sill, 1973). Moreover, recent work by 
Sugiyama et al. (2009), Sromovsky and Fry (2010a), and Sromovsky and Fry (2010b) indicates $\mathrm{NH}_{4} \mathrm{SH}$ particles may be widespread in Jupiter's upper troposphere. Future chromophore studies should include spectral coverage from $500-700 \mathrm{~nm}$ to search for these ice features. We also encourage the continued study of chromophore candidates (see West et al., 1986) in the laboratory to provide a database of their indices of refraction - as a function of wavelength, temperature, pressure, irradiation, etc.-to achieve the ultimate goal of identifying the chemical species and the local environmental conditions necessary to produce the observed jovian colors that so deeply intrigue us.

\section{Acknowledgements}

The authors would like to thank two anonymous reviewers for constructive feedback, which greatly enhanced this work. We also wish to acknowledge Michael Roman for contributions to the radiative transfer code. This work was supported by NASA's Planetary Atmospheres Program through grant number NNX08AF53A. This work is based on observations made with the NASA/ESA Hubble Space Telescope, obtained at the Space Telescope Science Institute, which is operated by the Association of Universities for Research in Astronomy, Inc, under NASA contract NAS 5-26555. These observations are associated with program \#GO/DD11498.

\section{References}

Banfield, D., Conrath, B. J., Gierasch, P. J., Nicholson, P. D., Matthews, K.,

Jul. 1998a. Near-IR Spectrophotometry of Jovian Aerosols-Meridional and 
Vertical Distributions. Icarus 134, 11-23.

Banfield, D., Gierasch, P. J., Bell, M., Ustinov, E., Ingersoll, A. P., Vasavada, A. R., West, R. A., Belton, M. J. S., Sep. 1998b. Jupiter's Cloud Structure from Galileo Imaging Data. Icarus 135, 230-250.

Beebe, R. F., Hockey, T. A., Jul. 1986. A comparison of red spots in the atmosphere of Jupiter. Icarus 67, 96-105.

de Pater, I., Fletcher, L. N., Pérez-Hoyos, S., Hammel, H. B., Orton, G. S., Wong, M. H., Luszcz-Cook, S., Sánchez-Lavega, A., Boslough, M., Dec. 2010a. A multi-wavelength study of the 2009 impact on Jupiter: Comparison of high resolution images from Gemini, Keck and HST. Icarus 210, 722-741. de Pater, I., Wong, M. H., Marcus, P., Luszcz-Cook, S., Ádámkovics, M., Conrad, A., Asay-Davis, X., Go, C., Dec. 2010b. Persistent rings in and around Jupiter's anticyclones - Observations and theory. Icarus 210, 742762.

Dyudina, U. A., Ingersoll, A. P., Danielson, G. E., Baines, K. H., Carlson, R. W., The Galileo Nims, SSI Teams, Apr. 2001. Interpretation of NIMS and SSI Images on the Jovian Cloud Structure. Icarus 150, 219-233.

Gonzaga, S., Biretta, J., Apr. 2009. WFPC2 F343N Filter Throughput Decline. Tech. rep.

Gonzaga, S., Brammer, G., Heyer, I., Lubin, L., Baggett, S., Ritchie, C., Casertano, S., Whitmore, B., Mutchler, M., Koekemoer, A., Oct. 2006. WFPC2 Standard Star Monitoring memo. Tech. rep.

Hansen, J. E., Travis, L. D., Oct. 1974. Light scattering in planetary atmospheres. Space Science Reviews 16, 527-610.

Irwin, P. G. J., Dyudina, U., Mar. 2002. The Retrieval of Cloud Structure Maps in the Equatorial Region of Jupiter Using a Principal Component Analysis of Galileo/NIMS Data. Icarus 156, 5263 . 
Irwin, P. G. J., Weir, A. L., Taylor, F. W., Calcutt, S. B., Carlson, R. W., Feb. 2001. The Origin of Belt/Zone Contrasts in the Atmosphere of Jupiter and Their Correlation with 5- $\mu \mathrm{m}$ Opacity. Icarus 149, 397-415.

Karkoschka, E., May 1998. Methane, Ammonia, and Temperature Measurements of the Jovian Planets and Titan from CCD-Spectrophotometry. Icarus $133,134-146$.

Karkoschka, E., Tomasko, M. G., Feb. 2010. Methane absorption coefficients for the jovian planets from laboratory, Huygens, and HST data. Icarus 205, $674-694$.

Lebofsky, L. A., Fegley, Jr., M. B., Jul. 1976. Laboratory reflection spectra for the determination of chemical composition of ice bodies. Icarus 28, 379-387. Martonchik, J. V., Orton, G. S., Appleby, J. F., Feb. 1984. Optical properties of NH3 ice from the far infrared to the near ultraviolet. Applied Optics 23, $541-547$.

Mishchenko, M. I., Dlugach, Z. M., Yanovitskij, E. G., Zakharova, N. T., Dec. 1999. Bidirectional reflectance of flat, optically thick particulate layers: an efficient radiative transfer solution and applications to snow and soil surfaces. J. Quant. Spectrosc. Radiat. Transfer 63, 409-432.

Murtagh, F., Heck, A., 1987. Multivariate Data Analysis. Reidel, Dordrecht. Owen, T., Terrile, R. J., Sep. 1981. Colors on Jupiter. J. Geophys. Res. 86, $8797-8814$

Pérez-Hoyos, S., Sánchez-Lavega, A., Hueso, R., García-Melendo, E., Legarreta, J., Oct. 2009. The jovian anticyclone BA. III. Aerosol properties and color change. Icarus 203, 516-530.

Preisendorfer, R. W., Mobley, C. D., 1988. Principal Component Analysis in Meteorology and Oceanography. Elsevier, Amsterdam.

Press, W. H., 2002. Numerical recipes in $\mathrm{C}++$ : the art of scientific computing. 
Sill, G. T., 1973. Reflection spectra of solids of planetary interest. Communications of the Lunar and Planetary Laboratory 10, 1-7.

Simon-Miller, A. A., Banfield, D., Gierasch, P. J., Jan. 2001a. An HST Study of Jovian Chromophores. Icarus 149, 94-106.

Simon-Miller, A. A., Banfield, D., Gierasch, P. J., Dec. 2001b. Color and the Vertical Structure in Jupiter's Belts, Zones, and Weather Systems. Icarus $154,459-474$.

Simon-Miller, A. A., Chanover, N. J., Orton, G. S., Sussman, M., Tsavaris, I. G., Karkoschka, E., Dec. 2006. Jupiter's White Oval turns red. Icarus $185,558-562$.

Simon-Miller, A. A., Gierasch, P. J., Nov. 2010. On the long-term variability of Jupiter's winds and brightness as observed from Hubble. Icarus 210, 258 269.

Smith, P. H., Tomasko, M. G., Apr. 1984. Photometry and polarimetry of Jupiter at large phase angles. II - Polarimetry of the south tropical zone, south equatorial belt, and the polar regions from the Pioneer 10 and 11 missions. Icarus 58, 35-73.

Sromovsky, L. A., Fry, P. M., Jun. 2002. Jupiter's Cloud Structure as Constrained by Galileo Probe and HST Observations. Icarus 157, 373-400.

Sromovsky, L. A., Fry, P. M., Nov. 2010a. The source of 3- $\mu \mathrm{m}$ absorption in Jupiter's clouds: Reanalysis of ISO observations using new $\mathrm{NH}_{3}$ absorption models. Icarus 210, 211-229.

Sromovsky, L. A., Fry, P. M., Nov. 2010b. The source of widespread 3- $\mu \mathrm{m}$ absorption in Jupiter's clouds: Constraints from 2000 Cassini VIMS observations. Icarus 210, 230-257.

Sugiyama, K., Odaka, M., Nakajima, K., Hayashi, Y., 2009. Development of a Numerical Model to investigate the Moist Convection in Jupiter's Atmo- 
sphere. Nagare Multimedia.

Thompson, W. R., 1990. Global four-band spectral classification of Jupiter's clouds - Color/albedo units and trends. International Journal of Supercomputer Applications 4, 48-65.

von Zahn, U., Hunten, D. M., Lehmacher, G., Sep. 1998. Helium in Jupiter's atmosphere: Results from the Galileo probe helium interferometer experiment. J. Geophys. Res. 103, 22815-22830.

West, R. A., Baines, K. H., Friedson, A. J., Banfield, D., Ragent, B., Taylor, F. W., 2004. Jovian clouds and haze. In: Bagenal, F., Dowling, T. E., \& McKinnon, W. B. (Ed.), Jupiter. The Planet, Satellites and Magnetosphere. pp. $79-104$.

West, R. A., Strobel, D. F., Tomasko, M. G., Mar. 1986. Clouds, aerosols, and photochemistry in the Jovian atmosphere. Icarus 65, 161-217.

Wong, M. H., Mahaffy, P. R., Atreya, S. K., Niemann, H. B., Owen, T. C., Sep. 2004. Updated Galileo probe mass spectrometer measurements of carbon, oxygen, nitrogen, and sulfur on Jupiter. Icarus 171, 153-170.

* Partly based on observations obtained from the data archive at the Space Telescope Science Institute. STScI is operated by the Association of Universities for Research in Astronomy, Inc., under NASA contract NAS 5-26555. 
Table 1. Summary of HST Observations

\begin{tabular}{|c|c|c|c|}
\hline $\begin{array}{c}\text { UT Date } \\
\text { (YYYY-MM-DD) }\end{array}$ & $\begin{array}{l}\text { Spectral } \\
\text { Cube ID }\end{array}$ & $\begin{array}{l}\text { WFPC2 } \\
\text { Filter }\end{array}$ & Image \# \\
\hline \multirow[t]{22}{*}{$2008-05-15$} & A & F255W & ub060105 \\
\hline & $\mathrm{A}$ & $\mathrm{F} 343 \mathrm{~N}$ & ub060106 \\
\hline & A & F $375 \mathrm{~N}$ & ub060107 \\
\hline & $\mathrm{A}$ & F390N & ub060108 \\
\hline & $A$ & F410M & ub060102 \\
\hline & $\mathrm{A}$ & F410M & ub06010d \\
\hline & A & $\mathrm{F} 437 \mathrm{~N}$ & ub060109 \\
\hline & A & $\mathrm{F} 469 \mathrm{~N}$ & ub06010a \\
\hline & $\mathrm{A}$ & F502N & ub06010b \\
\hline & A & F673N & ub060101 \\
\hline & A & F673N & ub06010c \\
\hline & $B$ & $\mathrm{~F} 255 \mathrm{~W}$ & ub060205 \\
\hline & $B$ & F343N & ub060206 \\
\hline & B & F375N & ub060207 \\
\hline & $\mathrm{B}$ & F390N & ub060208 \\
\hline & B & F410M & ub060202 \\
\hline & B & F410M & ub06020d \\
\hline & $B$ & $\mathrm{~F} 437 \mathrm{~N}$ & ub060209 \\
\hline & B & $\mathrm{F} 469 \mathrm{~N}$ & ub06020a \\
\hline & $\mathrm{B}$ & $\mathrm{F} 502 \mathrm{~N}$ & ub06020b \\
\hline & $B$ & F673N & ub060201 \\
\hline & $\mathrm{B}$ & F673N & ub06020c \\
\hline \multirow[t]{10}{*}{$2008-06-28$} & $\mathrm{C}$ & F255W & ub060403 \\
\hline & $\mathrm{C}$ & F $343 N$ & ub060404 \\
\hline & $\mathrm{C}$ & $\mathrm{F} 375 \mathrm{~N}$ & ub060405 \\
\hline & $\mathrm{C}$ & F390N & ub060406 \\
\hline & $\mathrm{C}$ & F410M & ub060402 \\
\hline & $\mathrm{C}$ & $\mathrm{F} 437 \mathrm{~N}$ & ub060407 \\
\hline & C & $\mathrm{F} 469 \mathrm{~N}$ & ub060408 \\
\hline & $\mathrm{C}$ & $\mathrm{F} 502 \mathrm{~N}$ & ub060409 \\
\hline & $\mathrm{C}$ & F673N & ub060401 \\
\hline & $\mathrm{C}$ & F673N & $\mathrm{ub} 06040 \mathrm{c}$ \\
\hline \multirow[t]{10}{*}{$2008-07-08$} & $\mathrm{D}$ & F255W & ub060609 \\
\hline & $\mathrm{D}$ & F343N & ub060604 \\
\hline & $\mathrm{D}$ & F375N & ub060605 \\
\hline & $\mathrm{D}$ & F390N & ub060606 \\
\hline & $\mathrm{D}$ & F410M & ub060602 \\
\hline & $\mathrm{D}$ & $\mathrm{F} 437 \mathrm{~N}$ & ub060607 \\
\hline & $\mathrm{D}$ & $\mathrm{F} 469 \mathrm{~N}$ & ub060608 \\
\hline & $\mathrm{D}$ & $\mathrm{F} 502 \mathrm{~N}$ & ub060603 \\
\hline & $\mathrm{D}$ & F673N & ub060601 \\
\hline & $\mathrm{D}$ & F673N & ub06060c \\
\hline
\end{tabular}


Table 2. Weather Regions

\begin{tabular}{|c|c|c|c|c|c|c|c|c|}
\hline Region & $\begin{array}{c}\text { Feature } \\
\text { Type }\end{array}$ & $\begin{array}{c}\text { Planetographic } \\
\text { Latitude }\end{array}$ & $\begin{array}{l}\text { System III } \\
\text { Longitude }\end{array}$ & $\begin{array}{l}\text { Longitude } \\
\text { Range }\end{array}$ & $\begin{array}{c}\mu \\
\text { Range }\end{array}$ & $\begin{array}{c}\mu_{0} \\
\text { Range }\end{array}$ & $\begin{array}{c}\text { Samples per } \\
\text { Data Cut }\end{array}$ & $\begin{array}{l}\text { Data } \\
\text { Cuts }\end{array}$ \\
\hline $\mathrm{EZ}$ & zone & $-0.0 \pm 2.0$ & $122.5 \pm 19.2$ & $44.2 \pm 4.4$ & $0.21 \pm 0.04$ & $0.27 \pm 0.03$ & $414 \pm 68$ & 12 \\
\hline NEB & belt & $11.2 \pm 0.6$ & $122.5 \pm 19.2$ & $32.1 \pm 7.5$ & $0.15 \pm 0.03$ & $0.16 \pm 0.03$ & $301 \pm 64$ & 12 \\
\hline$N \operatorname{TrZ}$ & zone & $21.6 \pm 0.4$ & $122.5 \pm 19.2$ & $46.5 \pm 6.1$ & $0.21 \pm 0.05$ & $0.26 \pm 0.03$ & $467 \pm 69$ & 12 \\
\hline NTBs & belt & $24.5 \pm 0.7$ & $122.5 \pm 19.2$ & $41.4 \pm 7.7$ & $0.18 \pm 0.04$ & $0.22 \pm 0.04$ & $423 \pm 74$ & 12 \\
\hline NTBn & belt & $27.3 \pm 0.8$ & $122.5 \pm 19.2$ & $45.9 \pm 3.2$ & $0.19 \pm 0.03$ & $0.24 \pm 0.03$ & $477 \pm 33$ & 12 \\
\hline NTZ & zone & $33.9 \pm 0.6$ & $122.5 \pm 19.2$ & $44.6 \pm 11.0$ & $0.20 \pm 0.06$ & $0.21 \pm 0.04$ & $392 \pm 78$ & 12 \\
\hline GRS bright annulus & oval & $-22.1 \pm 2.1$ & $111.3 \pm 7.6$ & $7.6 \pm 3.9$ & $0.09 \pm 0.04$ & $0.08 \pm 0.04$ & $56 \pm 26$ & 566 \\
\hline GRS center & oval & $-23.1 \pm 0.8$ & $111.6 \pm 6.4$ & $4.7 \pm 1.1$ & $0.08 \pm 0.03$ & $0.07 \pm 0.04$ & $52 \pm 11$ & 115 \\
\hline GRS red annulus & oval & $-22.2 \pm 3.1$ & $114.4 \pm 7.5$ & $7.7 \pm 5.7$ & $0.11 \pm 0.04$ & $0.11 \pm 0.05$ & $35 \pm 17$ & 180 \\
\hline BA center & oval & $-33.4 \pm 0.9$ & $118.4 \pm 5.0$ & $2.1 \pm 0.7$ & $0.07 \pm 0.02$ & $0.10 \pm 0.04$ & $27 \pm 7$ & 98 \\
\hline BA red annulus & oval & $-33.4 \pm 1.2$ & $117.9 \pm 5.6$ & $6.0 \pm 1.8$ & $0.08 \pm 0.02$ & $0.10 \pm 0.05$ & $32 \pm 9$ & 190 \\
\hline Oval 2 & oval & $-24.4 \pm 0.6$ & $129.6 \pm 3.2$ & $1.9 \pm 0.4$ & $0.03 \pm 0.01$ & $0.07 \pm 0.03$ & $23 \pm 4$ & 52 \\
\hline NEB1 & oval & $15.5 \pm 0.5$ & $89.4 \pm 4.0$ & $1.3 \pm 0.3$ & $0.13 \pm 0.08$ & $0.17 \pm 0.06$ & $21 \pm 2$ & 36 \\
\hline A5 & oval & $-40.7 \pm 0.7$ & $92.5 \pm 0.0$ & $2.2 \pm 0.5$ & $0.03 \pm 0.00$ & $0.05 \pm 0.00$ & $30 \pm 5$ & 36 \\
\hline
\end{tabular}

All values following a " \pm " sign are standard deviations (not uncertainties) for that region's data cuts. 
Table 3. HST WFPC2 Filters and Beer's Law Parameter Fits

\begin{tabular}{cccc} 
Filter & $\begin{array}{c}\text { Weighted Mean } \\
\text { Wavelength (nm) }\end{array}$ & $\begin{array}{c}\text { Absorption } \\
\text { Parameter }^{a}\end{array}$ & $\begin{array}{c}\text { Scattering } \\
\text { Parameter }^{a}\end{array}$ \\
\hline F255W & 260.490 & $2.7 \mathrm{E}-07$ & 1.842 \\
F343N & 343.416 & $9.5 \mathrm{E}-06$ & 0.607 \\
F375N & 373.988 & $1.1 \mathrm{E}-05$ & 0.432 \\
F390N & 389.319 & $1.1 \mathrm{E}-05$ & 0.367 \\
F410M & 408.811 & $1.0 \mathrm{E}-05$ & 0.301 \\
F437N & 436.922 & $9.5 \mathrm{E}-05$ & 0.230 \\
F469N & 469.439 & $2.7 \mathrm{E}-05$ & 0.173 \\
F502N & 501.244 & $1.9 \mathrm{E}-04$ & 0.133 \\
F673N & 673.229 & $4.3 \mathrm{E}-03$ & 0.041
\end{tabular}

a The absorption and scattering parameter values are fits to the calculated filter transmissivities (see Fig. 6) with this functional form of Beer's Law: $T=\exp (-2 A p)$, where $T$ is (1-extinction) at the surface for a beam of light traveling down to the given pressure and back, $p$ is the pressure, and $A$ is the absorption or scattering parameter. 


\section{Table 4. Variability Flags for Model Parameters}

\begin{tabular}{cccc}
\hline & Stratospheric & Tropospheric & Tropospheric \\
Parameter & Haze & Haze & Sheet Cloud \\
\hline$P$ & 0 & 0 & 2 \\
$\tau(502)$ & 1 & 1 & 1 \\
$r_{0}$ & 0 & 0 & 0 \\
$\varpi_{0}(255)$ & 1 & 3 & 0 \\
$\varpi_{0}(343)$ & 1 & 3 & 0 \\
$\varpi_{0}(375)$ & 0 & 1 & 0 \\
$\varpi_{0}(390)$ & 0 & 1 & 0 \\
$\varpi_{0}(410)$ & 0 & 1 & 0 \\
$\varpi_{0}(437)$ & 0 & 1 & 0 \\
$\varpi_{0}(469)$ & 0 & 1 & 0 \\
$\varpi_{0}(502)$ & 0 & 1 & 0 \\
$\varpi_{0}(673)$ & 0 & 1 & 0
\end{tabular}

Flag definitions: 0 denotes fixed parameters, 1 denotes parameters free to vary, 2 denotes a pressure parameter fixed to the layer above (creating an infinitesimally thin layer), and 3 denotes $\varpi_{0}$ parameters fixed to the filter redward in the same layer. 


\section{Table 5. Initial Values for Model Parameters}

\begin{tabular}{cccc} 
Parameter & $\begin{array}{c}\text { Stratospheric } \\
\text { Haze }\end{array}$ & $\begin{array}{c}\text { Tropospheric } \\
\text { Haze }\end{array}$ & $\begin{array}{c}\text { Tropospheric } \\
\text { Sheet Cloud }\end{array}$ \\
\hline$P[\mathrm{bar}]$ & 0.100 & $0.4,0.7,1.0$ & $0.4,0.7,1.0$ \\
$\tau(502)$ & 0.200 & 4.000 & 20.00 \\
$r_{0}[\mu \mathrm{m}]$ & 0.030 & 0.900 & 2.000 \\
$\varpi_{0}(255)$ & 0.900 & 0.920 & 1.000 \\
$\varpi_{0}(343)$ & 0.900 & 0.920 & 1.000 \\
$\varpi_{0}(375)$ & 1.000 & 0.920 & 1.000 \\
$\varpi_{0}(390)$ & 1.000 & 0.940 & 1.000 \\
$\varpi_{0}(410)$ & 1.000 & 0.950 & 1.000 \\
$\varpi_{0}(437)$ & 1.000 & 0.960 & 1.000 \\
$\varpi_{0}(469)$ & 1.000 & 0.975 & 1.000 \\
$\varpi_{0}(502)$ & 1.000 & 0.980 & 1.000 \\
$\varpi_{0}(673)$ & 1.000 & 1.000 & 1.000
\end{tabular}

The initial model parameters. Units (if any) are indicated in brackets in the parameter column. The initial pressures for the tropospheric haze and sheet cloud are both fixed at 0.4 bar, 0.7 bar, and 1.0 bar. Thus, there are three initial models. 
Table 6. Optical Depth Retrievals

\begin{tabular}{ccccc}
\hline & Stratospheric Haze & Tropospheric Haze & Tropospheric Cloud \\
Region & $\tau_{1}(502)$ & $\tau_{2}(502)$ & $\begin{array}{c}\text { Correlation Coefficient } \\
\text { between } \tau_{2} \text { and } \tau_{3}\end{array}$ \\
\hline All Regions & $0.16 \pm 0.04$ & $4 . \pm 1$. & $30 . \pm 9$. & 0.147 \\
EZ & $0.22 \pm 0.02$ & $3.5 \pm 0.4$ & $29 . \pm 6$. & 0.418 \\
NEB & $0.10 \pm 0.06$ & $10 . \pm 6$. & $30 \pm 20$ & 0.974 \\
NTrZ & $0.21 \pm 0.04$ & $3.1 \pm 0.6$ & $35 . \pm 4$. & 0.048 \\
NTBs & $0.14 \pm 0.05$ & $4.6 \pm 0.2$ & $30 . \pm 5$. & 0.545 \\
NTBn & $0.17 \pm 0.04$ & $4.2 \pm 0.2$ & $31 . \pm 6$. & 0.818 \\
NTZ & $0.20 \pm 0.02$ & $4.2 \pm 0.3$ & $44 . \pm 5$. & -0.839 \\
GRS bright annulus & $0.17 \pm 0.03$ & $4.2 \pm 0.1$ & $27 . \pm 5$. & 0.583 \\
GRS center & $0.11 \pm 0.04$ & $4.8 \pm 0.4$ & $28 . \pm 6$. & 0.789 \\
GRS red annulus & $0.16 \pm 0.04$ & $4.2 \pm 0.2$ & $26 . \pm 4$. & 0.327 \\
BA center & $0.17 \pm 0.03$ & $3.8 \pm 0.4$ & $41 . \pm 5$. & -0.041 \\
BA red annulus & $0.20 \pm 0.02$ & $4.3 \pm 0.2$ & $40 . \pm 7$. & 0.421 \\
Oval 2 & $0.15 \pm 0.03$ & $4.4 \pm 0.2$ & $26 . \pm 3$. & -0.005 \\
NEB1 & $0.10 \pm 0.04$ & $9 . \pm \pm$. & $30 \pm 20$ & 0.995 \\
A5 & $0.13 \pm 0.02$ & $3.3 \pm 0.5$ & $46 . \pm 3$. & 0.541 \\
\hline
\end{tabular}


Table 7. Single Scattering Albedo Retrievals

\begin{tabular}{|c|c|c|c|c|c|c|c|c|c|}
\hline Region & $\varpi_{0}(255)^{a}$ & $\omega_{0}(343)^{\alpha}$ & $\varpi_{0}(375)$ & $\varpi_{0}(390)$ & $\varpi_{0}(410)$ & $\varpi_{0}(437)$ & $\varpi_{0}(469)$ & $\varpi_{0}(502)$ & $\varpi_{0}(673)$ \\
\hline All Regions & $0.873 \pm 0.025$ & $0.893 \pm 0.029$ & $0.902 \pm 0.016$ & $0.924 \pm 0.013$ & $0.946 \pm 0.010$ & $0.962 \pm 0.009$ & $0.975 \pm 0.007$ & $0.984 \pm 0.005$ & $0.999 \pm 0.001$ \\
\hline EZ & $0.865 \pm 0.015$ & $0.916 \pm 0.009$ & $0.922 \pm 0.004$ & $0.943 \pm 0.003$ & $0.960 \pm 0.002$ & $0.972 \pm 0.002$ & $0.980 \pm 0.002$ & $0.986 \pm 0.001$ & $0.998 \pm 0.001$ \\
\hline NEB & $0.891 \pm 0.027$ & $0.878 \pm 0.036$ & $0.904 \pm 0.029$ & $0.916 \pm 0.021$ & $0.936 \pm 0.015$ & $0.947 \pm 0.010$ & $0.960 \pm 0.007$ & $0.971 \pm 0.005$ & $0.996 \pm 0.003$ \\
\hline NTrZ & $0.878 \pm 0.011$ & $0.933 \pm 0.011$ & $0.931 \pm 0.009$ & $0.948 \pm 0.006$ & $0.963 \pm 0.003$ & $0.973 \pm 0.002$ & $0.981 \pm 0.001$ & $0.986 \pm 0.001$ & $0.999 \pm 0.001$ \\
\hline NTBs & $0.863 \pm 0.032$ & $0.883 \pm 0.033$ & $0.888 \pm 0.016$ & $0.909 \pm 0.011$ & $0.933 \pm 0.007$ & $0.950 \pm 0.005$ & $0.967 \pm 0.004$ & $0.978 \pm 0.002$ & $1.000 \pm 0.000$ \\
\hline NTBn & $0.878 \pm 0.017$ & $0.891 \pm 0.009$ & $0.897 \pm 0.008$ & $0.919 \pm 0.006$ & $0.942 \pm 0.004$ & $0.959 \pm 0.003$ & $0.974 \pm 0.002$ & $0.983 \pm 0.002$ & $1.000 \pm 0.001$ \\
\hline NTZ & $0.859 \pm 0.013$ & $0.907 \pm 0.005$ & $0.913 \pm 0.008$ & $0.938 \pm 0.005$ & $0.958 \pm 0.003$ & $0.972 \pm 0.002$ & $0.983 \pm 0.001$ & $0.989 \pm 0.001$ & $1.000 \pm 0.000$ \\
\hline GRS bright annulus & $0.863 \pm 0.012$ & $0.883 \pm 0.020$ & $0.897 \pm 0.008$ & $0.922 \pm 0.006$ & $0.946 \pm 0.005$ & $0.962 \pm 0.004$ & $0.975 \pm 0.003$ & $0.984 \pm 0.002$ & $0.999 \pm 0.001$ \\
\hline GRS center & $0.842 \pm 0.025$ & $0.860 \pm 0.028$ & $0.893 \pm 0.020$ & $0.913 \pm 0.014$ & $0.936 \pm 0.009$ & $0.952 \pm 0.006$ & $0.967 \pm 0.004$ & $0.978 \pm 0.003$ & $0.999 \pm 0.001$ \\
\hline GRS red annulus & $0.878 \pm 0.017$ & $0.889 \pm 0.032$ & $0.899 \pm 0.011$ & $0.919 \pm 0.009$ & $0.942 \pm 0.006$ & $0.958 \pm 0.005$ & $0.973 \pm 0.003$ & $0.982 \pm 0.002$ & $0.999 \pm 0.001$ \\
\hline BA center & $0.874 \pm 0.010$ & $0.915 \pm 0.011$ & $0.925 \pm 0.008$ & $0.945 \pm 0.005$ & $0.962 \pm 0.003$ & $0.974 \pm 0.002$ & $0.984 \pm 0.001$ & $0.990 \pm 0.001$ & $0.999 \pm 0.001$ \\
\hline BA red annulus & $0.903 \pm 0.013$ & $0.922 \pm 0.015$ & $0.910 \pm 0.007$ & $0.931 \pm 0.005$ & $0.953 \pm 0.003$ & $0.967 \pm 0.003$ & $0.979 \pm 0.001$ & $0.987 \pm 0.001$ & $1.000 \pm 0.000$ \\
\hline Oval 2 & $0.858 \pm 0.025$ & $0.884 \pm 0.020$ & $0.889 \pm 0.009$ & $0.913 \pm 0.006$ & $0.938 \pm 0.005$ & $0.956 \pm 0.004$ & $0.971 \pm 0.002$ & $0.981 \pm 0.002$ & $0.999 \pm 0.001$ \\
\hline NEB1 & $0.940 \pm 0.012$ & $0.924 \pm 0.034$ & $0.896 \pm 0.023$ & $0.902 \pm 0.021$ & $0.922 \pm 0.017$ & $0.936 \pm 0.010$ & $0.953 \pm 0.007$ & $0.964 \pm 0.007$ & $0.995 \pm 0.002$ \\
\hline A5 & $0.893 \pm 0.006$ & $0.928 \pm 0.012$ & $0.944 \pm 0.008$ & $0.958 \pm 0.006$ & $0.969 \pm 0.005$ & $0.978 \pm 0.004$ & $0.985 \pm 0.002$ & $0.990 \pm 0.001$ & $1.000 \pm 0.000$ \\
\hline
\end{tabular}

\footnotetext{
${ }^{a}$ The values reported here for $\varpi_{0}(255)$ and $\varpi_{0}(343)$ are for stratospheric haze particles. $\varpi_{0}(255)$ and $\varpi_{0}(343)$ for tropospheric haze particles are equal to $\varpi_{0}(375)$.
} 
Table 8. Best-fit Parameters for the Empirical Functional Form for $\varpi_{0}(\lambda)$

\begin{tabular}{ccc}
\hline & Parameter & Parameter \\
Region & A & B \\
\hline All Regions & $34.1 \pm 18.8$ & $0.0151 \pm 0.0020$ \\
EZ & $28.0 \pm 8.0$ & $0.0157 \pm 0.0009$ \\
NEB & $4.0 \pm 2.9$ & $0.0093 \pm 0.0015$ \\
NTrZ & $17.4 \pm 10.4$ & $0.0145 \pm 0.0011$ \\
NTBs & $17.2 \pm 8.8$ & $0.0131 \pm 0.0013$ \\
NTBn & $31.7 \pm 12.1$ & $0.0150 \pm 0.0014$ \\
NTZ & $79.1 \pm 18.9$ & $0.0182 \pm 0.0005$ \\
GRS bright annulus & $39.7 \pm 13.4$ & $0.0157 \pm 0.0012$ \\
GRS center & $16.6 \pm 12.2$ & $0.0129 \pm 0.0015$ \\
GRS red annulus & $22.6 \pm 8.3$ & $0.0142 \pm 0.0011$ \\
BA center & $47.0 \pm 22.1$ & $0.0170 \pm 0.0010$ \\
BA red annulus & $44.7 \pm 19.5$ & $0.0164 \pm 0.0009$ \\
Oval 2 & $31.6 \pm 13.1$ & $0.0149 \pm 0.0010$ \\
NEB1 & $3.2 \pm 1.8$ & $0.0086 \pm 0.0012$ \\
A5 & $13.3 \pm 2.5$ & $0.0146 \pm 0.0003$
\end{tabular}

The functional form (Eq. 7 ) is $\varpi_{0}(\lambda)=1-A \exp (-B \lambda)$ for $\lambda$ in units of $\mathrm{nm}$. 


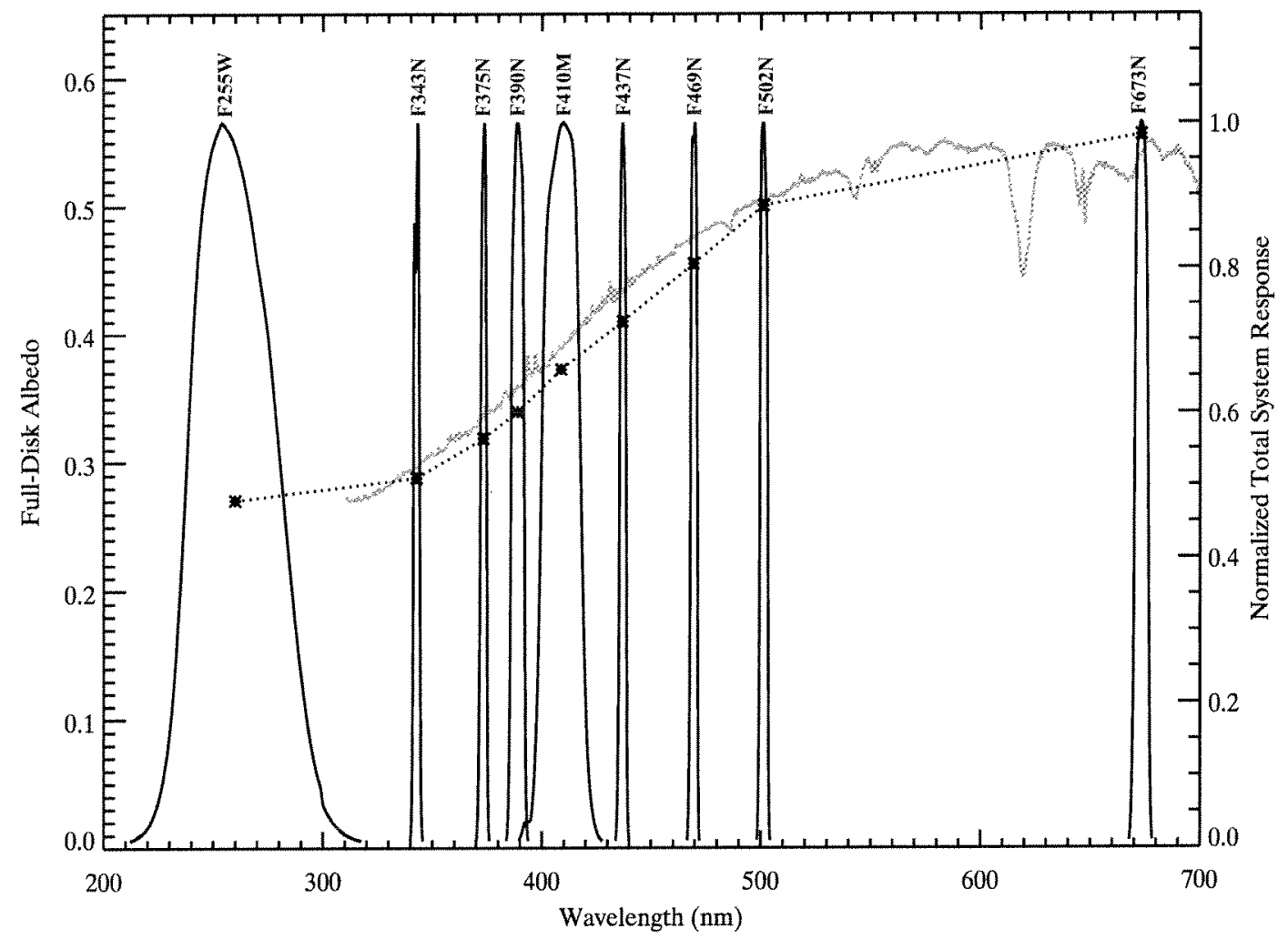

Fig. 1. Throughput curves for HST WFPC2 filters (solid black) used in the observations, the approximate full-disk albedo spectrum of the data (dotted black) with the corrected value for F343N, and the reference full-disk albedo spectrum of Jupiter (gray) from Karkoschka (1998). 


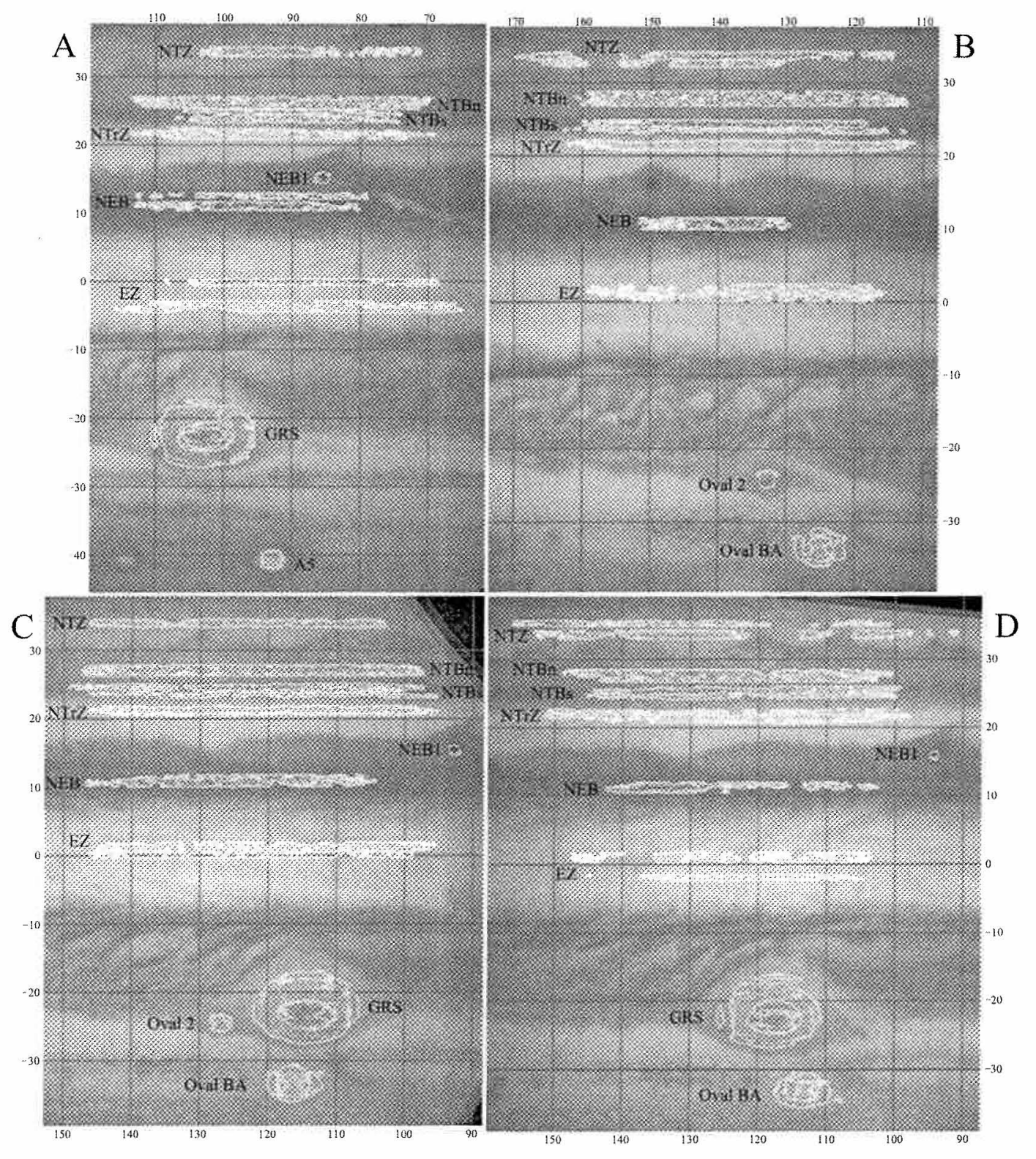

Fig. 2. All four mapped data cubes. Cubes A and B are from 15 May 2008, Cube C is from 28 June 2008, and Cube D is from 08 July 2008. Locations that are modeled in this work are outlined in white. Latitudes are planetographic, and longitudes are System III. Colors are approximately true-color. Each color channel is the average of the $I / F$ values in the given filters and scaled from 0 to 1 : Red $=502-673 \mathrm{~nm}$, Green $=437-469 \mathrm{~nm}$, Blue $=255-410 \mathrm{~nm}$. No limb-darkening correction was applied, which can be seen most readily on the left sides of $\mathrm{B}$ and $\mathrm{D}$. These are greenish where $\mu_{0}$ is low in the blue channel images. 


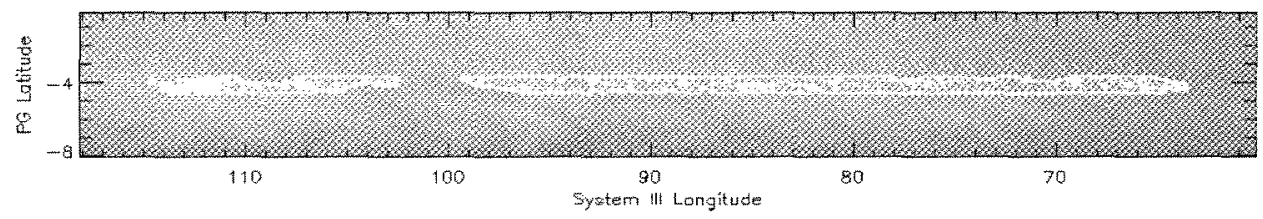

Fig. 3. An example data cut (areas outlined in white) selected from the EZ in Cube A is shown with the same color channels as in Fig. 2. The samples in the data cut that were selected for modeling are the averages of pixels within $1^{\circ}$ bins in longitude, which are the size of the minor tick marks in this figure.

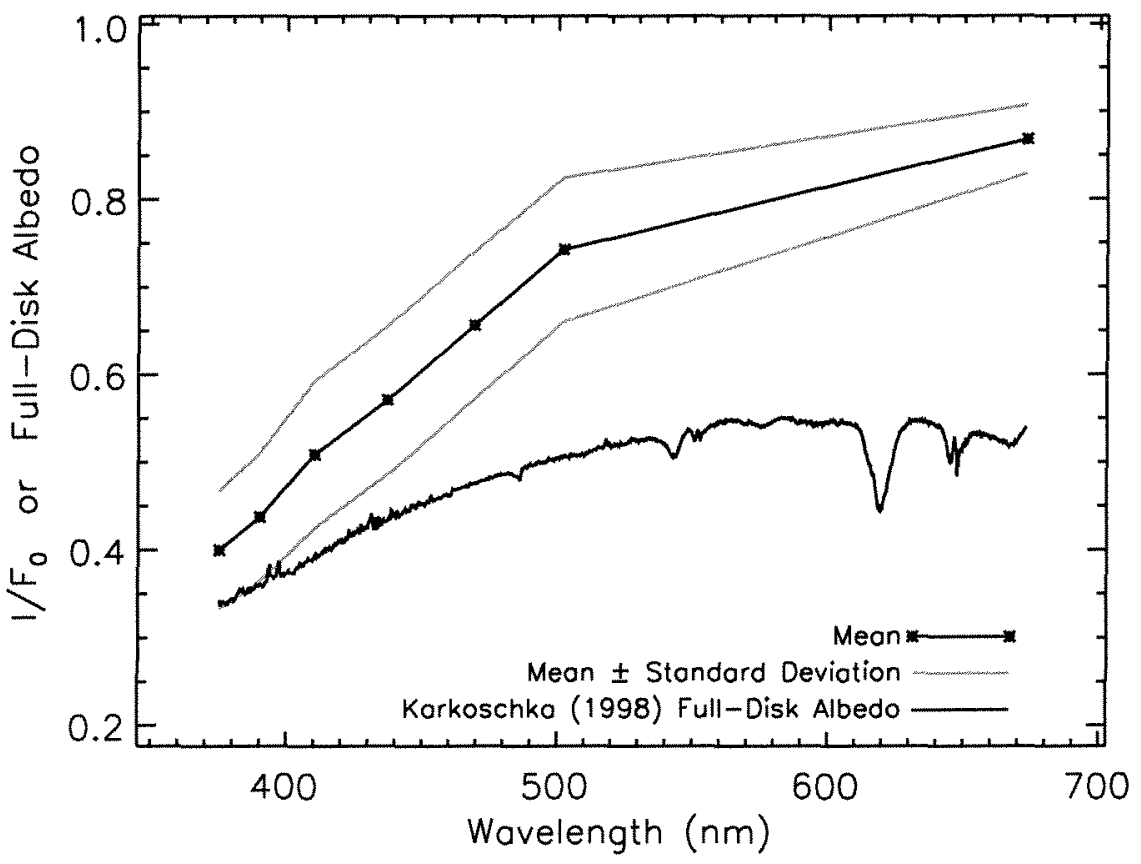

Fig. 4. The mean and standard deviation of the $I / F_{0}$ spectra for all 1345 data cuts. The reference full-disk albedo spectrum from Karkoschka (1998) lies below the $I / F_{0}$ spectra because it does not account for limb darkening. 

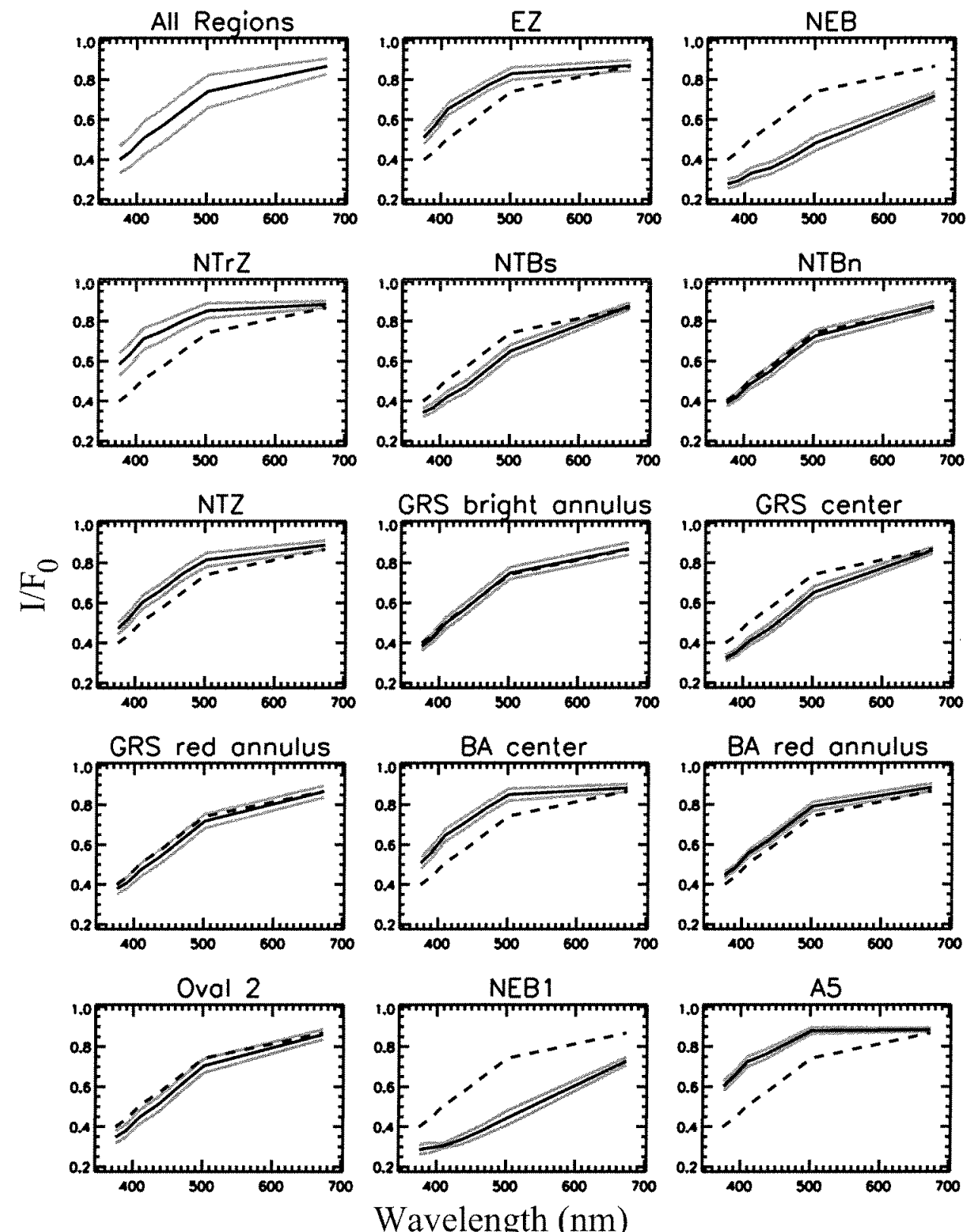

Wavelength $(\mathrm{nm})$

Fig. 5. The mean (solid black) and standard deviation (gray) of $I / F_{0}(\lambda)$ for all data cuts separated by weather region. The dashed black line is the mean for all data cuts from all weather regions. All panels have the same abscissa and ordinate ranges. 

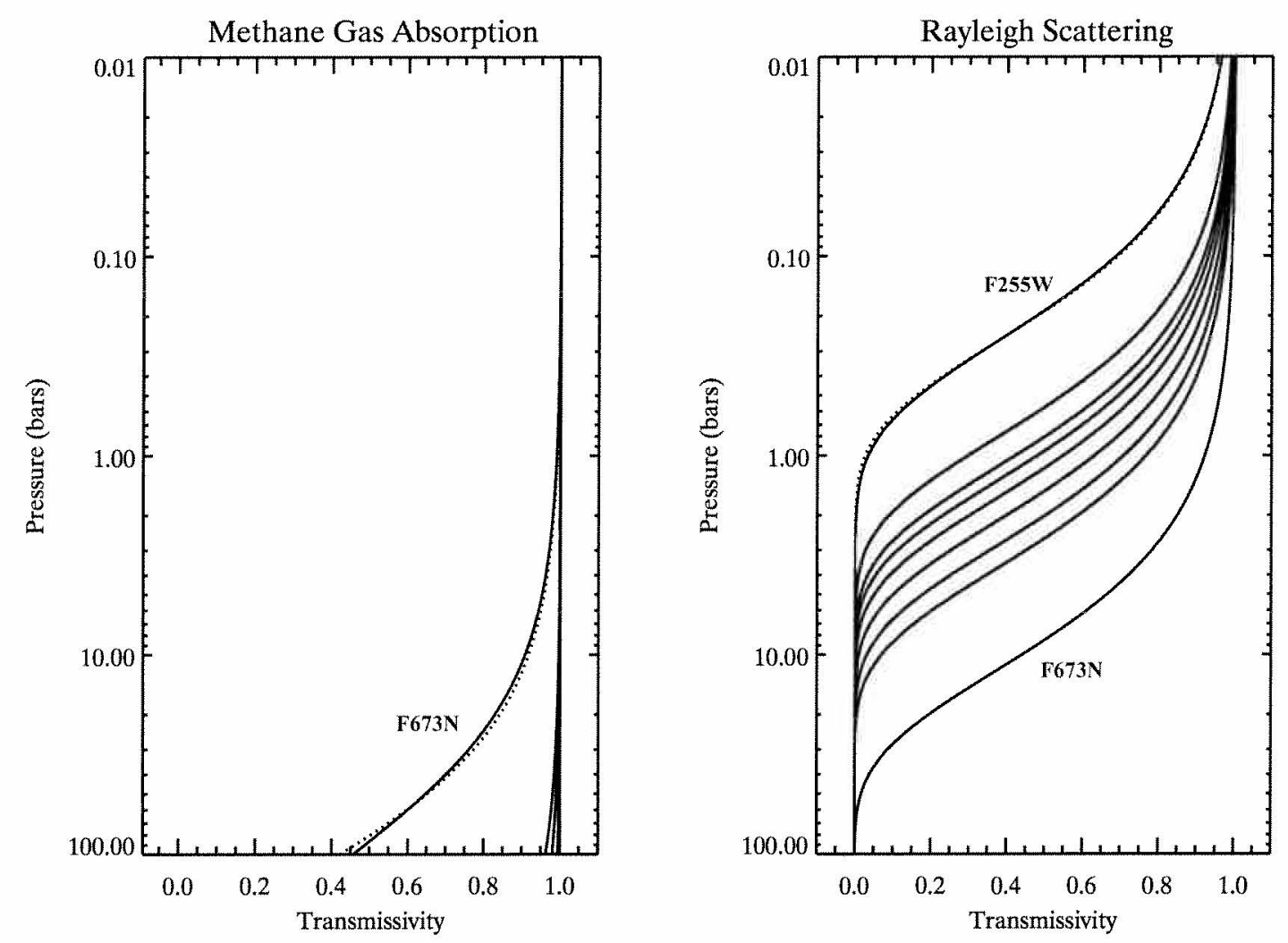

Fig. 6. Two-way transmissivities for HST WFPC2 filters (solid curves) assuming a nadir view and no aerosol opacity. The left panel contains gas absorption transmissivities calculated from the Karkoschka (1998) methane absorption coefficients. Curves for all but the $\mathrm{F} 673 \mathrm{~N}$ filter are overlapping at a transmissivity value of unity from space down to $\sim 10$ bars. The right panel contains two-way transmissivities for Rayleigh scattering. The curves progress from $\mathrm{F} 255 \mathrm{~W}$ to $\mathrm{F} 673 \mathrm{~N}$ in order of wavelength. The dotted curves in both plots are the Beer's Law fits used in the radiative transfer code (see Table 3). Most fits coincide with the actual transmissivities at this resolution. 


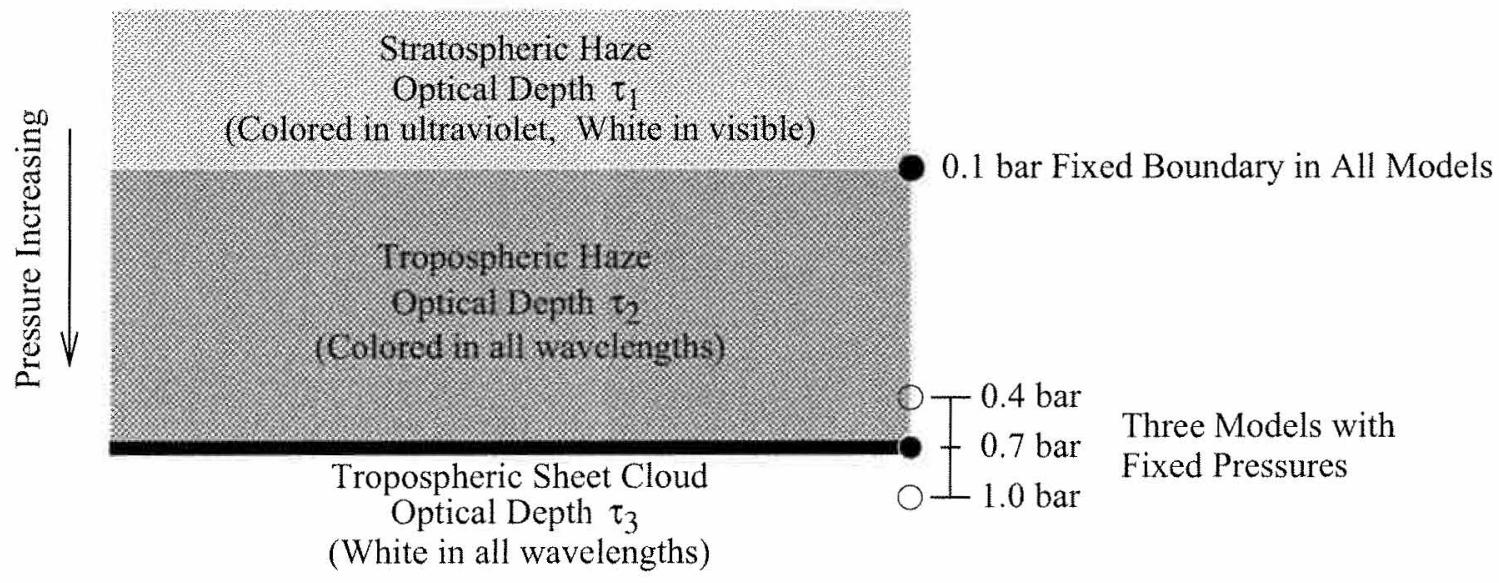

Fig. 7. Schematic of the vertical structure models used in this work. 

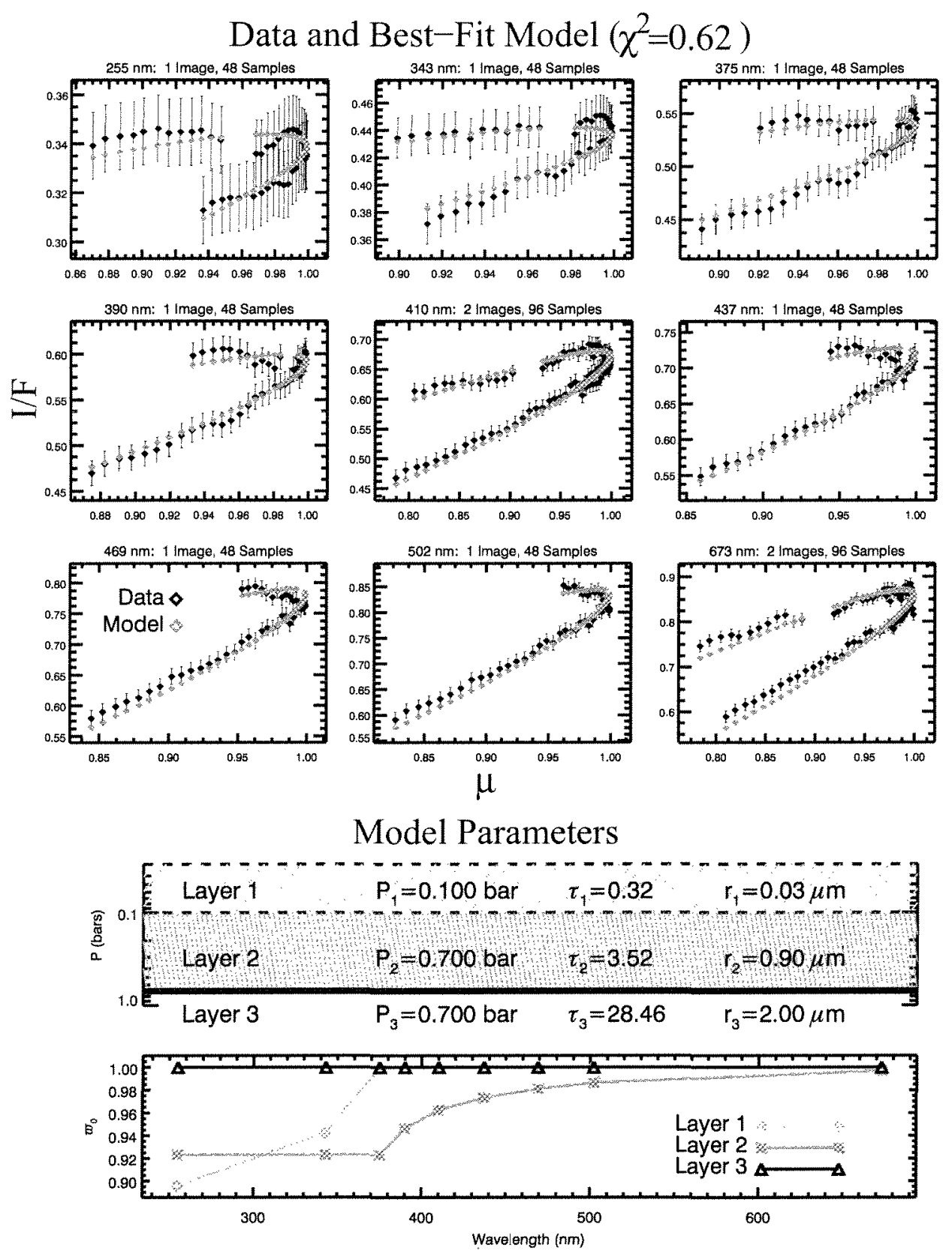

Fig. 8. Example data cut and best-fit model using the $\chi^{2}$-minimization routine (see Fig. 9). The data cut (Fig. 3, outlined areas) is from the EZ in Cube A. The samples in the data cut that were selected for modeling (black points in top plots) are the averages of pixels within $1^{\circ}$ bins in longitude. The wavelength, and the number of images and samples at that wavelength, are noted above each plot of $I / F$ versus $\mu$. The error bars are the estimated errors used to calculate $\chi^{2}$. The green points are the radiative transfer results generated by the model parameters at the bottom of the figure. 

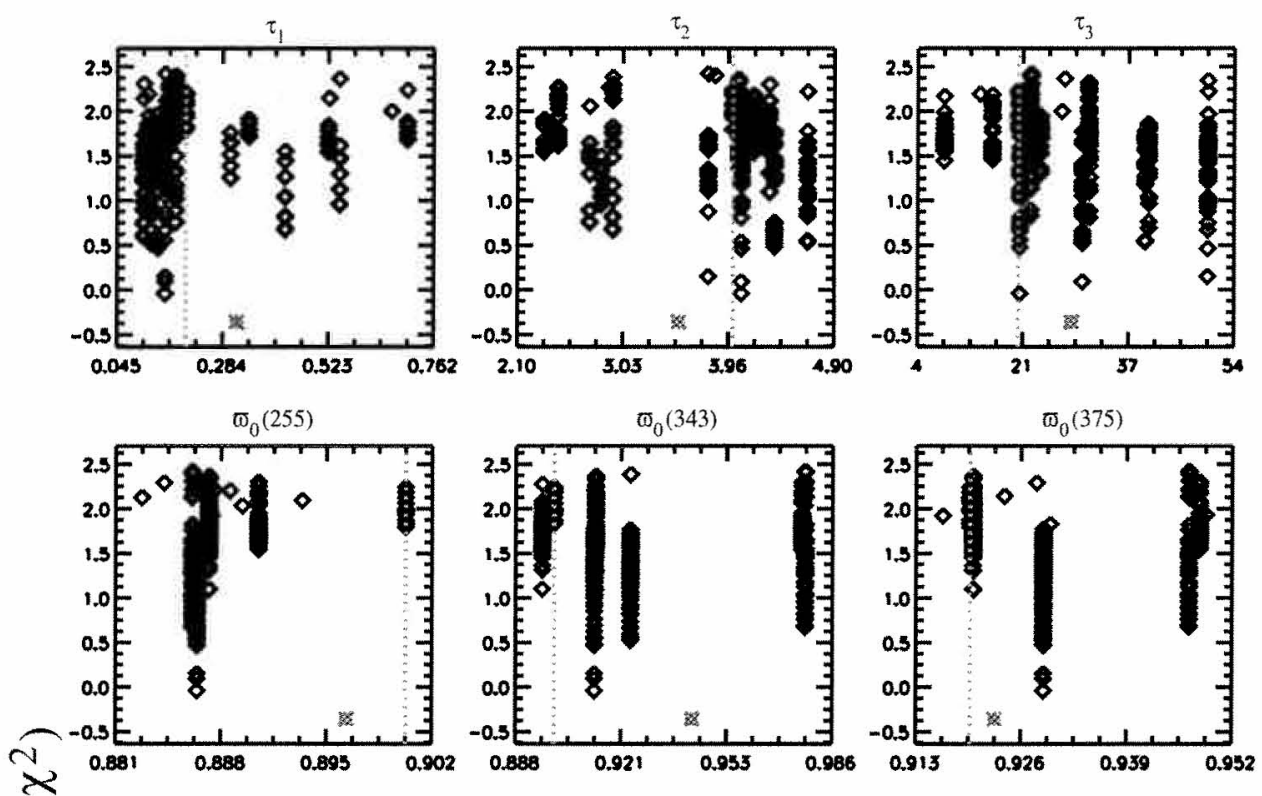

$\frac{x}{0}$
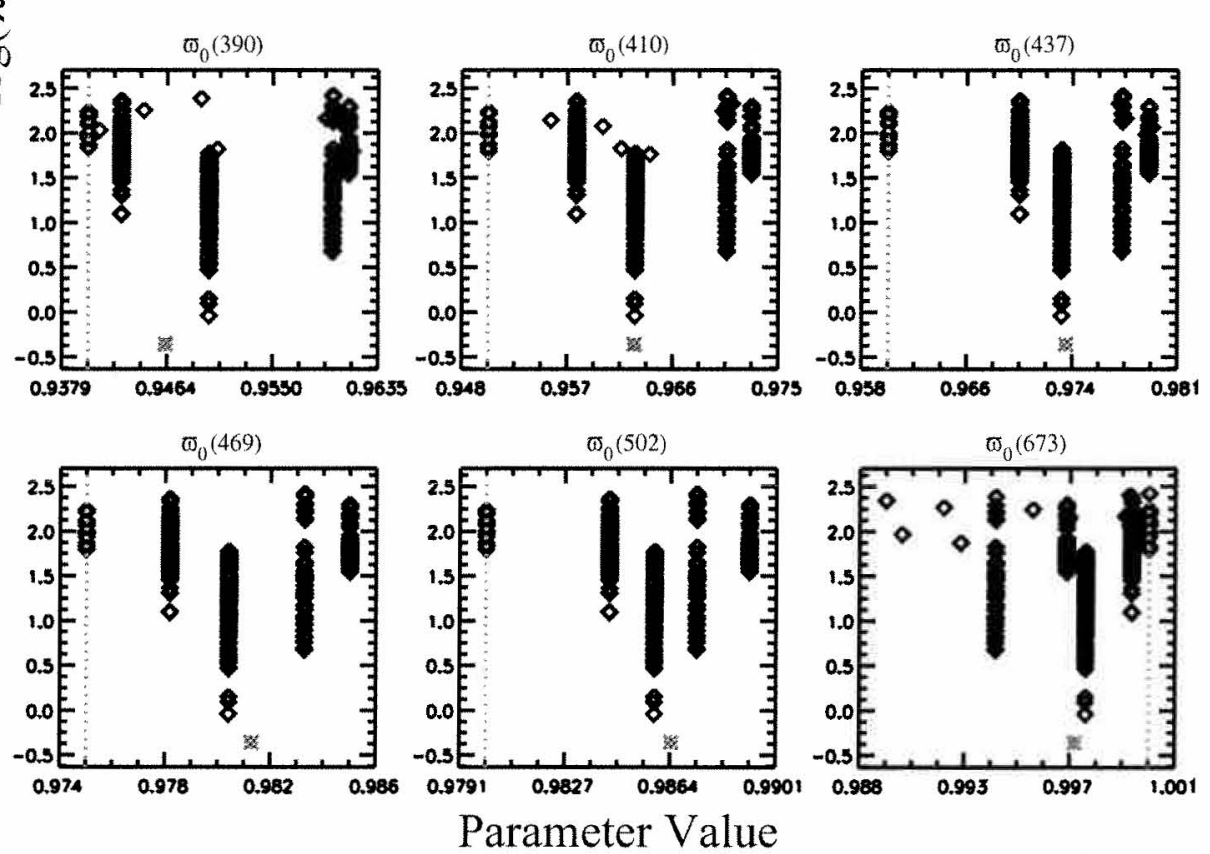

Fig. 9. Example convergence of parameters using the $\chi^{2}$-minimization routine. The data cut (Fig. 3, outlined areas) is from the EZ in Cube A. The diamonds are from models saved during Phases A and B, and the asterisks are the best-fit model at the completion of Phase C (see Fig. 8). The dotted lines mark the initial parameter value (Table 5). 

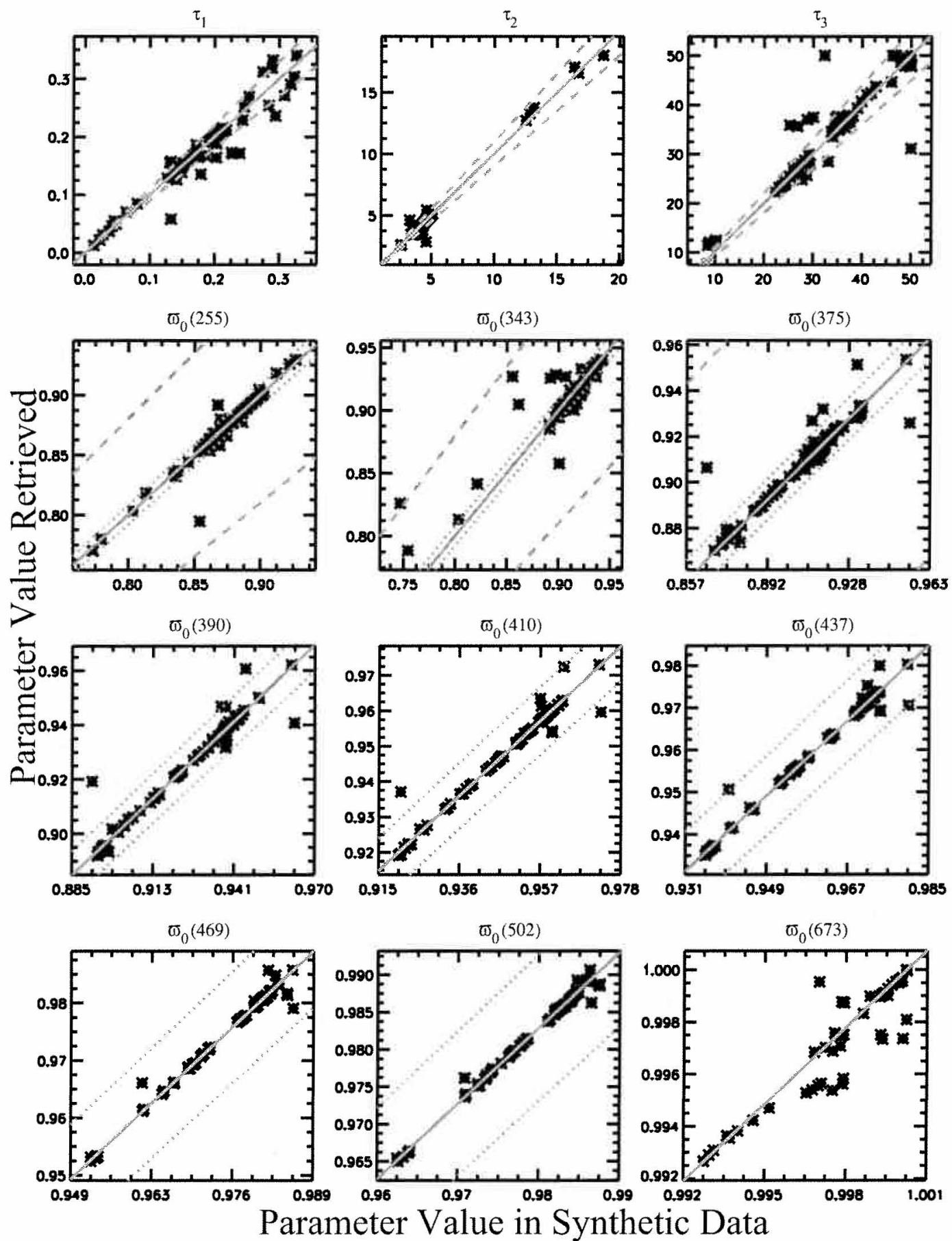

Fig. 10. Retrievals from synthetic data with added Gaussian noise $(\sigma=0.005[I / F])$.

The difference between the parameter values used to generate the synthetic data and those retrieved are marked at values of $0 \%$ (solid line), $1 \%$ (dotted line), and $10 \%$ (dashed line). 

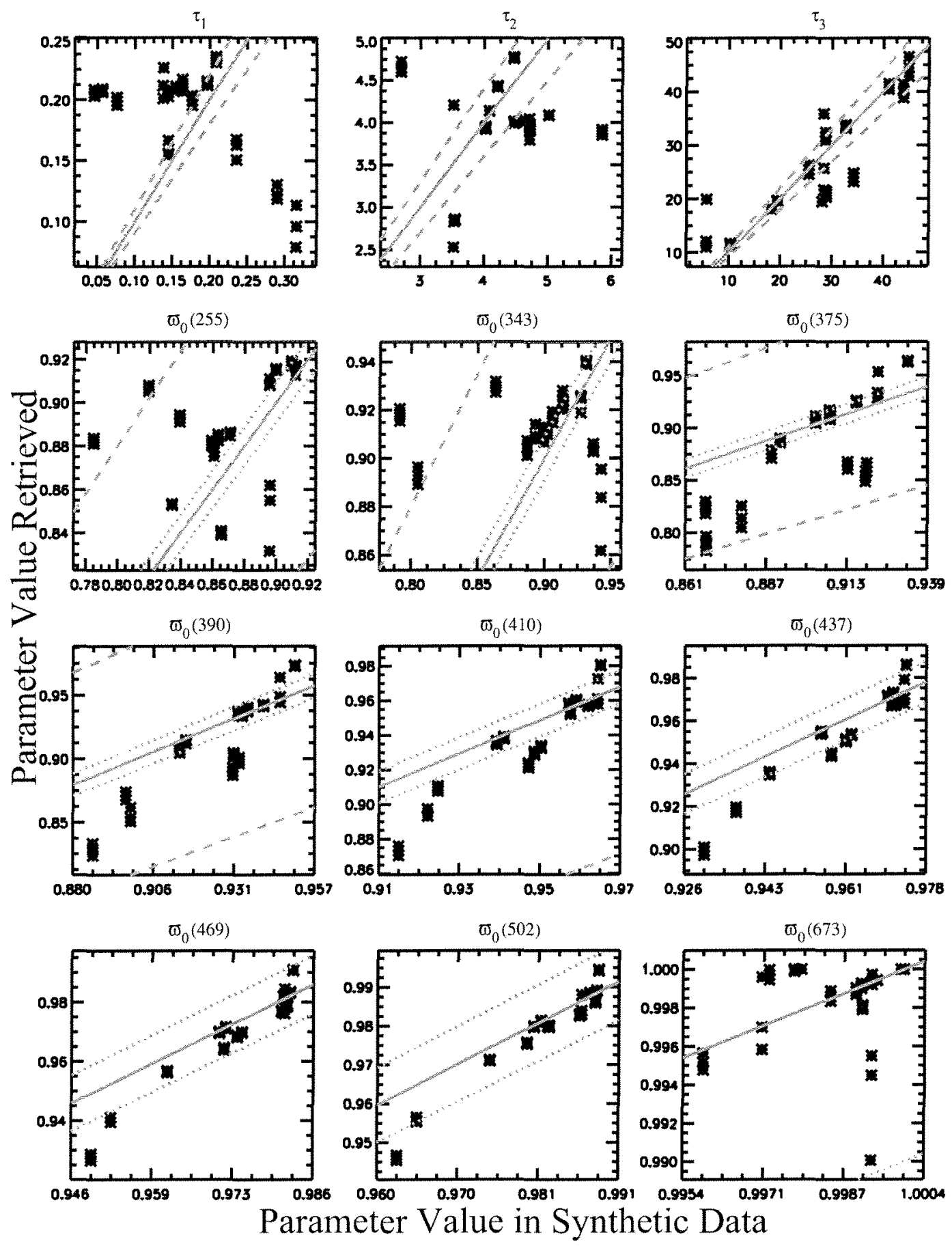

Fig. 11. Retrievals from synthetic data with the stratospheric/tropospheric haze interface $\left(P_{1}\right)$ pressure set to 0.2 bar. The retrievals assumed $P_{1}=0.1$ bar. The difference between the true parameter values and those retrieved are marked at values of $0 \%$ (solid line), $1 \%$ (dotted line), and 10\% (dashed line). 

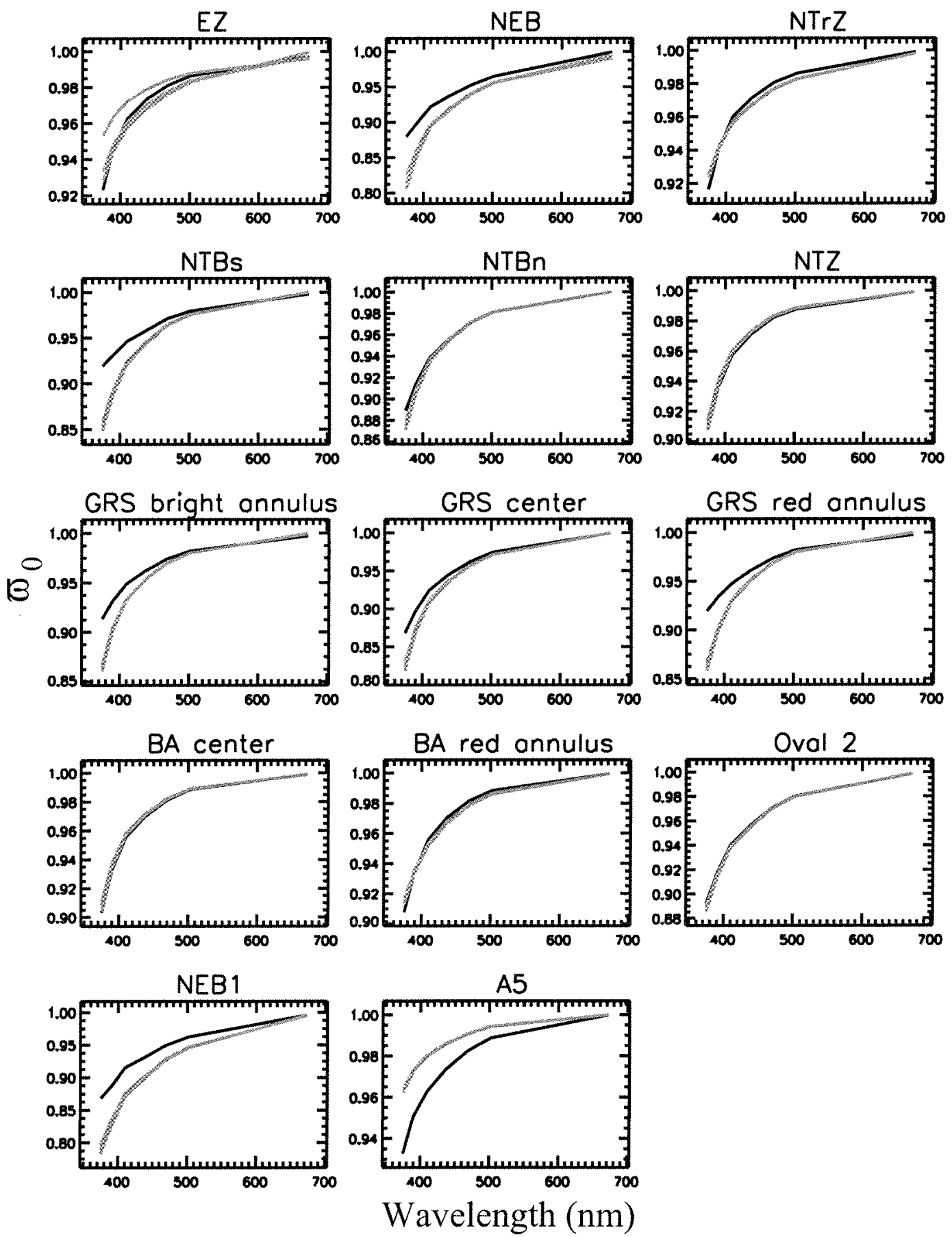

Fig. 12. Retrievals from synthetic data with the stratospheric/tropospheric haze interface $\left(P_{1}\right)$ pressure set to 0.2 bar. The retrievals assumed $P_{1}=0.1$ bar. The solid lines are the $\varpi_{0}(\lambda)$ values of the synthetic data, and the gray lines are the retrievals. There are three models $\left(P_{3}=[0.4,0.7,1.0]\right.$ bar $)$, and all three were used for these retrievals. In most of these plots the three gray lines overlap, because all three models usually converged to similar $\varpi_{0}(\lambda)$ curves. 

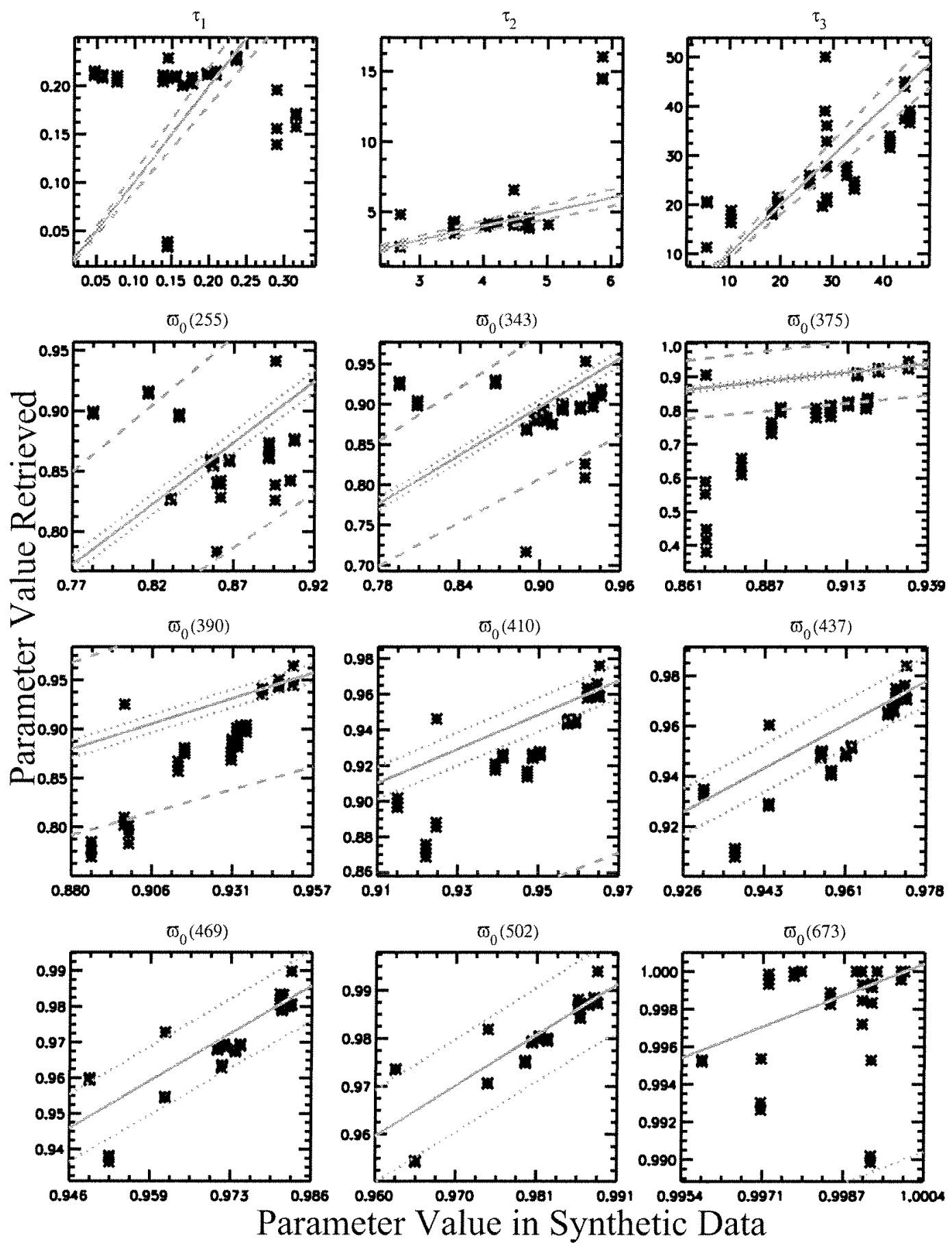

Fig. 13. The same as Fig. 11, except for retrievals from synthetic data with the radius of stratospheric haze particles set to $0.1 \mu \mathrm{m}$. The retrievals assumed $0.03 \mu \mathrm{m}$ particles. 
$E Z$
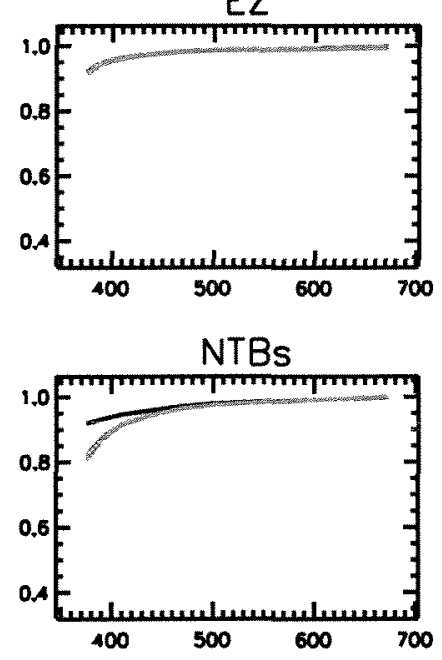

GRS bright onnulus

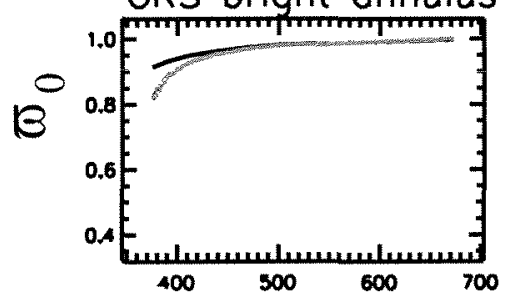

BA center

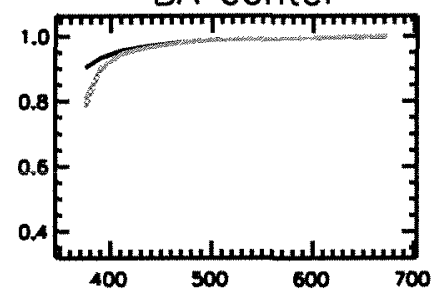

NEB 1

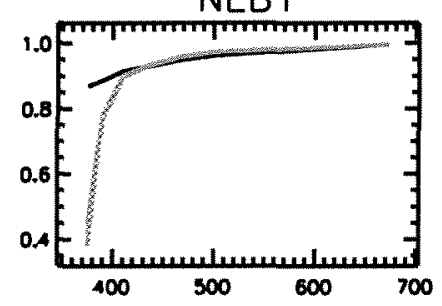

NEB

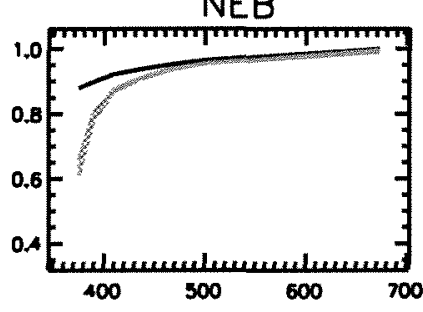

NTBn

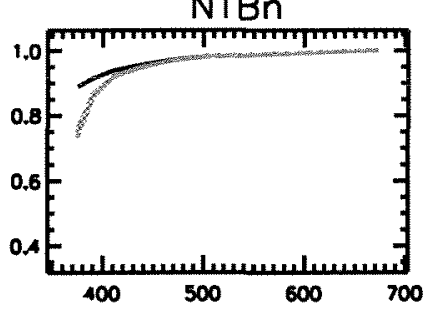

GRS center

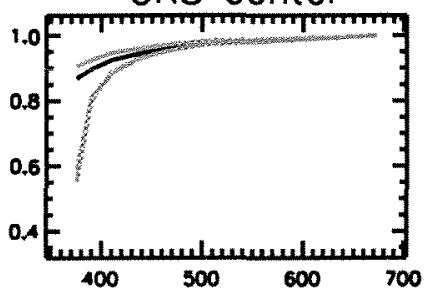

BA red annulus

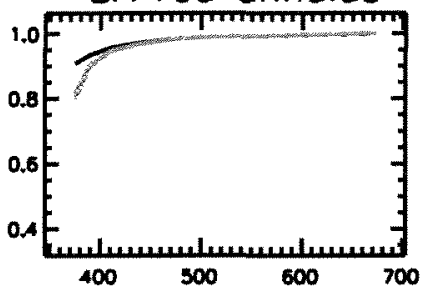

A5

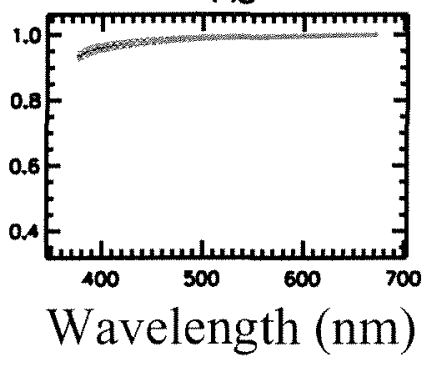

NTrZ

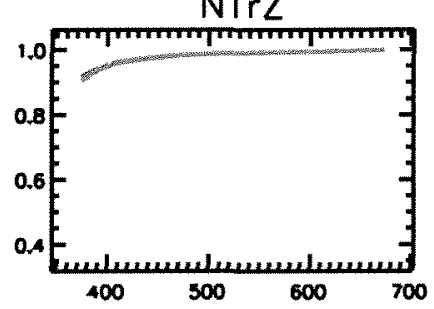

NTZ

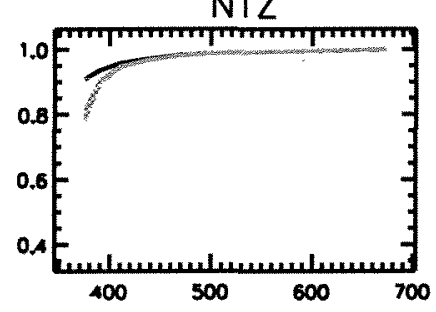

GRS red annulus

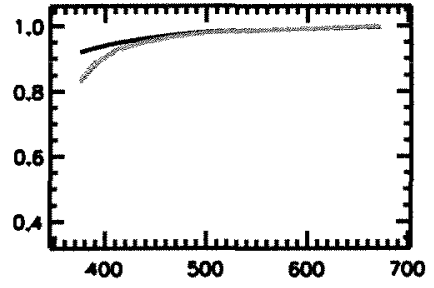

Oval 2

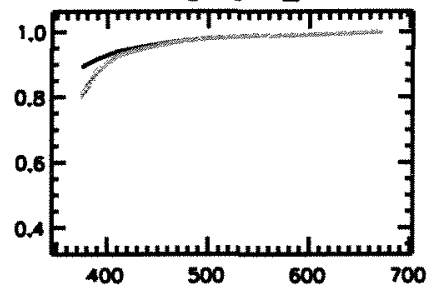

Fig. 14. The same as Fig. 12, except for retrievals from synthetic data with the radius of stratospheric haze particles set to $0.1 \mu \mathrm{m}$. The retrievals assumed $0.03 \mu \mathrm{m}$ particles. 

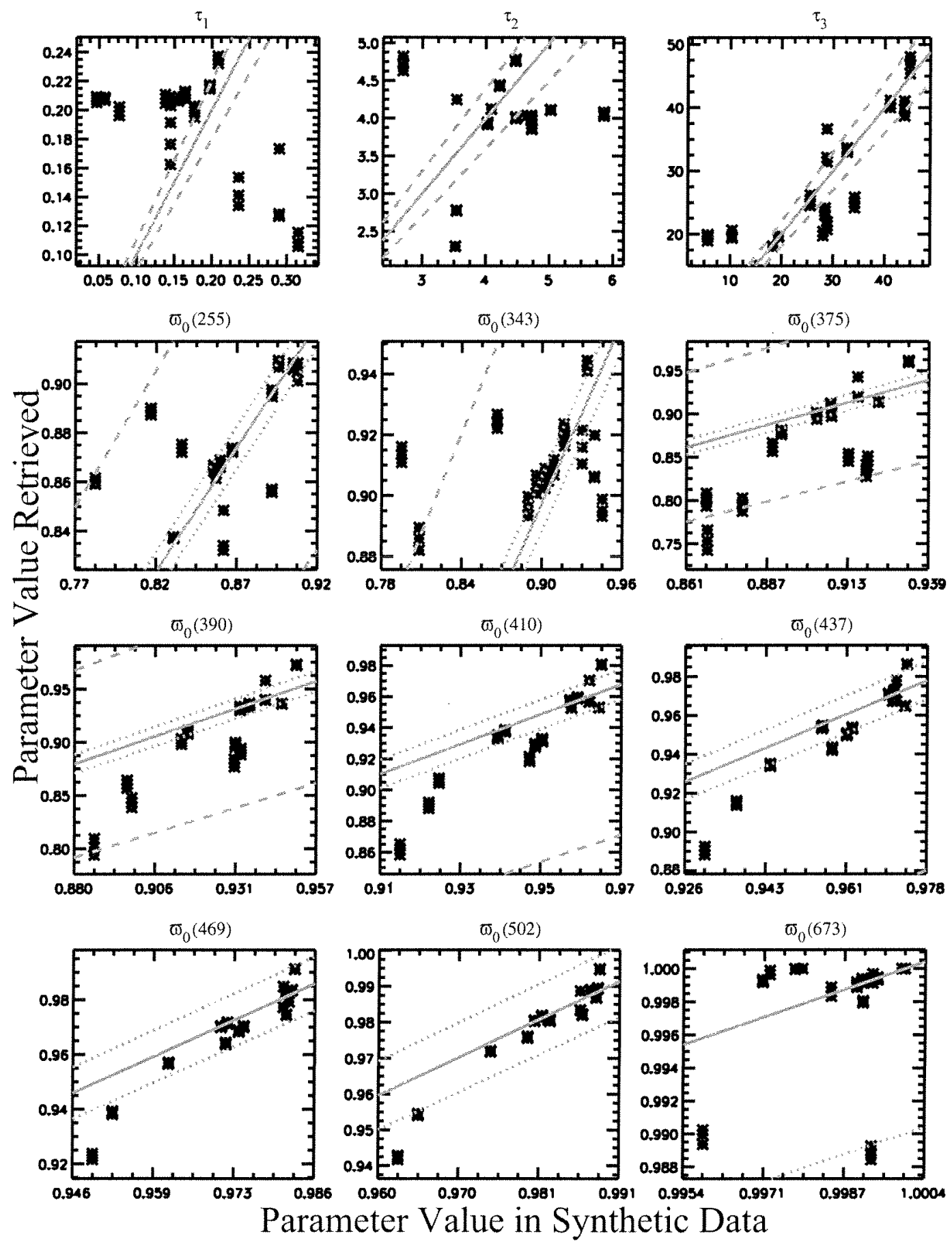

Fig. 15. The same as Fig. 11, except for retrievals from synthetic data with the radius of tropospheric haze particles set to $2.0 \mu \mathrm{m}$. The retrievals assumed $0.9 \mu \mathrm{m}$ particles. 
$E Z$

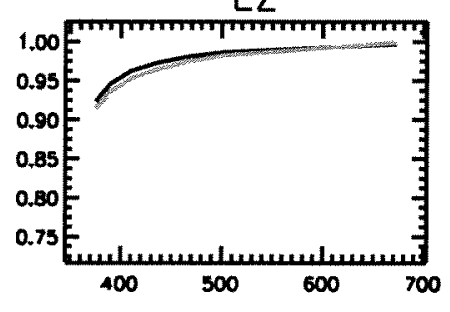

NTBs

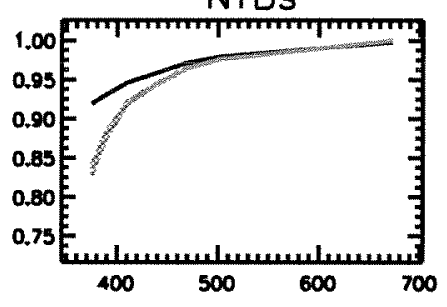

GRS bright annulus

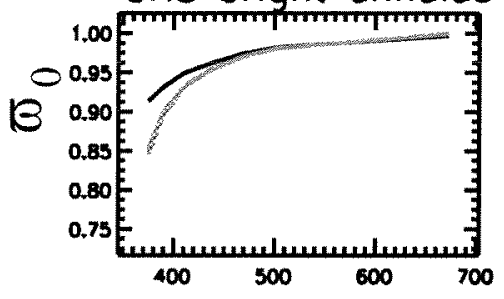

BA center

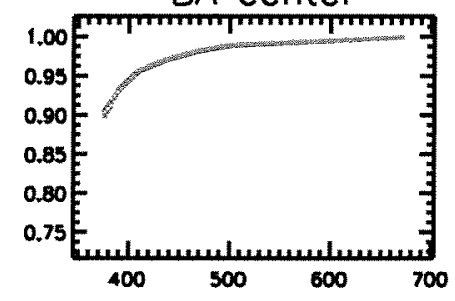

NEB 1

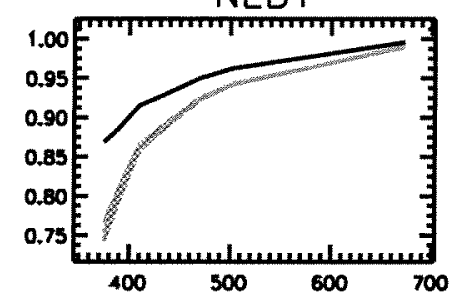

NEB
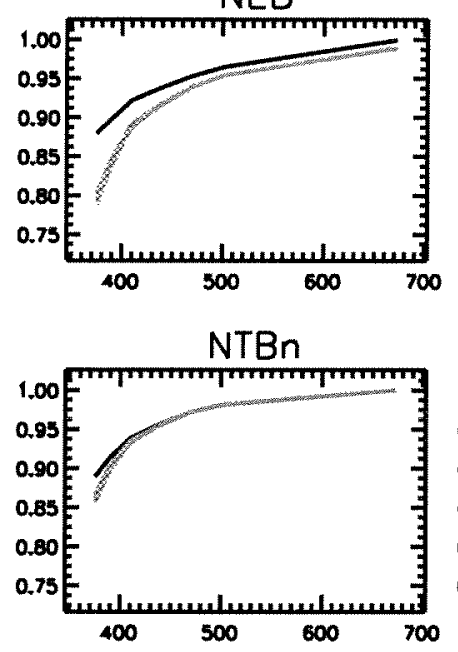

GRS center

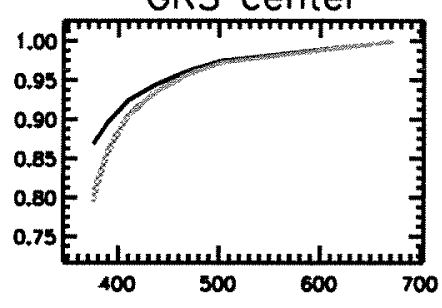

$B A$ red annulus

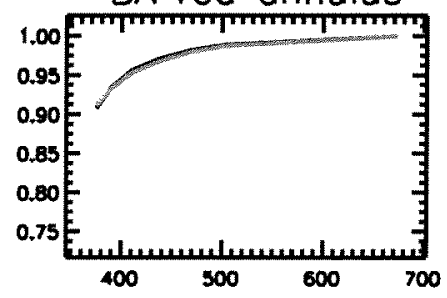

A5

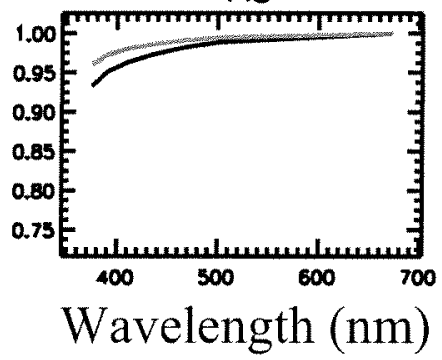

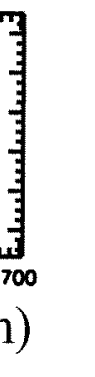
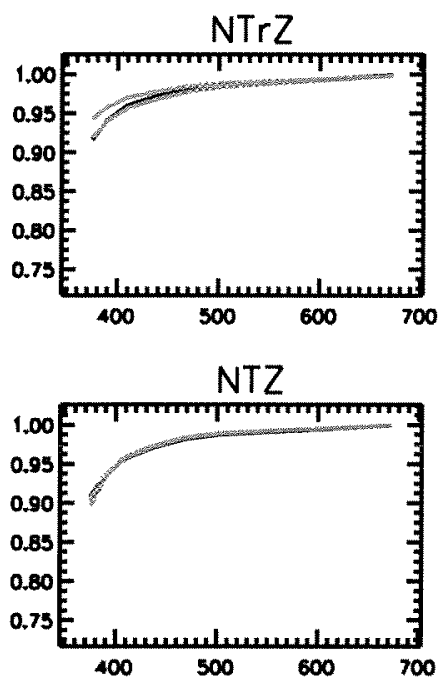

GRS red annulus

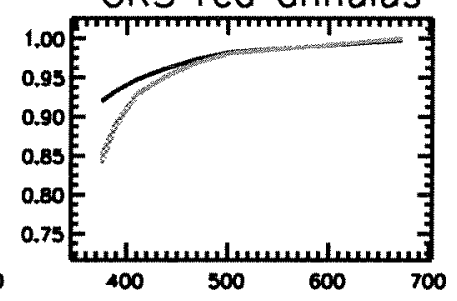

Oval 2

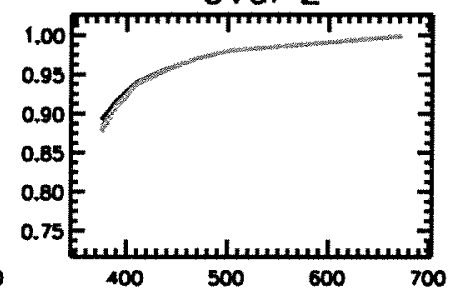

Fig. 16. The same as Fig. 12, except for retrievals from synthetic data with the radius of tropospheric haze particles set to $2.0 \mu \mathrm{m}$. The retrievals assumed $0.9 \mu \mathrm{m}$ particles. 

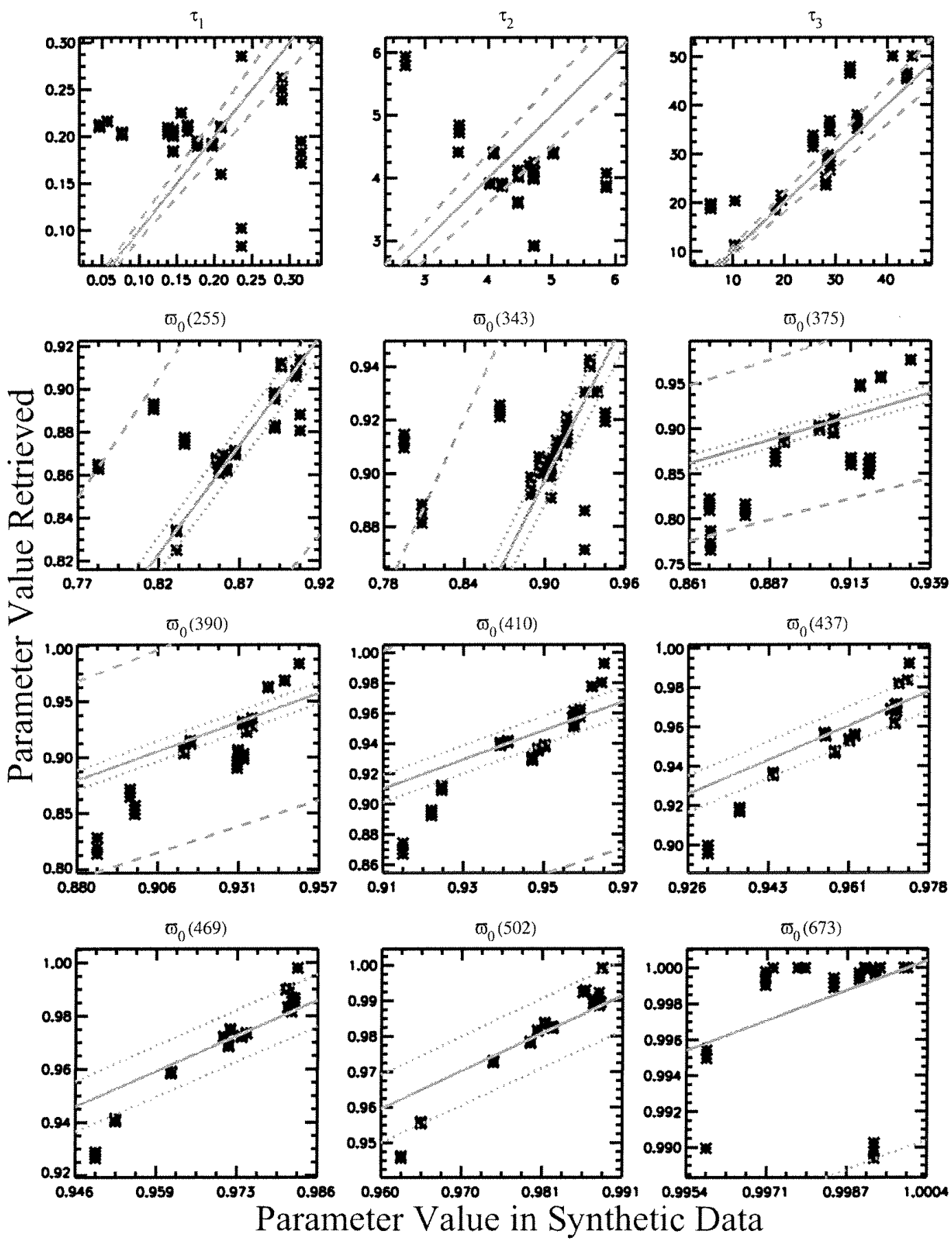

Fig. 17. The same as Fig. 11, except for retrievals from synthetic data with the radius of tropospheric cloud particles set to $4.0 \mu \mathrm{m}$. The retrievals assumed $2.0 \mu \mathrm{m}$ particles. 
$E Z$

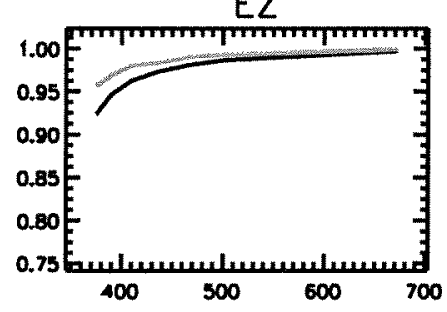

NTBs

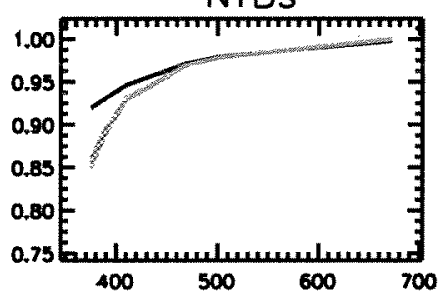

GRS bright annulus

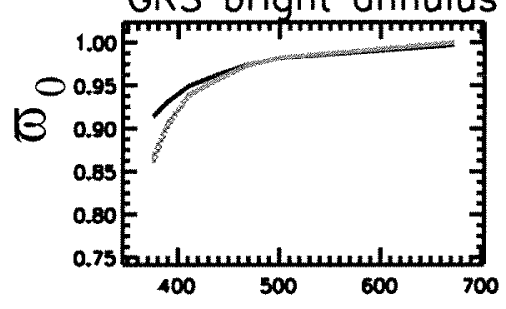

BA center

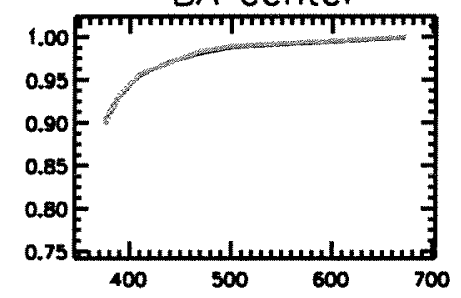

NEB1

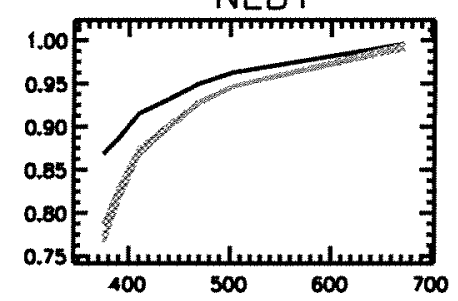

NEB
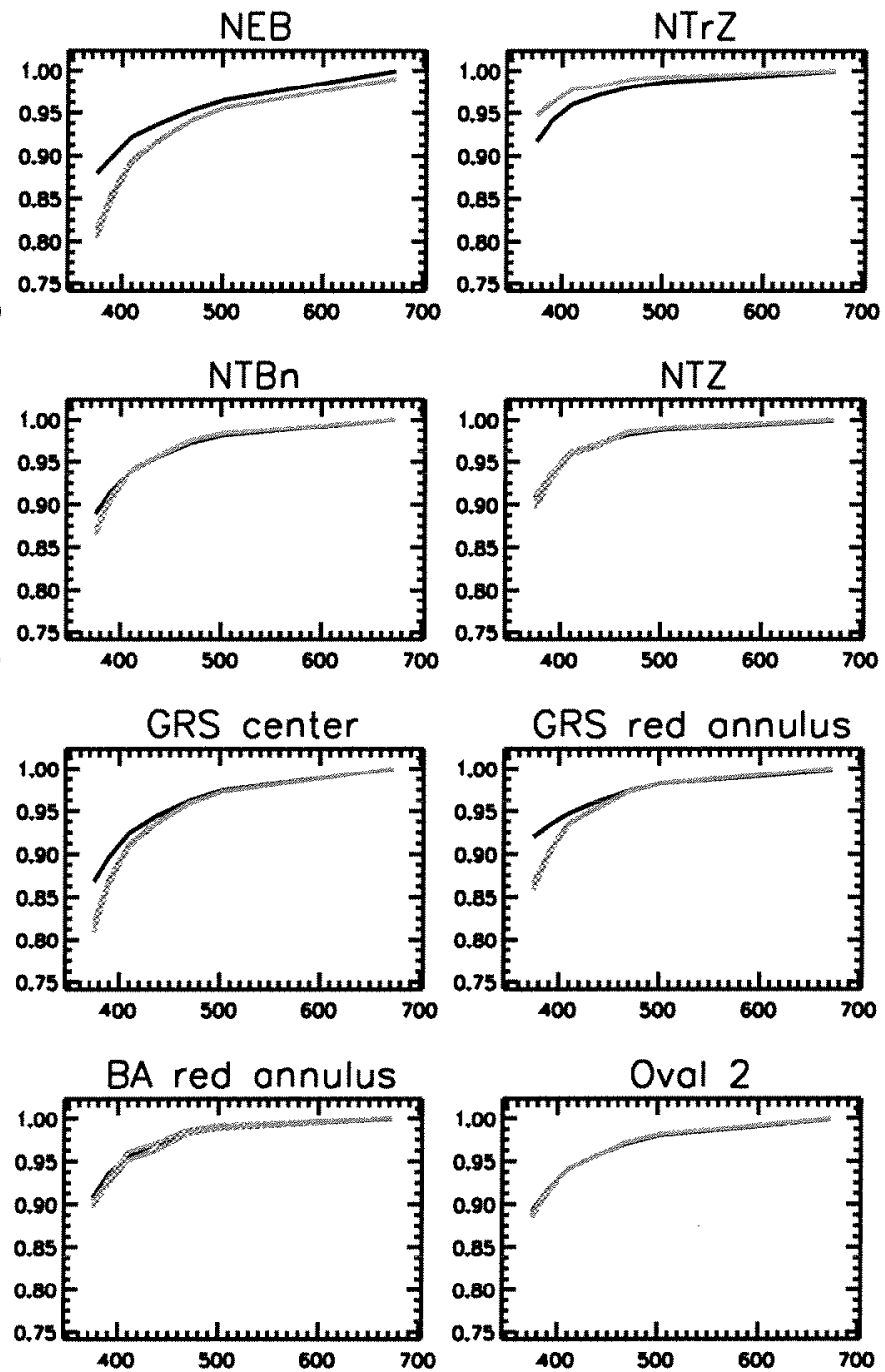

A5

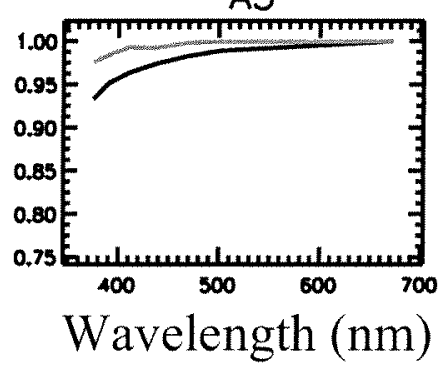

Fig. 18. The same as Fig. 12, except for retrievals from synthetic data with the radius of tropospheric cloud particles set to $4.0 \mu \mathrm{m}$. The retrievals assumed $2.0 \mu \mathrm{m}$ particles. 

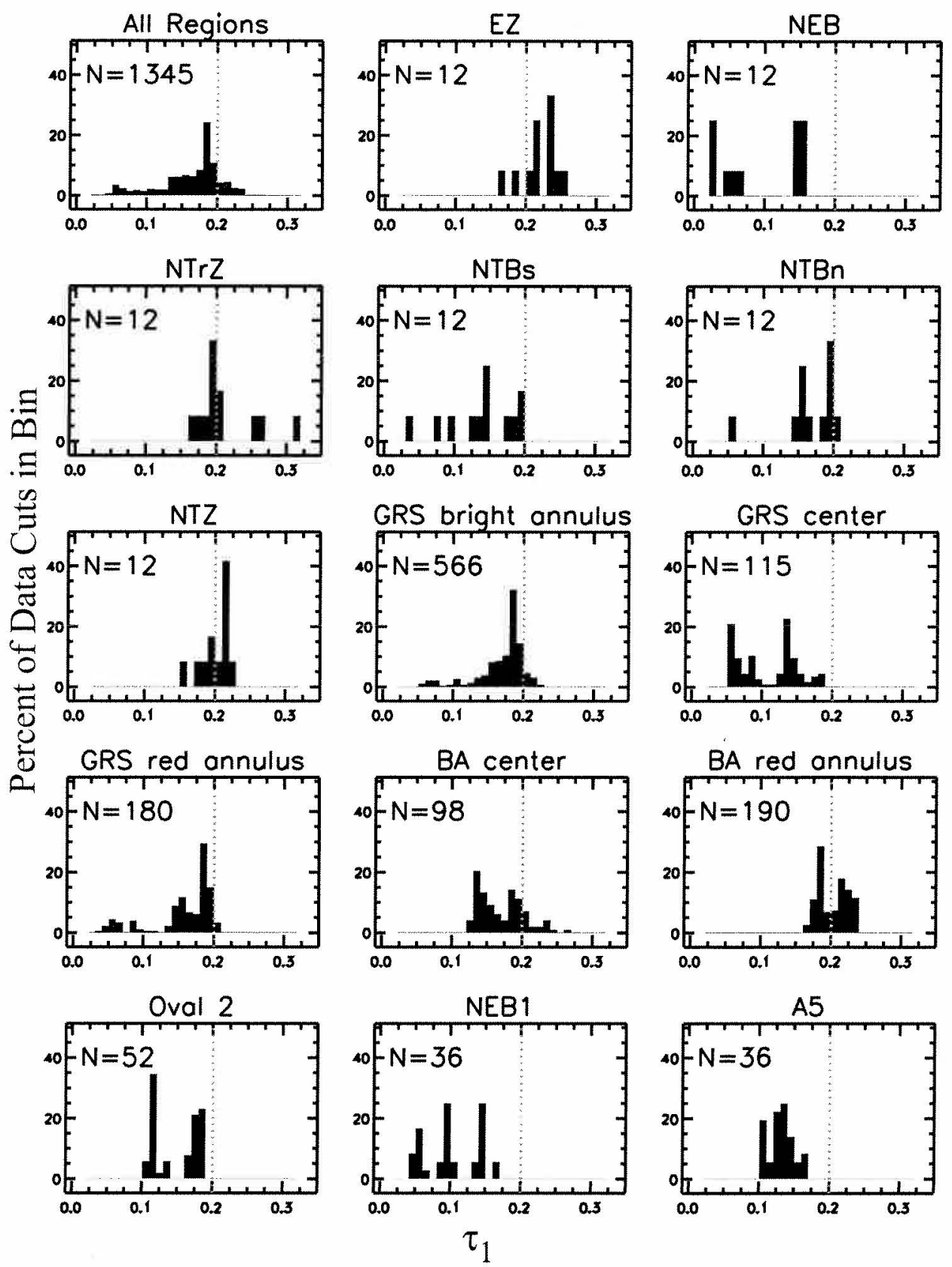

Fig. 19. The optical depth at $502 \mathrm{~nm}$ of the stratospheric haze $\left(\tau_{1}\right)$. All panels have the same abscissa and ordinate ranges. The dashed line is located at value of $\tau_{1}$ of the initial model provided to the $\chi^{2}$-minimization routine. The number $N$ denotes the number of data cuts modeled in that weather region. The bin size is 0.01 optical depths. 
All Regions

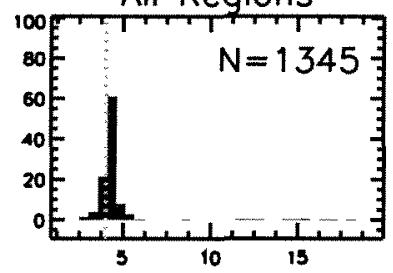

$N \operatorname{Tr} Z$

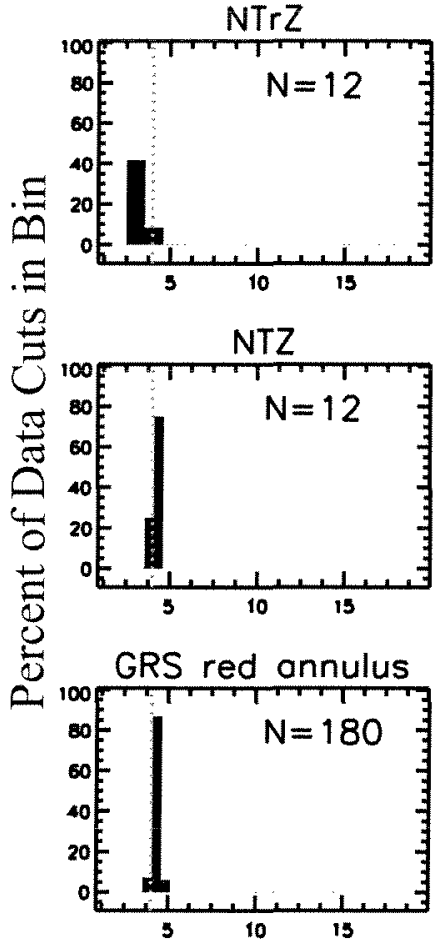

Oval 2

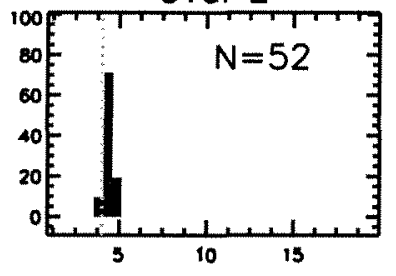

EZ

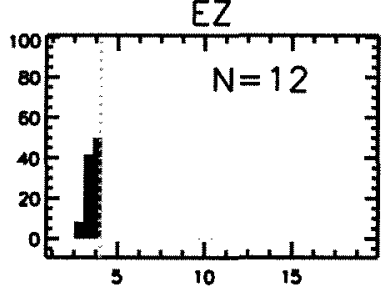

NTBs

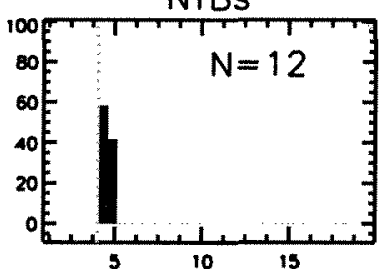

GRS bright annulus

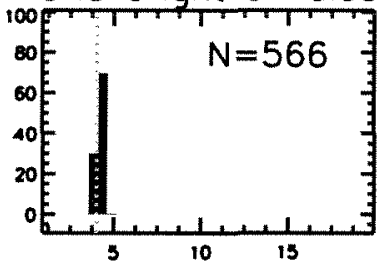

BA center

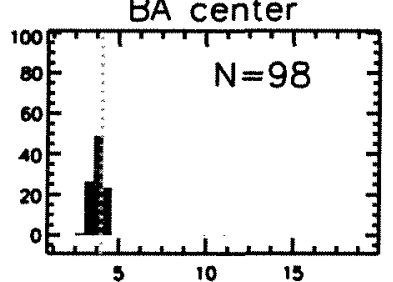

NEB 1

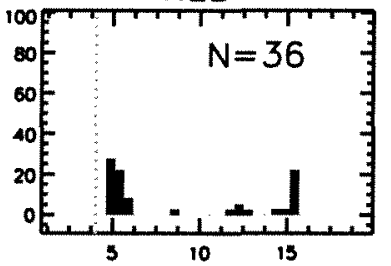

$\tau_{2}$
NEB

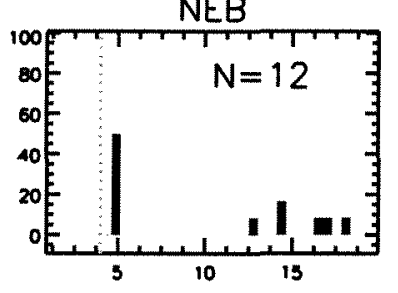

NTBn
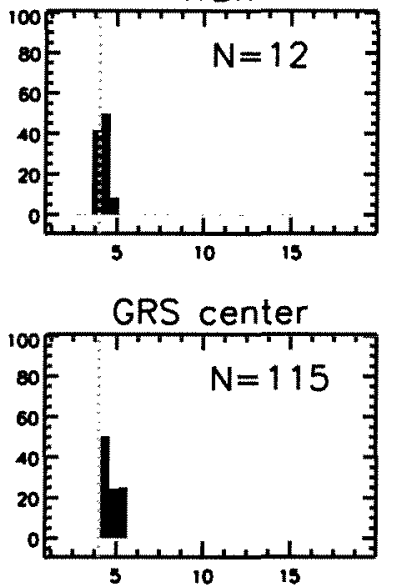

BA red annulus

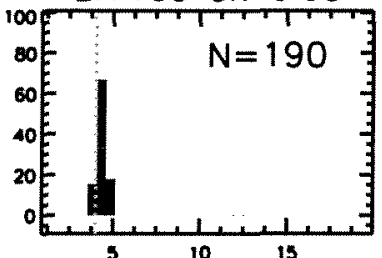

A5

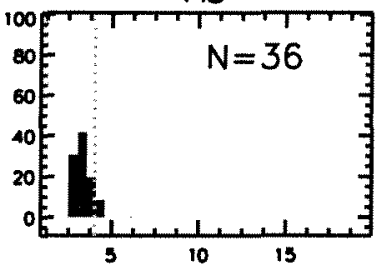

Fig. 20. The same as Fig. 19, except for the optical depth of the tropospheric haze $\left(\tau_{2}\right)$. The bin size is 0.5 optical depths. 
All Regions

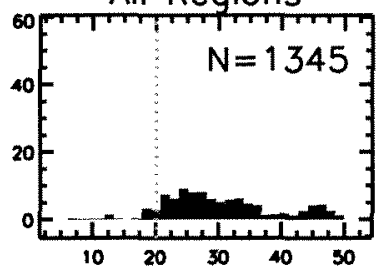

$N \operatorname{TrZ}$
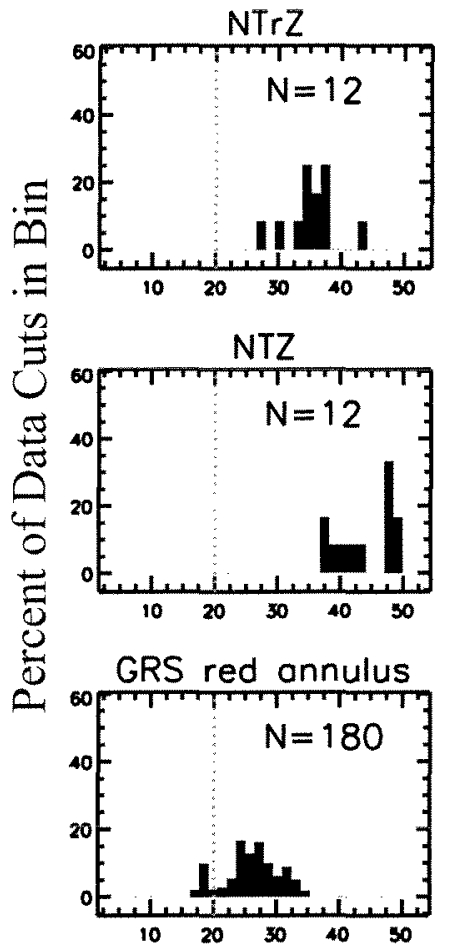

Oval 2

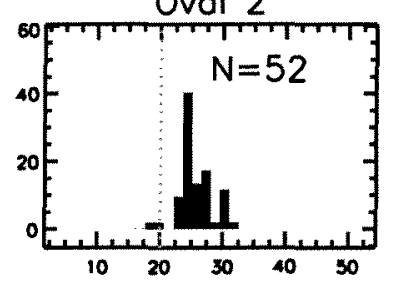

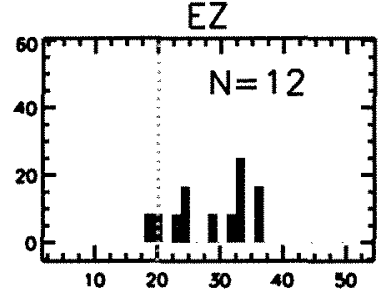
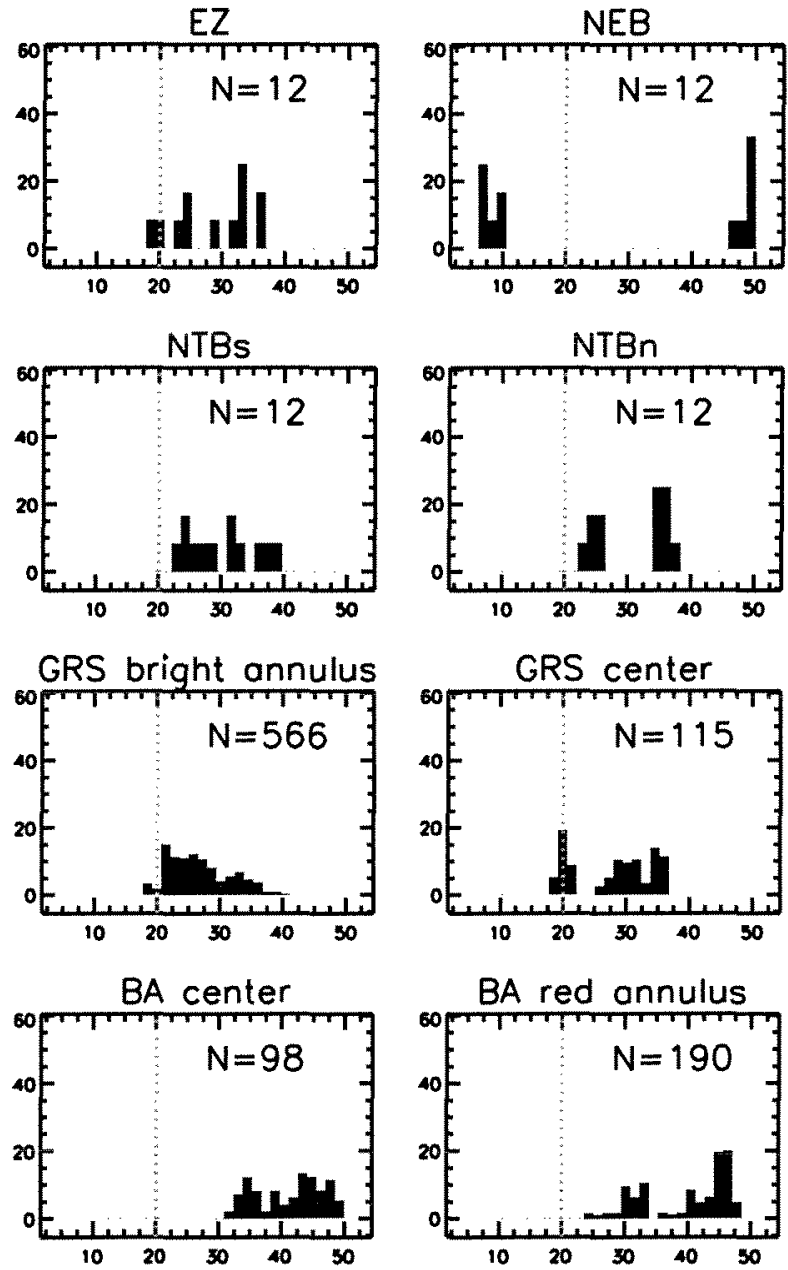

BA red annulus
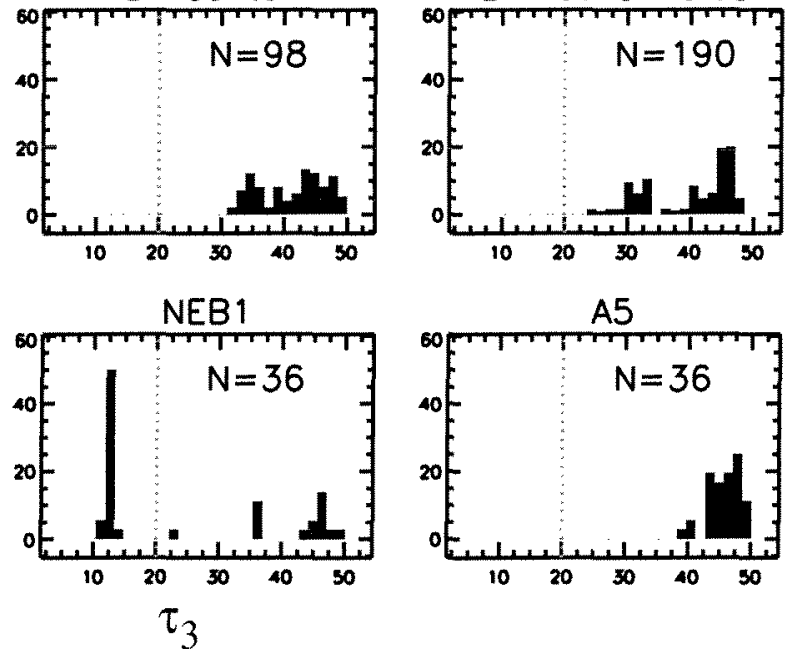

Fig. 21. The same as Fig. 19, except for the optical depth of the tropospheric cloud $\left(\tau_{3}\right)$. The bin size is 1.5 optical depths. 

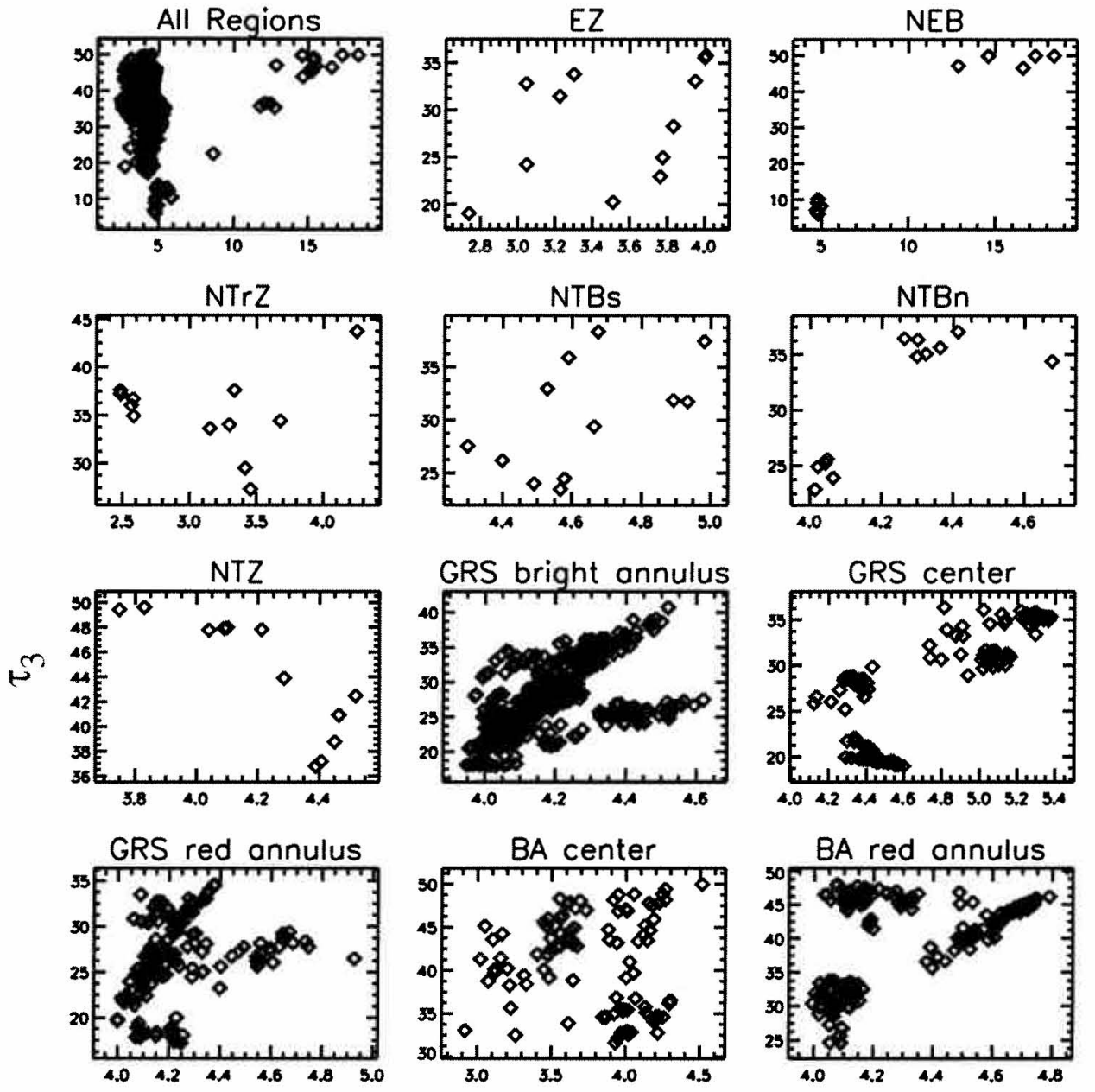

$B A$ red annulus
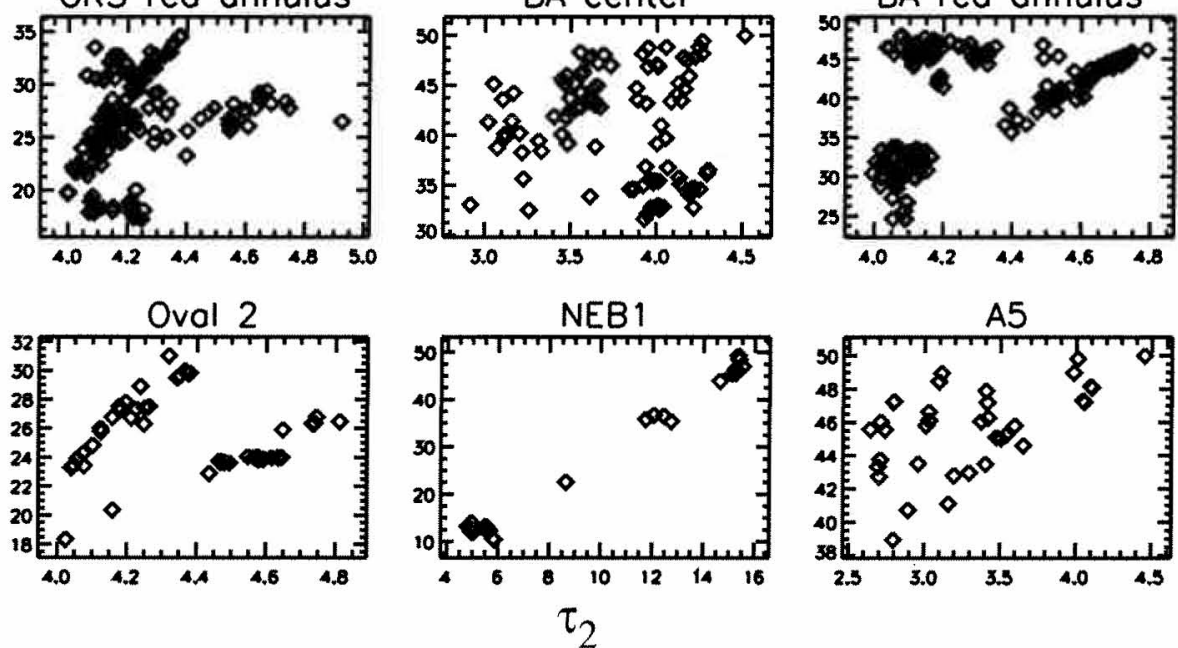

Fig. 22. The optical depth of the tropospheric haze $\left(\tau_{2}\right)$ versus cloud $\left(\tau_{3}\right)$. 


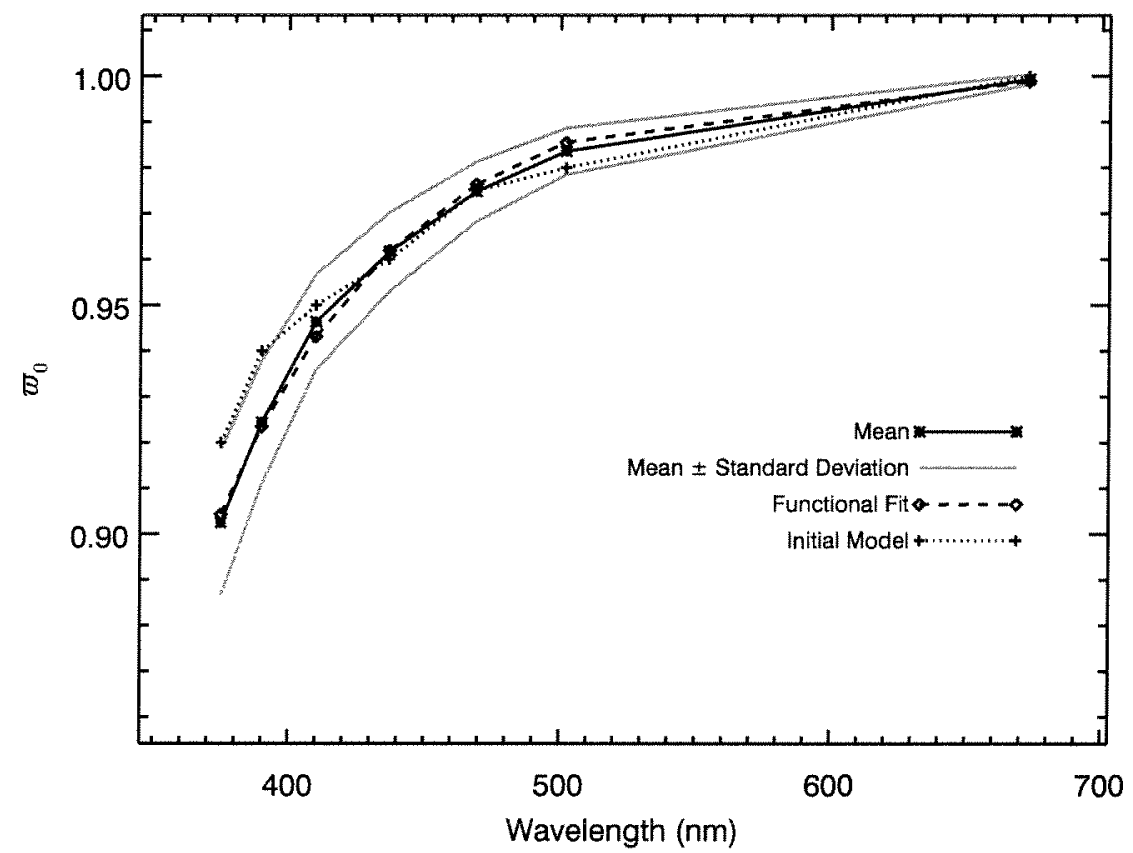

Fig. 23. The mean (solid black) and standard deviation (gray) of the retrieved single scattering albedo spectra $\left(\varpi_{0}(\lambda)\right)$ for all data cuts. The dashed black line is a functional fit to the mean with the form given in Eq. 7. The dotted black line is the initial model provided to the $\chi^{2}$ minimization routine. 

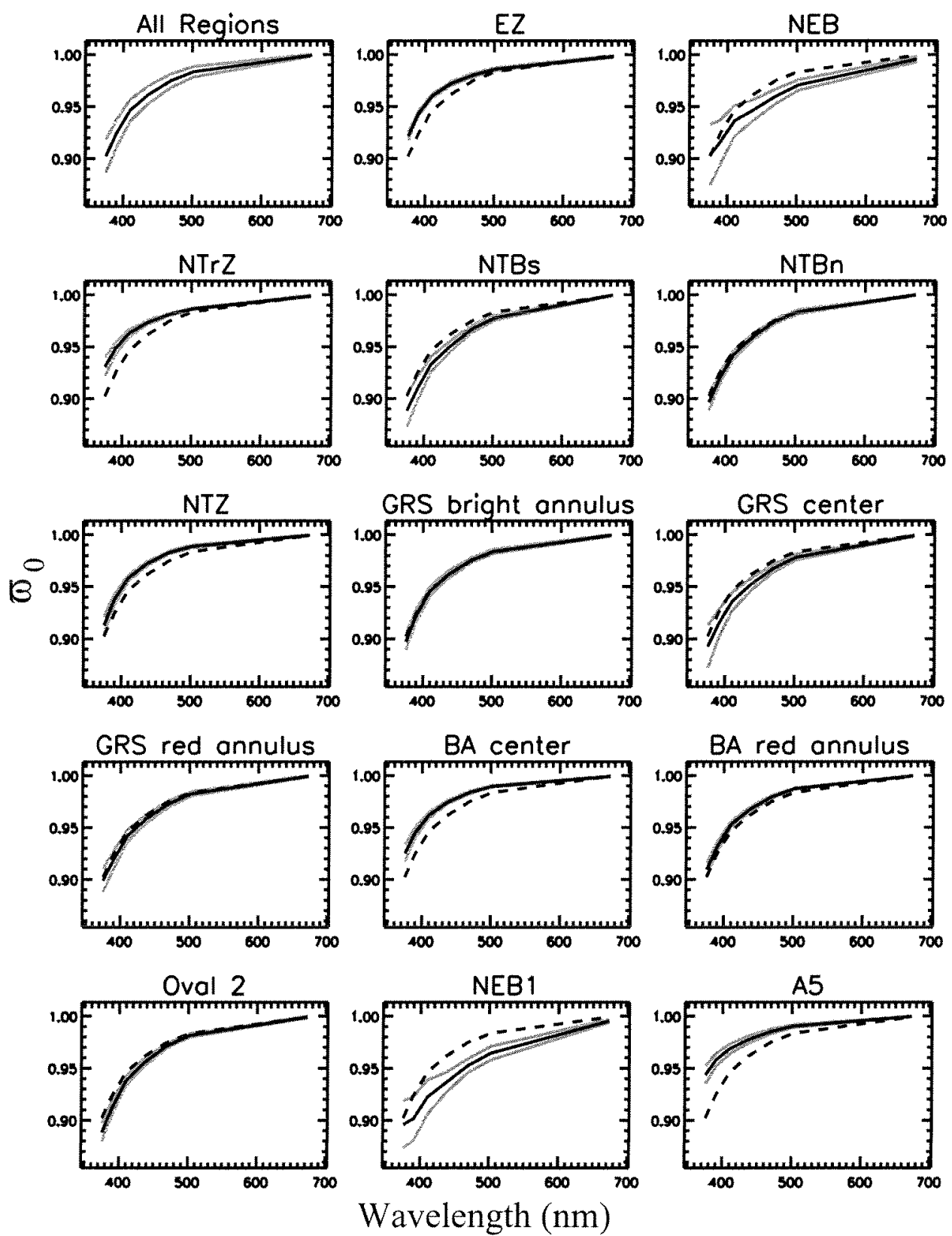

Fig. 24. The mean (solid black) and standard deviation (gray) of the retrieved $\varpi_{0}(\lambda)$ for all data cuts separated by weather region. The dashed black line is the mean for all data cuts from all weather regions and is repeated in each panel for ease of comparison between regions. All panels have the same abscissa and ordinate ranges. 

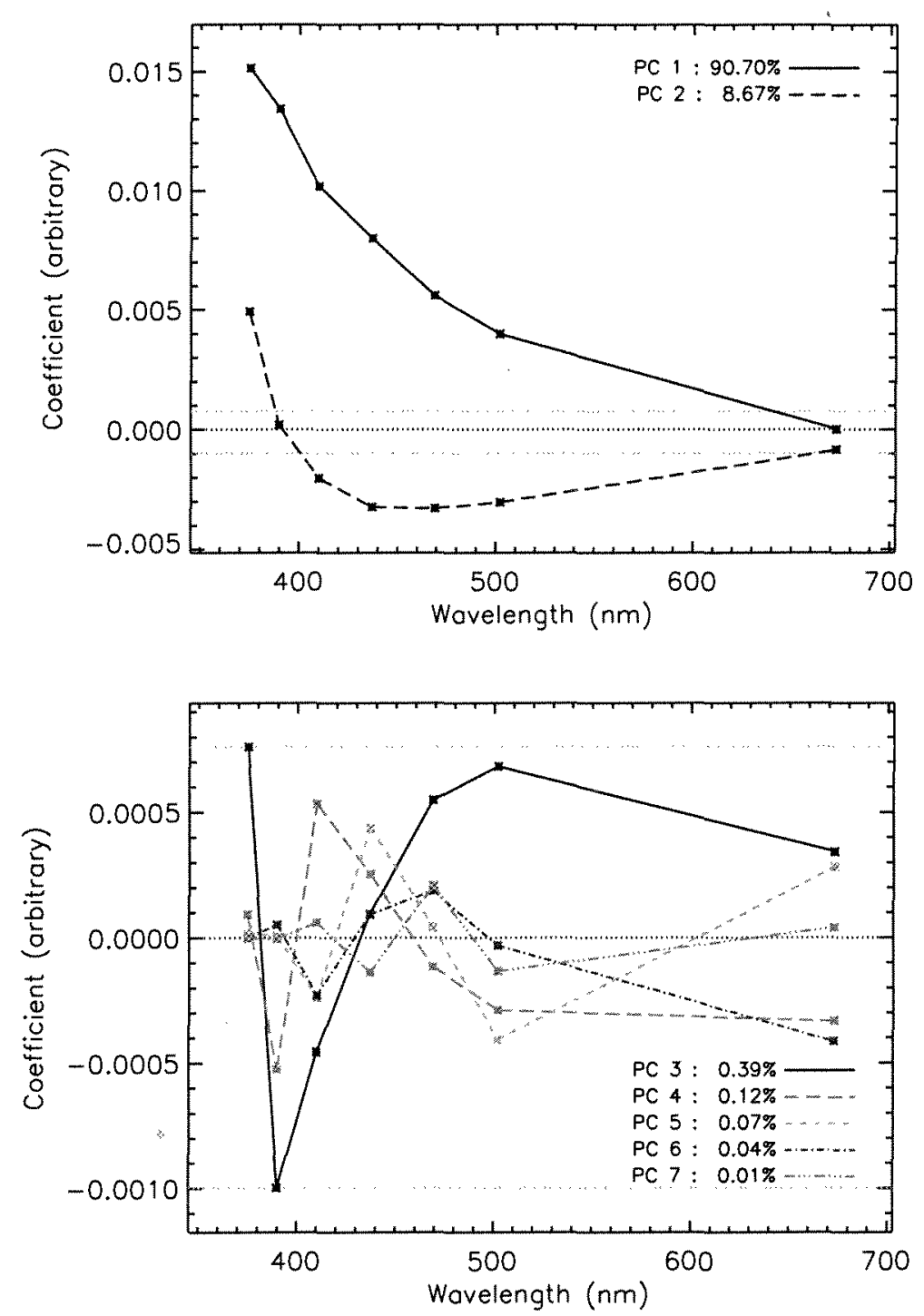

Fig. 25. Principal components (PCs) from a principal component analysis (PCA) of the derived single scattering albedo spectra for all data cuts. The number to the right of each $\mathrm{PC}$ denotes the percent of the total variance in the spectra described by that PC. The top panel shows PC1 and PC2. The bottom panel contains PCs 3-7 with a different ordinate scale. The gray dotted lines are at the same coefficient values in both panels for comparison between the two ordinate scales. The zero value is indicated with a black dotted line. For each PC, coefficients with the same sign are positively correlated, and coefficients with opposite signs are negatively correlated. 

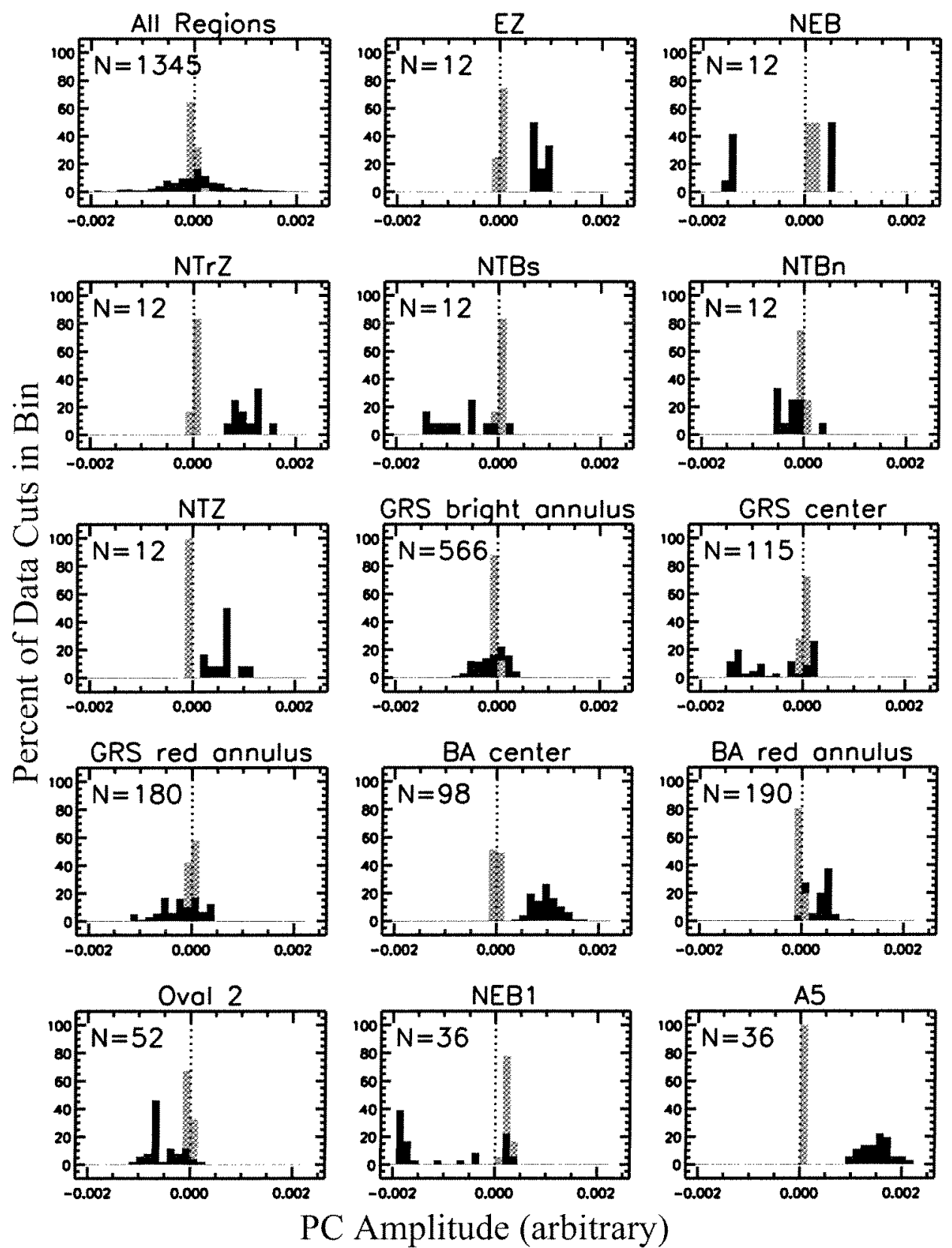

Fig. 26. Amplitudes of PC1 (black) and PC2 (gray) for each weather region. All panels have the same abscissa and ordinate ranges. The dashed line is located at an amplitude of zero. The number $N$ denotes the number of data cuts modeled in that weather region. The bin size is 0.0001 . 

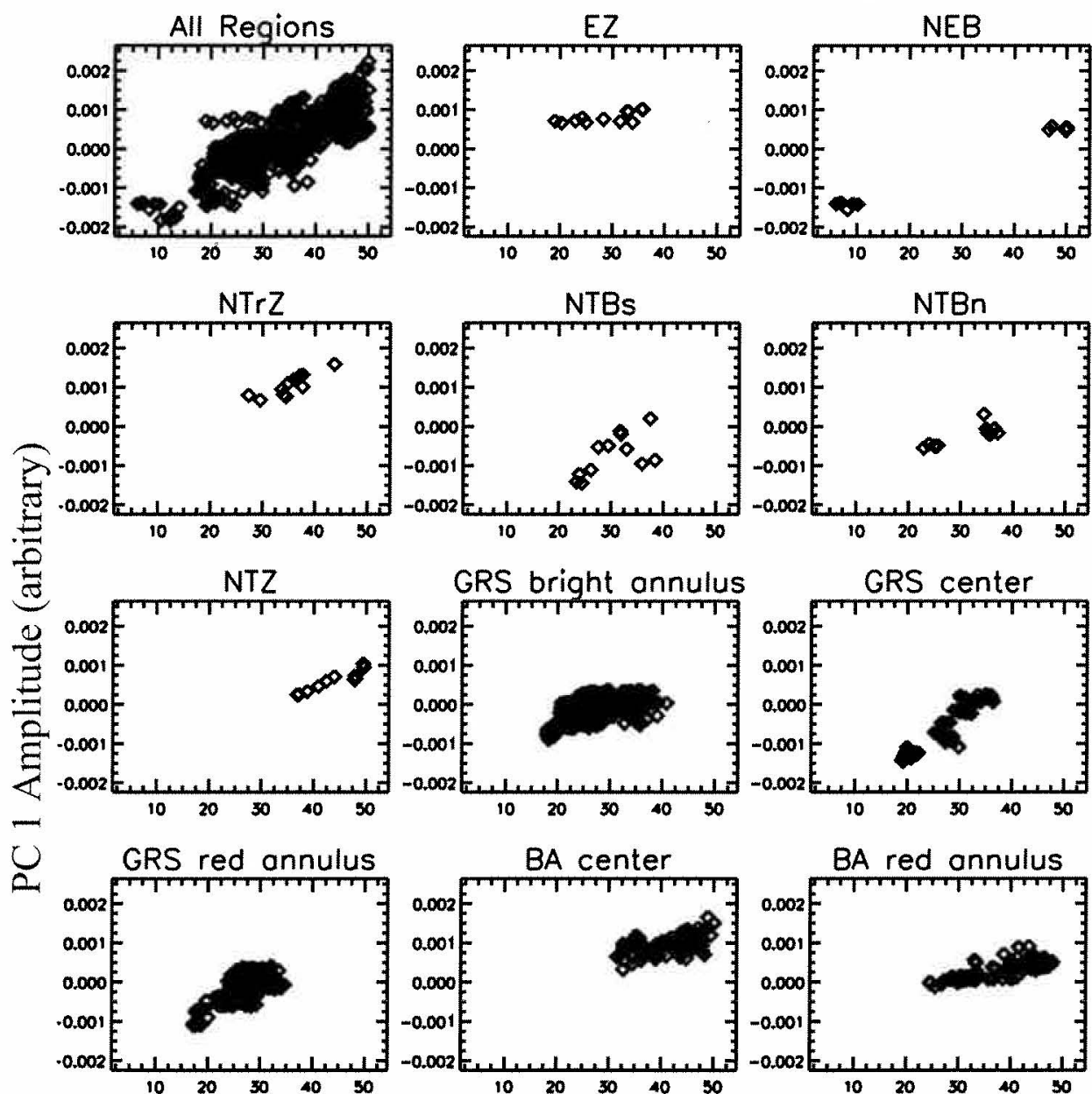

GRS bright annulus

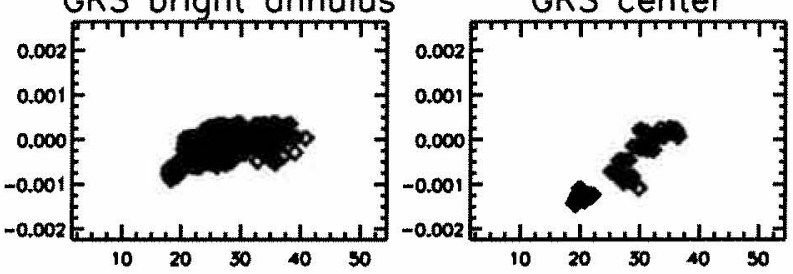

Oval 2

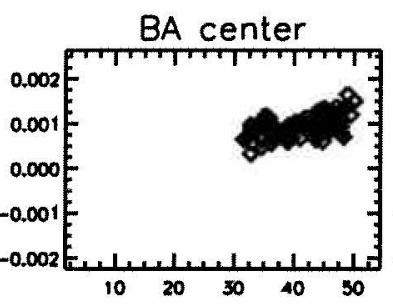

BA red annulus

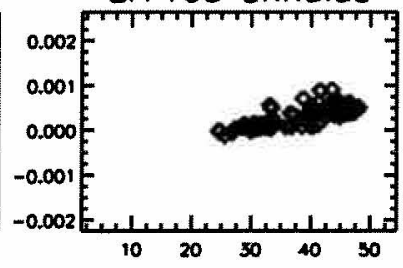

NEB 1
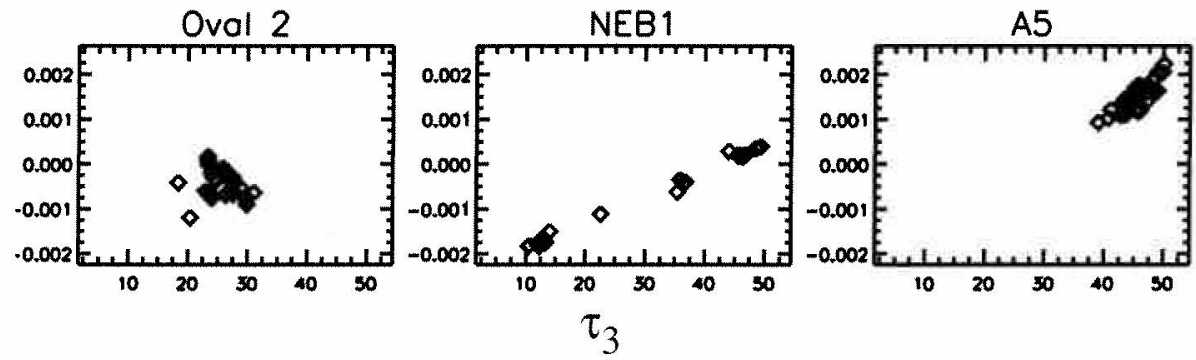

Fig. 27. The optical depth of the tropospheric cloud $\left(\tau_{3}\right)$ versus the amplitude of PC1 from the $\varpi_{0}(\lambda)$ PCA. All panels have the same abscissa and ordinate ranges. 

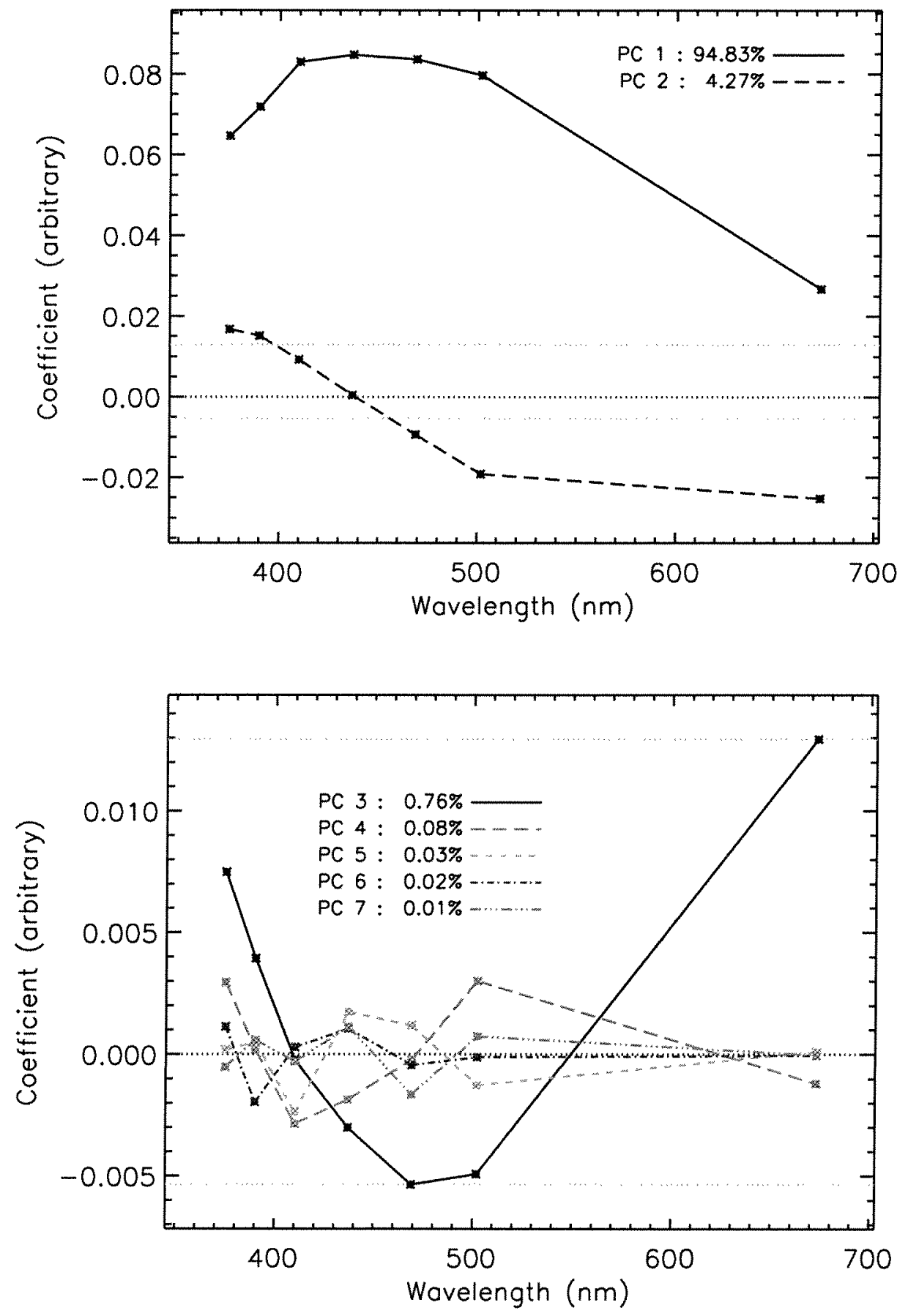

Fig. 28. The same as Fig. 25, except for a PCA of the $I / F_{0}(\lambda)$ for all data cuts. 

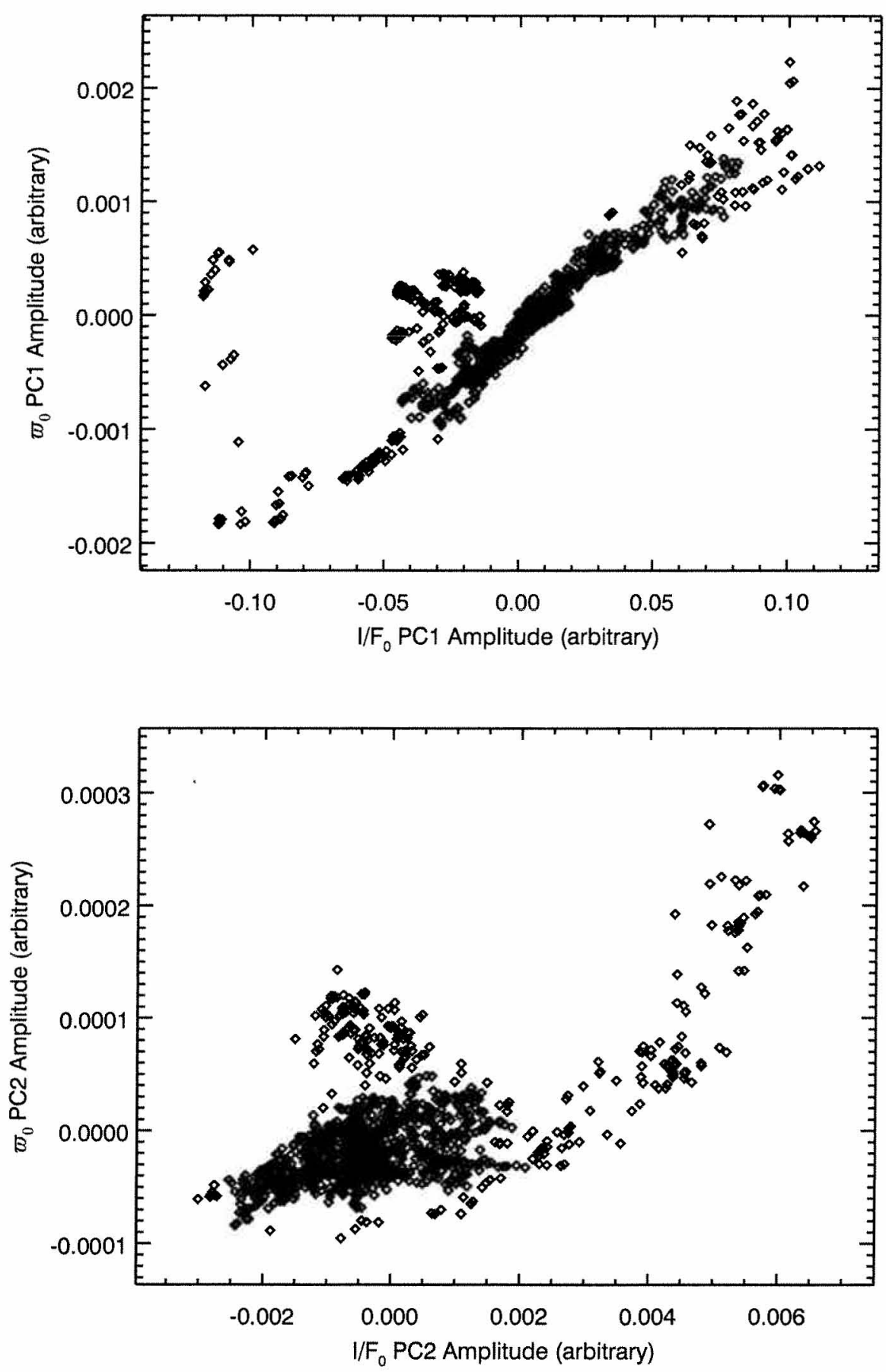

Fig. 29. Amplitudes of PCs from the $I / F_{0}(\lambda) \mathrm{PCA}$ are compared with those from the $\varpi_{0}(\lambda)$ PCA. The top panel shows the amplitudes of PC1, and the bottom panel shows the amplitudes of $\mathrm{PC} 2$. 